\title{
Quaternionic Quantum Field Theory
}

\author{
Stephen L. Adler \\ The Institute for Advanced Study, Princeton, NJ 08540, USA
}

\begin{abstract}
We show that a quaternionic quantum field theory can be formulated when the numbers of bosonic and fermionic degrees of freedom are equal and the fermions, as well as the bosons, obey a second order wave equation. The theory takes the form of either a functional integral with quaternion-imaginary Lagrangian, or a Schrödinger equation and transformation theory for quaternion-valued wave functions, with a quaternion-imaginary Hamiltonian. The connection between the two formulations is developed in detail, and many related issues, including the breakdown of the correspondence principle and the Hilbert space structure, are discussed.
\end{abstract}

\section{Introduction}

A basic theorem [1] in the foundations of quantum mechanics states that a general quantum mechanical system can be represented as a vector space with scalar coefficients drawn from the real, the complex, or the quaternion fields. ${ }^{1}$ Standard quantum mechanics and quantum field theory correspond to the complex case, while real quantum mechanics has been analyzed by Stueckelberg [3] and can be shown to reduce back to the complex case. Over the years a number of papers studying the quaternionic case have appeared and some useful mathematical and kinematical results have been obtained [4], but the central problem of finding a viable dynamics for quaternionic quantum theory has remained unsolved. We report progress on this problem in this paper. Specifically, we show ${ }^{2}$ that a dynamics for interacting quaternionic quantum fields can be formulated when the numbers of bosonic and fermionic degrees of freedom are equal and the fermions, as well as the bosons, obey a second-order wave equation.

\footnotetext{
${ }^{1}$ If the requirement of an associative multiplication is dropped, there is a fourth possibility, octonionic quantum mechanics, in which the scalar coefficients form a division algebra [2]. We assume an associative (but not commutative) multiplication in this paper, and so our analysis does not apply to the octonionic case

${ }^{2}$ A brief, partial account of the results of this paper appeared in [5]
} 
We begin with a few general remarks. An element $\phi$ of the quaternion field has the form

$$
\phi=\phi_{0}+\phi_{1} e_{1}+\phi_{2} e_{2}+\phi_{3} e_{3},
$$

with $e_{1,2,3}$ the quaternion units satisfying the algebra

$$
e_{a} e_{b}=-\delta_{a b}+\varepsilon_{a b c} e_{c}, \quad a, b, c=1,2,3,
$$

and with $\phi_{0,1,2,3}$ real numbers. The conjugate quaternion $\bar{\phi}$ is defined by

$$
\bar{\phi}=\phi_{0}-\phi_{1} e_{1}-\phi_{2} e_{2}-\phi_{3} e_{3},
$$

and so quaternionic quantum theory has three imaginary units instead of the single imaginary unit $e_{3}$ or $i$ of complex quantum theory, and these imaginary units do not commute. The differences between quaternionic and complex quantum theory all stem from these facts. For example, since there are three imaginary units we cannot automatically convert an anti-Hermitian operator to a Hermitian one by multiplying by an imaginary unit.

Since we expect only a subset of the usual formalism of complex quantum theory to generalize to the quaternionic case, we must step back and ask which features of the standard formalism should be part of any generalized quantum mechanics, and which are expendable? We will adopt in this paper the philosophy that a generalized quantum mechanical system is one which satisfies the principle of superposition of probability amplitudes. We will show that when there is complete boson-fermion symmetry, this starting point can be naturally developed by functional integration methods into a quaternionic dynamics and a quaternionic transformation theory. Our emphasis will be exclusively on the dynamics of time development; hence spatial coordinates will never explicitly appear in this paper, but rather are subsumed in a discrete field index.

The organization of this paper is as follows. In Sect. 2 we summarize the spectral properties of quaternionic matrices, outline the theory of quaternionic Gaussian multiple integrals [6], and derive a formula for the quaternionic delta function. In Sect. 3 we formulate a sum-over-histories (functional integration) approach to quaternionic quantum field theory in terms of a quaternionimaginary Lagrangian. In Sect. 4 we derive the Schrödinger equation from the functional integral and thus identify the quaternion-imaginary Hamiltonian. A quaternionic transformation theory is developed in Sect. 5 and is used to rederive the functional integral from the Schrödinger equation. In Sect. 6 we show that the quaternionic field dynamics can be projected down to a complex field dynamics (still with second-order fermion wave operator) when the interactions are not intrinsically quaternionic. The correspondence principle, which relates quantum to classical field equations, is shown in Sect. 7 to break down in quaternionic quantum mechanics. In Sects. 8 and 9 we examine the state structure which is implicit in the preceding derivations; the boson states are discussed in Sect. 8 and the fermion states in Sect. 9. We show that the fermion states require an indefinite metric Hilbert space, and that a first-order wave operator is probably not permitted in quaternionic field theory. Finally, in Sect. 10 we discuss open issues and future directions, including the possible relevence of quaternionic quantum field theory to elementary particle physics. 


\section{Kinematic Preliminaries: Quaternionic Variables, Quaternionic Matrices and their Spectral Properties, Quaternionic Gaussian Multiple Integrals, and the Quaternionic Delta Function}

We give in this section some kinematic preliminaries which are needed later on. Throughout this paper we will deal with two kinds of quaternionic variables: (i) Real or bosonic quaternions $\phi, \bar{\phi}$, as in Eqs. (1.1)-(1.3), with components $\phi_{a}$, $a=0,1,2,3$ which are real numbers, and (ii) Grassmann or fermionic quaternions $\psi, \bar{\psi}$,

$$
\psi=\psi_{0}+\psi_{1} e_{1}+\psi_{2} e_{2}+\psi_{3} e_{3}, \quad \bar{\psi}=\psi_{0}-\psi_{1} e_{1}-\psi_{2} e_{2}-\psi_{3} e_{3},
$$

with components $\psi_{a}$ which are real Grassmann numbers. We adopt the convention (justified in Sect. 9 below) that the product of any number of real numbers and any number of real Grassmann elements is real. Then from Eqs. (1.1)-(1.3) and (2.1) we find that if $\phi, \phi^{\prime}$ are two bosonic quaternions and $\psi, \psi^{\prime}$ are two Grassmann quaternions, the conjugates of their products are given by

$$
\begin{aligned}
& \overline{\phi \phi^{\prime}}=\overline{\phi^{\prime}} \bar{\phi}, \\
& \overline{\psi \psi^{\prime}}=-\overline{\psi^{\prime}} \bar{\psi}, \\
& \overline{\phi \psi}=\bar{\psi} \bar{\phi},
\end{aligned}
$$

with the - sign in Eq. (2.2b) arising from anticommutation of the Grassmann elements.

An $N \times N$ quaternion matrix $M$ has matrix elements $M_{i j}, i, j=1, \ldots, N$ which are real quaternions. The conjugate matrix $\bar{M}$ has matrix elements $\bar{M}_{i j}$, while the adjoint matrix $M^{\dagger}$ has matrix elements

$$
M_{i j}^{\dagger}=\bar{M}_{j i}
$$

together with Eq. (2.2) this definition implies that any two quaternion matrices $M, N$ obey

$$
(M N)^{\dagger}=N^{\dagger} M^{\dagger} \text {. }
$$

A quaternion matrix $M$ is called self-adjoint if

$$
M=M^{\dagger},
$$

anti-self-adjoint if

$$
M=-M^{\dagger},
$$

and these properties are preserved under a general quaternion unitary transformation

$$
M \rightarrow U^{\dagger} M U, \quad U U^{\dagger}=U^{\dagger} U=1 .
$$

Self-adjoint and anti-self-adjoint quaternion matrices have special spectral properties. For any quaternion self-adjoint matrix $M$, one can find [7] a unitary $U$ such that $U^{\dagger} M U=D$ with $D$ diagonal and real,

$$
D_{i j}=d_{i} \delta_{i j}, \quad d_{i}=\bar{d}_{i} .
$$


For any quaternion anti-self-adjoint matrix $M$, one can find ${ }^{3}$ a unitary $U$ such that $U^{\dagger} M U=D e_{3}$, with $D$ diagonal, real and positive,

$$
D_{i j}=d_{i} \delta_{i j}, \quad d_{i}=\bar{d}_{i} \geqq 0 .
$$

(The reason that the $d_{i}$ can be assumed to be positive is that $\bar{e}_{2} e_{3} e_{2}=-e_{3}$, permitting any diagonal factors of -1 to be absorbed in the definition of $U$.) For $M$ self-adjoint, a quaternion determinant $\operatorname{det} M$ can be defined (see Dyson and Mehta [4]) which has the product decomposition

$$
\operatorname{det} M=\prod_{i=1}^{N} d_{i}
$$

with $d_{i}$ the eigenvalues of Eq. (2.7). For $M$ anti-self-adjoint, the determinant of $M^{\dagger} M$ has the analogous product decomposition

$$
\operatorname{det}\left(M^{\dagger} M\right)=\prod_{i=1}^{N} d_{i}^{2},
$$

with $d_{i}$ the eigenvalues of Eq. (2.8).

We are now ready to state the quaternionic Gaussian integration formula [6] which plays a fundamental role in our construction of quaternionic quantum field theory,

$$
\begin{aligned}
\lim _{\varepsilon \rightarrow 0}( & \left.\prod_{i=1}^{N} \int d \phi^{i}\right)\left(\prod_{i=1}^{M} \int d \psi^{i}\right)\left(4 \pi^{2}\right)^{-N} \\
& \cdot \exp (-\bar{\phi} A \phi-\bar{\psi} B \psi+\bar{u} \phi-\bar{\phi} u+\bar{\xi} \psi+\bar{\psi} \xi+\mathfrak{R}-\varepsilon \bar{\phi} \phi) \\
= & 4^{M-N} \operatorname{det}^{2} B \operatorname{det}^{-1}\left(A^{\dagger} A\right) L_{N-M}\left(-\bar{u} A^{-1} u+\bar{\xi} B^{-1} \xi+\Re\right),
\end{aligned}
$$

with $L_{I}$ the function defined by

$$
\begin{gathered}
L_{I}(\mathbf{j} \cdot \mathbf{e})=c_{I}(j)+\frac{\mathbf{j} \cdot \mathbf{e}}{j} d_{I}(j), \quad j=|\mathbf{j}|, \quad c_{I}(j)=\cos j-\frac{2 I}{j} \sin j, \\
d_{I}(j)=-\frac{d}{d j} c_{I}(j)=\left(1-\frac{2 I}{j^{2}}\right) \sin j+\frac{2 I}{j} \cos j .
\end{gathered}
$$

Our notation is as follows: $\phi$ and $u$ are column vectors containing $N$ real quaternions, and $\psi$ and $\xi$ are column vectors containing $M$ real Grassmann quaternions, $A=-A^{\dagger}$ is an $N \times N$ quaternion anti-self-adjoint matrix, $B=B^{\dagger}$ is an $M \times M$ quaternion self-adjoint matrix, $\Re=-\bar{\Re}$ is a fixed imaginary quaternion, and the integration measure is defined by ${ }^{4}$

$$
d \phi^{i}=d \phi_{0}^{i} d \phi_{1}^{i} d \phi_{2}^{i} d \phi_{3}^{i}, \quad d \psi^{i}=d \psi_{0}^{i} d \psi_{1}^{i} d \psi_{2}^{i} d \psi_{3}^{i} .
$$

The fact that the argument of $L_{I}$ is quaternion imaginary follows from Eqs. (2.2)-(2.4), and similarly the argument of the exponential in the integrand of

\footnotetext{
${ }^{3}$ The proof of the spectral theorem for anti-self-adjoint matrices is a close analog of the proof of the spectral theorem for quaternion unitary operators given by Finkelstein, Jauch, and Speiser [7] ${ }^{4}$ A supermatrix generalization of Eq. (2.11) is also given in [6], but is not needed for the derivations which follow. The definitions of Eq. (2.13) differ by numerical factors from those of [6]
} 
Eq. (2.11) is quaternion imaginary, apart from the infinitesimal convergence factor $\exp (-\varepsilon \bar{\phi} \phi)$. Introducing the abbreviation $\mathfrak{I}=\mathbf{j} \cdot \mathbf{e}$, the formula for $L_{I}$ in Eq. (2.12) can be rewritten as the power series

$$
L_{I}(\mathfrak{I})=\sum_{n=0}^{\infty}\left\{\mathfrak{J}^{2 n}\left[\frac{1}{(2 n) !}-\frac{2 I}{(2 n+1) !}\right]+\mathfrak{J}^{2 n+1}\left[\frac{1}{(2 n+1) !}-\frac{4 I(n+1)}{(2 n+3) !}\right]\right\} .
$$

When $I=0$, Eqs. (2.12) and (2.14) show that the function $L$ reduces to an exponential,

$$
L_{0}(\mathfrak{J})=\exp \mathfrak{I},
$$

and hence when the number of bosonic degrees of freedom $N$ is equal to the number of fermionic degrees of freedom $M$, Eq. (2.11) simplifies to

$$
\begin{aligned}
\lim _{\varepsilon \rightarrow 0} & \left(\prod_{i=1}^{M} \int d \phi^{i} d \psi^{i}\right)\left(4 \pi^{2}\right)^{-M} \\
& \cdot \exp (-\bar{\phi} A \phi-\bar{\psi} B \psi+\bar{u}-\bar{\phi} u+\bar{\xi} \psi+\bar{\psi} \xi+\Omega-\varepsilon \bar{\phi} \phi) \\
= & \operatorname{det}^{2} B \operatorname{det}^{-1}\left(A^{\dagger} A\right) \exp \left(-\bar{u} A^{-1} u+\bar{\xi} B^{-1} \xi+\Re\right) .
\end{aligned}
$$

Equation (2.16) is the starting point for the analysis of the sections which follow.

We briefly sketch the derivation of Eqs. (2.11)-(2.12), since the methods used will be needed in the Schrödinger equation derivation of Sect. 4 . We begin by making the change of integration variables

$$
\phi \rightarrow U_{1} \phi-A^{-1} u, \quad \bar{\phi} \rightarrow \bar{\phi} U_{1}^{\dagger}+\bar{u} A^{-1}, \quad \psi \rightarrow U_{2} \psi+B^{-1} \xi, \quad \bar{\psi} \rightarrow \bar{\psi} U_{2}^{\dagger}+\bar{\xi} B^{-1},
$$

with $U_{1,2}$ the quaternion unitary matrices which diagonalize $A$ and $B$ respectively. Since the substitution of Eq. (2.17) can be shown [6] to be an invariance of the integration measure in Eq. (2.11), the integral reduces to

$$
\begin{gathered}
\lim _{\varepsilon \rightarrow 0}\left(\prod_{i=1}^{N} \int d \phi^{i}\right)\left(\prod_{i=1}^{M} \int d \psi^{i}\right)\left(4 \pi^{2}\right)^{-N} \exp \left(-\bar{\phi} e_{3} D_{A} \phi-\bar{\psi} B_{B} \psi+\mathfrak{I}-\varepsilon \bar{\phi} \phi\right), \\
\mathfrak{J}=-\bar{u} A^{-1} u+\bar{\xi} B^{-1} \xi+\mathfrak{K},
\end{gathered}
$$

with $e_{3} D_{A}$ and $D_{B}$ the diagonal forms of $A$ and $B$. Making the rescalings

$$
\phi_{a}^{i} \rightarrow\left(D_{A}^{i}\right)^{-1 / 2} \phi_{a}^{i},
$$

and noting that since $\bar{\psi}^{i} D_{B}^{i} \psi^{i}$ is quadratic in the Grassmann components of $\psi^{i}$, the only contributions to Eq. (2.18) come from terms in the power series expansion of the exponential which contain exactly two factors $\bar{\psi}^{i} D_{B}^{i} \psi^{i}$ for each $i$, Eq. (2.18) reduces further to

$$
\begin{gathered}
\operatorname{det}^{2} B \operatorname{det}^{-1}\left(A^{\dagger} A\right) I_{N, M}(\mathfrak{I}), \\
I_{N, M}(\mathfrak{I})=\lim _{\varepsilon \rightarrow 0}\left[\prod_{i=1}^{N} \int d \phi^{i} \exp \left(-\varepsilon \overline{\phi^{i}} \phi^{i}\right)\right]\left(\prod_{i=1}^{M} \int d \psi^{i}\right)\left(4 \pi^{2}\right)^{-N} \\
\cdot \exp \left[-\sum_{i=1}^{N} \overline{\phi^{i}} e_{3} \phi^{i}-\sum_{i=1}^{M} \bar{\psi}^{i} \psi^{i}+\mathfrak{J}\right] .
\end{gathered}
$$


To calculate $I_{N, M}$, we write

$$
\mathfrak{I}=\mathbf{j} \cdot \mathbf{e}, \quad \sum_{i=1}^{N} \bar{\phi}^{i} e_{3} \phi^{i}-\sum_{i=1}^{M} \bar{\psi}^{i} \psi^{i}=\mathbf{v} \cdot \mathbf{e}
$$

and rewrite the exponential in Eq. (2.20) as

$$
\exp [(\mathbf{j}+\mathbf{v}) \cdot \mathbf{e}]=\cos |\mathbf{j}+\mathbf{v}|+\frac{(\mathbf{j}+\mathbf{v}) \cdot \mathbf{e}}{|\mathbf{j}+\mathbf{v}|} \sin |\mathbf{j}+\mathbf{v}| .
$$

Since $I_{N, M}$ must have the general form

$$
I_{N, M}(\mathfrak{J})=I_{N, M}^{R}(j)+\frac{\mathbf{j} \cdot \mathbf{e}}{j} I_{N, M}^{I}(j), \quad j=|\mathbf{j}|,
$$

we can project out separate formulas for $I_{N, M}^{R, I}(j)$, giving after a little algebra

$$
\begin{gathered}
I_{N, M}^{I}(j)=-\frac{d}{d j} I_{N, M}^{R}(j) \\
I_{N, M}^{R}(j)=\lim _{\varepsilon \rightarrow 0}\left[\prod_{i=1}^{N} \int d \phi^{i} \exp \left(-\varepsilon \bar{\phi}^{i} \phi^{i}\right)\right]\left(\prod_{i=1}^{M} \int d \psi^{i}\right)\left(4 \pi^{2}\right)^{-N} \cos |\mathbf{v}+\mathbf{j}| .
\end{gathered}
$$

Hence it suffices to evaluate $I_{N, M}^{R}(j)$. Writing

$$
\begin{array}{cc}
\bar{\phi}^{i} e_{3} \phi^{i}=\mathbf{s}\left(\phi^{i}\right) \cdot \mathbf{e}, & \bar{\psi}^{i} \psi^{i}=-\mathbf{r}\left(\psi^{i}\right) \cdot \mathbf{e}, \\
s_{1}(\phi)=2\left(\phi_{3} \phi_{1}-\phi_{0} \phi_{2}\right), & r_{1}(\psi)=2\left(\psi_{2} \psi_{3}-\psi_{0} \psi_{1}\right), \\
s_{2}(\phi)=2\left(\phi_{2} \phi_{3}+\phi_{0} \phi_{1}\right), & r_{2}(\psi)=2\left(\psi_{3} \psi_{1}-\psi_{0} \psi_{2}\right), \\
s_{3}(\phi)=\phi_{0}^{2}+\phi_{3}^{2}-\phi_{1}^{2}-\phi_{2}^{2}, & r_{3}(\psi)=2\left(\psi_{1} \psi_{2}-\psi_{0} \psi_{3}\right),
\end{array}
$$

we can reexpress Eq. (2.24b) as recursion relations describing the effect of adding an additional bosonic or fermionic integral to $I_{N, M}^{R}$,

$$
\begin{gathered}
I_{N, M}^{R}(j)=\lim _{\varepsilon \rightarrow 0} \int d \phi \exp (-\varepsilon \bar{\phi} \phi)\left(4 \pi^{2}\right)^{-1} I_{N-1, M}^{R}(|\mathbf{j}-\mathbf{s}(\phi)|), \\
I_{N, M}^{R}(j)=\int d \psi I_{N, M-1}^{R}(|\mathbf{j}+\mathbf{r}(\psi)|) .
\end{gathered}
$$

To simplify the bosonic recursion relation of Eq. (2.26a), we introduce polar coordinates for the quaternionic integration variable $\phi$ as follows,

$$
\begin{gathered}
\phi_{0}=R \cos \theta_{1} \cos \Theta / 2, \quad \phi_{3}=R \sin \theta_{1} \cos \Theta / 2, \\
\phi_{1}=R \cos \theta_{2} \sin \Theta / 2, \quad \phi_{2}=R \sin \theta_{2} \sin \Theta / 2, \\
\int d \phi_{0} d \phi_{1} d \phi_{2} d \phi_{3}=\frac{1}{4} \int_{0}^{\infty} R^{3} d R \int_{0}^{\pi} \sin \Theta d \Theta \int_{0}^{2 \pi} d \theta_{1} \int_{0}^{2 \pi} d \theta_{2} .
\end{gathered}
$$

The utility of this parameterization becomes clear when we compute $\bar{\phi} \phi$ and $\mathbf{s}(\phi)$,

$$
\begin{gathered}
\bar{\phi} \phi=R^{2}, \quad s_{1}(\phi)=R^{2} \sin \Theta \sin \left(\theta_{1}-\theta_{2}\right), \\
s_{2}(\phi)=R^{2} \sin \Theta \cos \left(\theta_{1}-\theta_{2}\right), \quad s_{3}(\phi)=R^{2} \cos \Theta ;
\end{gathered}
$$


evidently the angles $\Theta, \theta_{1}-\theta_{2}$ are just the polar coordinates of the vector $\mathbf{s}$. Writing $s=R^{2}$ and denoting the angular measure for $\hat{s}$ by $d \Omega_{\hat{s}}$, Eq. (2.26a) takes the form

$$
I_{N, M}^{R}(j)=\lim _{\varepsilon \rightarrow 0}(16 \pi)^{-1} \int_{0}^{\infty} s d s e^{-\varepsilon s} \int d \Omega_{\hat{S}} I_{N-1, M}^{R}(|\mathbf{j}-s \hat{s}|) .
$$

Letting $y=|\mathbf{j}-s \hat{s}|$, the angular integral in Eq. (2.29) can be reduced to an integral over $y$, giving finally

$$
I_{N, M}^{R}(j)=\lim _{\varepsilon \rightarrow 0}(8 j)^{-1} \int_{0}^{\infty} d s e^{-\varepsilon s} \int_{|j-s|}^{j+s} y d y I_{N-1, M}^{R}(y) .
$$

To simplify the fermionic recursion relation of Eq. (2.26b), we use the fact that the components of $\mathbf{r}(\psi)$ obey the identity

$$
r_{i} r_{j}=-8 \delta_{i j} \psi_{0} \psi_{1} \psi_{2} \psi_{3}:
$$

Developing $I_{N, M-1}^{R}(|\mathbf{j}-\mathbf{r}|)$ in a power series in $\mathbf{r}$, only the term of order $r^{2}$ survives in the Grassmann integral, giving

$$
I_{N, M}^{R}(j)=\int d \psi \frac{1}{2}\left(\mathbf{r}(\psi) \cdot \nabla_{\mathbf{j}}\right)^{2} I_{N, M-1}^{R}(j)=-4 \frac{1}{j^{2}} \frac{d}{d j} j^{2} \frac{d}{d j} I_{N, M-1}^{R}(j) .
$$

A simple induction in $N$ and $M$ now shows that the recursion relations of Eq. (2.30) and Eq. (2.32), and the initial condition [cf. Eq. (2.24b)]

$$
I_{0,0}^{R}(j)=\cos j,
$$

have the unique solution

$$
I_{N, M}^{R}(j)=4^{M-N}\left[\cos j-\frac{2(N-M)}{j} \sin j\right] .
$$

To conclude this section, we use Eq. (2.16) to derive a formula for the quaternionic delta function

$$
\delta(\phi, \psi)=\prod_{i=1}^{M} \delta\left(\phi_{0}^{i}\right) \delta\left(\phi_{1}^{i}\right) \delta\left(\phi_{2}^{i}\right) \delta\left(\phi_{3}^{i}\right) \psi_{0}^{i} \psi_{1}^{i} \psi_{2}^{i} \psi_{3}^{i},
$$

which is defined by the property (for arbitrary smooth $F$ )

$$
\left(\prod_{i=1}^{M} \int d \phi^{i} d \psi^{i}\right) \delta(\phi, \psi) F(\{\phi, \psi\})=F(\{0,0\}) .
$$

Let us consider Eq. (2.16) with $A=a e_{3}, B=b, \Omega=0$, and with $\phi$ interchanged with $u, \psi$ interchanged $\xi$, for which it reads

$$
\begin{aligned}
& \lim _{\varepsilon \rightarrow 0}\left(\prod_{i=1}^{M} \int d u^{i} d \xi^{i}\right)\left(4 \pi^{2}\right)^{-M} \exp \left(-a \bar{u} e_{3} u-b \bar{\xi} \xi-\varepsilon \bar{u} u+\bar{\phi} u-\bar{u} \phi+\bar{\psi} \xi+\bar{\xi} \psi\right) \\
& \quad=\left(b^{2} / a^{2}\right)^{M} \exp \left(a^{-1} \bar{\phi} e_{3} \phi+b^{-1} \bar{\psi} \psi\right) .
\end{aligned}
$$

We will now show that in the limit $a, b \rightarrow 0$, the right-hand side of Eq. (2.37) is proportional to the quaternionic delta function. Consider the integral

$$
\left(\prod_{i=1}^{M} \int d \phi^{i} d \psi^{i}\right)\left(4 \pi^{2}\right)^{-M}\left(b^{2} / a^{2}\right)^{M} \exp \left(a^{-1} \bar{\phi} e_{3} \phi+b^{-1} \bar{\psi} \psi\right) F(\{\phi, \psi\})
$$


Rescaling $\phi \rightarrow a^{1 / 2} \phi, \psi \rightarrow b^{1 / 2} \psi$, this becomes

$$
\left(\prod_{i=1}^{M} \int d \phi^{i} d \psi^{i}\right)\left(4 \pi^{2}\right)^{-M} \exp \left(\bar{\phi} e_{3} \phi+\bar{\psi} \psi\right) F\left(\left\{a^{1 / 2} \phi, b^{1 / 2} \psi\right\}\right),
$$

and expanding $F$ in a Taylor series and applying Eq. (2.16) to the leading term gives

$$
F(\{0,0\})+O\left(a^{1 / 2}, b^{1 / 2}\right) .
$$

Hence we conclude that

$$
\begin{aligned}
\delta(\phi, \psi)= & \lim _{a, b, \varepsilon \rightarrow 0}\left(\prod_{i=1}^{M} \int d u^{i} d \xi^{i}\right)(2 \pi)^{-4 M} \\
& \cdot \exp \left(-a \bar{u} e_{3} u-b \bar{\xi} \xi-\varepsilon \bar{u} u+\bar{\phi} u-\bar{u} \phi+\bar{\psi} \xi+\bar{\xi} \psi\right) .
\end{aligned}
$$

We will henceforth write this formula with the infinitesimals $a, b, \varepsilon$ suppressed. Since the integration measure $d u^{i} d \xi^{i}$ is invariant under the rescaling $u \rightarrow u c, \xi \rightarrow \xi c$ for any $c,{ }^{5}$ it is convenient to rescale by $c=\frac{1}{2}$ and to write Eq. (2.41) as

$$
\begin{aligned}
\delta(\phi, \psi) & =\left(\prod_{i=1}^{M} \int d u^{i} d \xi^{i}\right)(2 \pi)^{-4 M} e^{\Phi}=\left(\prod_{i=1}^{M} \int d u^{i} d \xi^{i}\right)(2 \pi)^{-4 M} \cosh \Phi, \\
\Phi & =\frac{1}{2} \sum_{i=1}^{M}\left(\overline{\phi^{i}} u^{i}-\bar{u}^{i} \phi^{i}+\bar{\psi}^{i} \xi^{i}+\overline{\xi^{i}} \psi^{i}\right) .
\end{aligned}
$$

As an explicit check on the above reasoning, let us specialize to the case $M=1$ and verify that

$$
\psi_{0} \psi_{1} \psi_{2} \psi_{3}=\int d \phi \delta(\phi, \psi)=\lim _{a, b \rightarrow 0} \int d \phi\left(b^{2} / a^{2}\right) \exp \left(a^{-1} \bar{\phi} e_{3} \phi+b^{-1} \bar{\psi} \psi\right)
$$

According to Eqs. (2.11)-(2.14), the right-hand side of Eq. (2.43) is given by

$$
\lim _{b \rightarrow 0} \frac{b^{2}}{4} L_{1}\left(b^{-1} \bar{\psi} \psi\right)=\lim _{b \rightarrow 0} \frac{b^{2}}{4}\left[-1+\frac{1}{3}\left(b^{-1} \bar{\psi} \psi\right)+\frac{1}{6}\left(b^{-1} \bar{\psi} \psi\right)^{2}\right],
$$

with the series terminating because $(\bar{\psi} \psi)^{3}=0$. In the limit of vanishing $b$ only the quadratic term contributes, giving

$$
\frac{1}{24}(\bar{\psi} \psi)^{2}=\frac{1}{24}(\mathbf{r} \cdot \mathbf{e})^{2}=-\frac{1}{24} \mathbf{r}^{2}=\psi_{0} \psi_{1} \psi_{2} \psi_{3},
$$

where we have used Eqs. (2.25) and (2.31).

It is also instructive to write down the complex analog of Eq. (2.42). In the complex case all imaginary quantities commute, and so we can treat the bosonic

\footnotetext{
${ }^{5}$ The scale invariance of the measure can be used to show that the reexponentiation property exhibited in Eq. (2.16) has the following generalization to a wide class of functions. Let $f(\kappa)$ be a generalized "positive frequency" function of the imaginary quaternion $\kappa$, defined by $f(\kappa)$ $=\int_{0}^{\infty} d \mu \varrho(\mu) e^{\mu \kappa}$, with $\mu$ real and with $\varrho(\mu)$ a quaternion-valued measure. Then $\int d \phi d \psi\left(4 \pi^{2}\right)^{-1} f\left(\kappa+\bar{\phi} e_{3} \phi+\bar{\psi} \psi\right)=f(\kappa)$
} 
and fermionic cases separately, and we lose no generality by taking $M=1$. In the bosonic case we have

$$
\begin{gathered}
\delta\left(\phi_{0}\right) \delta\left(\phi_{1}\right)=\frac{1}{(2 \pi)^{2}} \int d u_{0} d u_{1} e^{i\left(\phi_{0} u_{1}-\phi_{1} u_{0}\right)}=\frac{1}{(2 \pi)^{2}} \int d u_{0} d u_{1} e^{\Phi}, \\
\Phi=\frac{1}{2}(\bar{\phi} u-\bar{u} \phi), \quad \phi=\phi_{0}+i \phi_{1}, \quad u=u_{0}+i u_{1},
\end{gathered}
$$

and similarly in the fermionic case

$$
\begin{gathered}
\psi_{0} \psi_{1}=\int d \xi_{1} d \xi_{0} e^{i\left(\psi_{0} \xi_{1}-\psi_{1} \xi_{0}\right)}=\int d \xi_{1} d \xi_{0} e^{\Phi}, \\
\Phi=\frac{1}{2}(\bar{\psi} \xi+\xi \psi), \quad \psi=\psi_{0}+i \psi_{1}, \quad \xi=\xi_{0}+i \xi_{1} .
\end{gathered}
$$

These equations show that Eq. (2.42) should be thought of as a quaternionic analog of the Fourier representation for the delta function in the complex case. This analog has the surprising feature of requiring the inclusion of quaternionic Grassmann variables!

\section{Sum Over Histories Approach to Quaternionic Field Theory}

Let us now turn to the central problem of formulating the dynamics of a quaternionic field theory. We consider a system containing $M$ bosonic and $M$ Grassmann quaternion degrees of freedom, and take as our fundamental postulate the assumption that the quantum mechanical transformation function for an infinitesimal time interval $\Delta t=t_{j+1}-t_{j}$ has the form

$$
\begin{aligned}
& \left\langle\left\{\phi_{j+1}, \psi_{j+1}\right\}, t_{j+1} \mid\left\{\phi_{j}, \psi_{j}\right\}, t_{j}\right\rangle \\
& \quad=C^{-1} \exp \left[\Delta t \tilde{L}\left(\left\{\phi_{j+1 / 2}, \phi_{j+1 / 2}, \psi_{j+1 / 2}, \dot{\psi}_{j+1 / 2}\right\}, t_{j}+\Delta t / 2\right)\right],
\end{aligned}
$$

with $\tilde{L}$ a quaternion-imaginary Lagrangian, $\tilde{L}=\tilde{L}_{1} e_{1}+\tilde{L}_{2} e_{2}+\tilde{L}_{3} e_{3}$. The arguments of $\tilde{L}$ in Eq. (3.1) are to be evaluated in accordance with the trapezoidal rule at the midpoint of the interval [see Eq. (4.3) below]. Equation (3.1) is of course simply the natural quaternionic generalization of Dirac's famous observation [8] in the complex case, which forms the basis for Feynman's [9] sum-over-histories formulation of complex quantum mechanics. The quaternion imaginary quantity $\tilde{L}$ is the analog of the complex quantum mechanical $i L$; as pointed out in Sect. 1 , we cannot convert $\tilde{L}$ to a quaternion-real quantity by multiplying by an imaginary unit.

Compounding $N$ infinitesimal transformations, we get for finite time evolution,

$$
\begin{gathered}
\left\langle\left\{\phi_{N}, \psi_{N}\right\}, t_{N} \mid\left\{\phi_{0}, \psi_{0}\right\}, t_{0}\right\rangle=\left[\prod_{j=1}^{N-1}\left(\prod_{i=1}^{M} \int d \phi_{j}^{i} d \psi_{j}^{i}\right)\right] \\
\cdot C^{-1} \exp [\Delta t \tilde{L}(N-1 / 2)] C^{-1} \exp [\Delta t \tilde{L}(N-3 / 2)] \ldots C^{-1} \exp [\Delta t \tilde{L}(1 / 2)]
\end{gathered}
$$

in an abbreviated notation according to which the exponent in Eq. (3.1) would be written as $\Delta t \tilde{L}(j+1 / 2)$. In passing to the continuum limit, we must take into account the fact that the infinitesimal phases in Eq. (3.2) do not commute, and hence the product in Eq. (3.2) is not the exponential of the Riemann sum of exponents, as it is in the complex case. Formally, we can accomplish this by giving 
the quaternion units $e_{a}$ a time label, $e_{a} \rightarrow e_{a}(t)$, and defining a time-ordering operation $T$ as one which orders the $e_{a}$ 's with the later time on the left, giving the functional integral formula

$$
\left\langle\left\{\phi_{N}, \psi_{N}\right\}, t_{N} \mid\left\{\phi_{0}, \psi_{0}\right\}, t_{0}\right\rangle=\mathrm{const} \times \int d[\phi] d[\psi] T \exp \left[\int_{t_{0}}^{t_{N}} d t e_{a}(t) \tilde{L}_{a}(t)\right] .
$$

This construction guarantees that the transformation functions satisfy the quantum mechanical composition law (the principle of superposition of wave amplitudes)

$$
\begin{aligned}
\left\langle\left\{\phi_{N}, \psi_{N}\right\}, t_{N} \mid\left\{\phi_{0}, \psi_{0}\right\}, t_{0}\right\rangle= & \left(\prod_{i=1}^{M} \int d \phi_{I}^{i} d \psi_{I}^{i}\right)\left\langle\left\{\phi_{N}, \psi_{N}\right\}, t_{N} \mid\left\{\phi_{I}, \psi_{I}\right\}, t_{I}\right\rangle \\
& \cdot\left\langle\left\{\phi_{I}, \psi_{I}\right\}, t_{I} \mid\left\{\phi_{0}, \psi_{0}\right\}, t_{0}\right\rangle
\end{aligned}
$$

for any arbitrary intermediate time $t_{I}$.

At this point we will assume a specific functional form for $\tilde{L}$,

$$
\begin{gathered}
\tilde{L}(\{\phi, \dot{\phi}, \psi, \dot{\psi}\}, t)=\tilde{L}_{\mathrm{kin}}-\tilde{V}(\{\phi, \psi\}, t), \\
\tilde{L}_{\mathrm{kin}}=\frac{1}{2} \sum_{i=1}^{M}\left[\bar{\phi}^{i} e_{3} \phi^{i}+\bar{\psi}^{i} \dot{\psi}^{i}-\bar{A}^{i}(\{\phi, \psi\}, t) e_{3} \phi^{i}-\bar{\phi}^{i} e_{3} A^{i}(\{\phi, \psi\}, t)\right. \\
\left.-\bar{\beta}^{i}(\{\phi, \psi\}, t) \dot{\psi}^{i}-\bar{\psi}^{i} \beta^{i}(\{\phi, \psi\}, t)\right],
\end{gathered}
$$

with $\tilde{V}$ quaternion imaginary, and with $A^{i}$ and $\beta^{i}$, respectively, general bosonic and Grassmann quaternion velocity ("vector") potentials. The term in $\widetilde{L}_{\mathrm{kin}}$ quadratic in time derivatives clearly has a form motivated by the Gaussian integral formula of Eq. (2.16), with $A=e_{3}$ and $B=1$. The use of the quaternion unit $e_{3}$ is arbitrary; by the gauge transformation $\phi^{i} \rightarrow q \phi^{i}$ with $q$ a constant quaternion with $\bar{q} q=1$, which is an invariance [6] of the integration measure, $e_{3}$ can be converted to the general quaternionic imaginary unit $\bar{q} e_{3} q$, while preserving the general structure of Eq. (3.5). Hence Eq. (3.5) is consistent with the principle of quaternion covariance enunciated by Finkelstein et al. [10], which states that quaternionic quantum mechanics should not pick out a preferred quaternion frame. (This would not be the case had we instead used $e_{3} \bar{\phi} \dot{\phi}$ to get a quaternion-imaginary quantity, since the imaginary unit $e_{3}$ would then be unaffected by gauge transformations of $\phi$.) The fermion kinetic term in Eq. (3.5) is unconventional in that its leading term is second order in time derivatives. The Grassmann Hilbert space structure implied by this, and the question of whether our construction can be extended to first-order fermion actions (probably not), are discussed in detail in Sect. 9 below.

Before proceeding to applications of Eqs. (3.1)-(3.5), one final issue deserves comment. The alert reader will have noticed that the conjugation convention which we have adopted for fermion fields is opposite to that conventionally used in complex field theory, where one takes $\bar{\psi} \psi=+\bar{\psi} \psi$, and where the corresponding term in $i L$ would be $i \bar{\psi} \psi \propto r e a l \times \psi_{0} \psi_{1}$, as contrasted with $\bar{\psi} \psi \propto r e a l \times i \psi_{0} \psi_{1}$ in our convention. This point is discussed in detail in Sect. 9, where we show that because total fermion number is governed by a superselection rule, complex field theories have the same physical content in either convention. The convention which we have adopted is necessary in the quaternionic case, because since the imaginary units do not commute, it is essential that there be no imaginaries hidden in the definition of the Grassmann variables. 


\section{Derivation of the Schrödinger Equation from the Functional Integral}

Let us now use the functional integration formalism of the preceding section to derive the Schrödinger equation satisfied by the wave function

$$
\Psi(\{\phi, \psi\}, t) \equiv\langle\{\phi, \psi\}, t \mid \Psi\rangle .
$$

According to Eqs. (3.1)-(3.4) we have

$$
\begin{aligned}
& \Psi(\{\phi, \psi\}, t+\Delta t)=\left(\prod_{i=1}^{M} \int d \phi_{0}^{i} d \psi_{0}^{i}\right)\left\langle\{\phi, \psi\}, t+\Delta t \mid\left\{\phi_{0}, \psi_{0}\right\}, t\right\rangle\left\langle\left\{\phi_{0}, \psi_{0}\right\}, t \mid \Psi\right\rangle \\
= & \left(\prod_{i=1}^{M} \int d \phi_{0}^{i} d \psi_{0}^{i}\right) C^{-1} \exp \left[\Delta t \tilde{L}\left(\left\{\phi_{1 / 2}, \phi_{1 / 2}, \psi_{1 / 2}, \dot{\psi}_{1 / 2}\right\}, t+\Delta t / 2\right)\right] \Psi\left(\left\{\phi_{0}, \psi_{0}\right\}, t\right),
\end{aligned}
$$

with

$$
\begin{array}{cl}
\phi_{1 / 2}^{i}=\frac{1}{2}\left(\phi^{i}+\phi_{0}^{i}\right), & \psi_{1 / 2}^{i}=\frac{1}{2}\left(\psi^{i}+\psi_{0}^{i}\right), \\
\phi_{1 / 2}^{i}=\left(\phi^{i}-\phi_{0}^{i}\right) / \Delta t, & \dot{\psi}_{1 / 2}^{i}=\left(\psi^{i}-\psi_{0}^{i}\right) / \Delta t .
\end{array}
$$

Making the change of integration variables $\phi_{0}^{i}=\phi^{i}+(2 \Delta t)^{1 / 2} \eta^{i}$, $\psi_{0}^{i}=\psi^{i}+(2 \Delta t)^{1 / 2} \zeta^{i}$, and substituting Eq. (3.5) for $\tilde{L}$, the right-hand side of Eq. (4.2) becomes

$$
\begin{gathered}
\left(\prod_{i=1}^{M} \int d \eta^{i} d \zeta^{i}\right) C^{-1} \exp \left[\Delta t \tilde{L}^{\sharp}\right] \Psi^{\sharp}, \\
\Delta t \tilde{L}^{\sharp}=\sum_{i=1}^{M}\left\{\bar{\eta}^{i} e_{3} \eta^{i}+\bar{\zeta} \zeta^{i}+\frac{1}{2}\left[(2 \Delta t)^{1 / 2}+\Delta t \mathfrak{D}\right]\right. \\
\cdot\left[\bar{A}^{i}(\{\phi, \psi\}, t) e_{3} \eta^{i}+\bar{\eta}^{i} e_{3} A^{i}(\{\phi, \psi\}, t)\right. \\
\left.\left.+\bar{\beta}^{i}(\{\phi, \psi\}, t) \zeta^{i}+\bar{\zeta}^{i} \beta^{i}(\{\phi, \psi\}, t)\right]\right\}-\Delta t \tilde{V}(\{\phi, \psi\}, t)+O\left((\Delta t)^{3 / 2}\right), \\
\Psi^{\sharp}=\left[1+(2 \Delta t)^{1 / 2} \mathfrak{D}+\Delta t \mathfrak{D}^{2}\right] \Psi(\{\phi, \psi\}, t)+O\left((\Delta t)^{3 / 2}\right), \\
\mathfrak{D}=\sum_{i=1}^{M} \sum_{a=0}^{3}\left(\eta_{a}^{i} \frac{\partial}{\partial \phi_{a}^{i}}+\zeta_{a}^{i} \frac{\partial}{\partial \psi_{a}^{i}}\right) .
\end{gathered}
$$

To simplify our notation in what follows, we will henceforth suppress the arguments $\{\phi, \psi\}, t$ of the potentials and of $\Psi$, and use a summation convention for repeated indices $i, j$ and $a, b$. Let us now make the change of integration variables,

$$
\begin{aligned}
\eta^{i} \rightarrow \eta^{i}=\eta^{i}+\frac{1}{2}\left[(2 \Delta t)^{1 / 2}+\Delta t \mathfrak{D}\right] A^{i}, & \bar{\eta}^{i} \rightarrow \bar{\eta}^{i}=\bar{\eta}^{i}+\frac{1}{2}\left[(2 \Delta t)^{1 / 2}+\Delta t \mathfrak{D}\right] \bar{A}^{i}, \\
\zeta^{i} \rightarrow \zeta^{i}=\zeta^{i}+\frac{1}{2}\left[(2 \Delta t)^{1 / 2}+\Delta t \mathfrak{D}\right] \beta^{i}, & \overline{\zeta^{i}} \rightarrow \bar{\zeta}^{\prime i}=\bar{\zeta}^{i}+\frac{1}{2}\left[(2 \Delta t)^{1 / 2}+\Delta t \mathfrak{D}\right] \bar{\beta}^{i} .
\end{aligned}
$$

In terms of these new variables, $\Delta t \tilde{L}^{\sharp}$ and $\Psi^{\sharp}$ become

$$
\begin{gathered}
\Delta t \tilde{L}^{\sharp}=\bar{\eta}^{i} e_{3} \eta^{\prime i}+\bar{\zeta}^{i} \zeta^{i}-\Delta t\left[\tilde{V}+\frac{1}{2}\left(\bar{A}^{i} e_{3} A^{i}+\bar{\beta}^{i} \beta^{i}\right)\right]+O\left((\Delta t)^{3 / 2}\right), \\
\Psi^{\sharp}=\left[1+(2 \Delta t)^{1 / 2} \mathfrak{D}^{\prime}-\Delta t\left(A_{a}^{i} \frac{\partial}{\partial \phi_{a}^{i}}+\beta_{a}^{i} \frac{\partial}{\partial \psi_{a}^{i}}\right)+\Delta t(\mathfrak{D})^{2}\right] \Psi+O\left((\Delta t)^{3 / 2}\right), \\
\mathfrak{D}^{\prime}=\eta_{a}^{i} \frac{\partial}{\partial \phi_{a}^{i}}+\zeta_{a}^{i} \frac{\partial}{\partial \psi_{a}^{i}},
\end{gathered}
$$


while the integration measure becomes

$$
\prod_{i=1}^{M} \int d \eta^{i} d \zeta^{i}=\prod_{i=1}^{M} \int d \eta^{\prime i} d \zeta^{\prime i} J
$$

with $J$ the Jacobian of the transformation of Eq. (4.5). To order $\Delta t$ accuracy, the inverse transformation is

$$
\begin{aligned}
& \left.\eta_{a}^{i}=\eta_{a}^{i}-\frac{1}{2}\left[(2 \Delta t)^{1 / 2}+\Delta t \mathfrak{D}^{\prime}\right] A_{a}^{i}+O(\Delta t)^{3 / 2}\right), \\
& \zeta_{a}^{i}=\zeta_{a}^{i}-\frac{1}{2}\left[(2 \Delta t)^{1 / 2}+\Delta t \mathfrak{D}^{\prime}\right] \beta_{a}^{i}+O\left((\Delta t)^{3 / 2}\right),
\end{aligned}
$$

which implies that

$$
\begin{aligned}
& \frac{\partial}{\partial \eta_{b}^{\prime j}} \eta_{a}^{i}=\delta^{i j} \delta_{a b}-\frac{1}{2} \Delta t \frac{\partial}{\partial \phi_{b}^{j}} A_{a}^{i}+O\left((\Delta t)^{3 / 2}\right), \\
& \frac{\partial}{\partial \zeta_{b}^{\prime j}} \zeta_{a}^{i}=\delta^{i j} \delta_{a b}-\frac{1}{2} \Delta t \frac{\partial}{\partial \psi_{b}^{j}} \beta_{a}^{i}+O\left((\Delta t)^{3 / 2}\right) .
\end{aligned}
$$

Hence to requisite order the Jacobian is

$$
J=\operatorname{det}\left(\frac{\partial}{\partial \eta_{b}^{\prime j}} \eta_{a}^{i}\right) \operatorname{det}^{-1}\left(\frac{\partial}{\partial \zeta_{b}^{\prime j}} \zeta_{a}^{i}\right)=1-\frac{1}{2} \Delta t \frac{\partial}{\partial \phi_{a}^{i}} A_{a}^{i}+\frac{1}{2} \Delta t \frac{\partial}{\partial \psi_{a}^{i}} \beta_{a}^{i}+O\left((\Delta t)^{3 / 2}\right) .
$$

At this stage there is no confusion if we drop the primes on the new integration variables. Taking into account the fact that when Eq. (4.6) for $\Psi^{\sharp}$ is substituted back into Eq. (4.4), the term $\mathfrak{D}^{\prime} \Psi$ and the fermion-boson cross-term part of $\left(\mathfrak{D}^{\prime}\right)^{2} \Psi$ both integrate to zero (since the exponent $\Delta t \tilde{L}^{\#}$ is even in the shifted integration variables), we have altogether

$$
\begin{aligned}
& \Psi(\{\phi, \psi\}, t+\Delta t)=\Psi+\Delta t \frac{\partial}{\partial t} \Psi+O\left((\Delta t)^{2}\right)=\left(\prod_{i=1}^{M} \int d \eta^{i} d \zeta^{i}\right) C^{-1} \\
& \cdot \exp \left\{\bar{\eta}^{i} e_{3} \eta^{i}+\overline{\zeta^{i}} \zeta^{i}-\Delta t\left[\tilde{V}+\frac{1}{2}\left(\bar{A}^{i} e_{3} A^{i}+\overline{\beta^{i}} \beta^{i}\right)\right]+O\left((\Delta t)^{3 / 2}\right)\right\} \\
& .\left\{\left[1-\frac{1}{2} \Delta t\left(\frac{\partial}{\partial \phi_{a}^{i}} A_{a}^{i}+A_{a}^{i} \frac{\partial}{\partial \phi_{a}^{i}}\right)+\frac{1}{2} \Delta t\left(\frac{\partial}{\partial \psi_{a}^{i}} \beta_{a}^{i}-\beta_{a}^{i} \frac{\partial}{\partial \psi_{a}^{i}}\right)\right] \Psi\right. \\
& \left.\quad+\Delta t \eta_{a}^{i} \eta_{b}^{j} \frac{\partial}{\partial \phi_{a}^{i}} \frac{\partial}{\partial \phi_{b}^{j}} \Psi-\Delta t \zeta_{a}^{i} \zeta_{b}^{j} \frac{\partial}{\partial \psi_{a}^{i}} \frac{\partial}{\partial \psi_{b}^{j}} \Psi+O\left((\Delta t)^{3 / 2}\right)\right\} .
\end{aligned}
$$

Note that if we had omitted the velocity potentials we would already have reached this stage at Eq. (4.4).

We can now proceed by applying the Gaussian integration formula of Eq. (2.16), with $A=-e_{3}$ and $B=-1$. To reproduce the $\Psi$ term on the left-hand side of Eq. (4.11) we must clearly take $C=\left(4 \pi^{2}\right)^{M}$, and then the first line of the curly bracket (which is independent of the integration variables $\eta, \zeta$ ) contributes

$$
\begin{aligned}
& \exp \left\{-\Delta t\left[\tilde{V}+\frac{1}{2}\left(\bar{A}^{i} e_{3} A^{i}+\bar{\beta}^{i} \beta^{i}\right)\right]\right\} \\
& \quad \cdot\left[1-\frac{1}{2} \Delta t\left(\frac{\partial}{\partial \phi_{a}^{i}} A_{a}^{i}+A_{a}^{i} \frac{\partial}{\partial \phi_{a}^{i}}\right)+\frac{1}{2} \Delta t\left(\frac{\partial}{\partial \psi_{a}^{i}} \beta_{a}^{i}-\beta_{a}^{i} \frac{\partial}{\partial \psi_{a}^{i}}\right)\right] \Psi \\
& \quad+O\left((\Delta t)^{3 / 2}\right)=\Psi-\Delta t \tilde{H}_{\mathrm{pot}} \Psi,
\end{aligned}
$$


with

$$
\begin{aligned}
\widetilde{H}_{\mathrm{pot}}= & \tilde{V}(\{\phi, \psi\}, t)+\frac{1}{2} \sum_{i=1}^{M}\left[\bar{A}^{i}(\{\phi, \psi\}, t) e_{3} A^{i}(\{\phi, \psi\}, t)\right. \\
& \left.+\bar{\beta}^{i}(\{\phi, \psi\}, t) \beta^{i}(\{\phi, \psi\}, t)\right] \\
& +\frac{1}{2} \sum_{i=1}^{M} \sum_{a=0}^{3}\left[\frac{\partial}{\partial \phi_{a}^{i}} A_{a}^{i}(\{\phi, \psi\}, t)+A_{a}^{i}(\{\phi, \psi\}, t)\right. \\
& \left.\cdot \frac{\partial}{\partial \phi_{a}^{i}}-\frac{\partial}{\partial \psi_{a}^{i}} \beta_{a}^{i}(\{\phi, \psi\}, t)+\beta_{a}^{i}(\{\phi, \psi\}, t) \frac{\partial}{\partial \psi_{a}^{i}}\right] .
\end{aligned}
$$

The second line of the curly bracket in Eq. (4.11) can be evaluated by noting that terms with $i \neq j$ integrate to zero; for each $i$ we can then use Eq. (2.16) with $M \rightarrow M-1$ to integrate out $M-1$ degrees of freedom, yielding

$$
-\Delta t \tilde{H}_{\text {kin }} \Psi, \quad \tilde{H}_{\text {kin }}=-I_{a b}^{B} \frac{\partial}{\partial \phi_{a}^{i}} \frac{\partial}{\partial \phi_{b}^{i}}+I_{a b}^{F} \frac{\partial}{\partial \psi_{a}^{i}} \frac{\partial}{\partial \psi_{b}^{i}},
$$

with

$I_{a b}^{B}=\int d \eta d \zeta\left(4 \pi^{2}\right)^{-1} \eta_{a} \eta_{b} \exp \left(\bar{\eta} e_{3} \eta+\overline{\zeta \zeta}\right), \quad I_{a b}^{F}=\int d \eta d \zeta\left(4 \pi^{2}\right)^{-1} \zeta_{a} \zeta_{b} \exp \left(\bar{\eta} e_{3} \eta+\overline{\zeta \zeta}\right)$.

To compute the integrals in Eq. (4.15), we first use Eq. (2.11) to evaluate the fermionic integral in $I_{a b}^{B}$ and the bosonic integral in $I_{a b}^{F}$. Restoring the infinitesimal convergence factor to $I_{a b}^{B}$, we get

$$
I_{a b}^{B}=\pi^{-2} \lim _{\varepsilon \rightarrow 0} \int d \eta \exp (-\varepsilon \bar{\eta} \eta) \eta_{a} \eta_{b} L_{-1}\left(\bar{\eta} e_{3} \eta\right), \quad I_{a b}^{F}=4^{-1} \int d \zeta \zeta_{a} \zeta_{b} L_{1}(\zeta \zeta)
$$

The integral for $I_{a b}^{F}$ receives a contribution only from the term $\overline{\zeta \zeta} / 3$ in the power series expansion of $L_{1}$ [Eqs. (2.14) and (2.44)], giving [cf. Eq. (2.25)]

$$
I_{a b}^{F}=\frac{1}{12} \int d \zeta_{a} \zeta_{b}\left[2 e_{1}\left(\zeta_{0} \zeta_{1}-\zeta_{2} \zeta_{3}\right)+2 \text { cyclic permutations }\right],
$$

from which we find

$$
I_{01}^{F}=-I_{10}^{F}=-I_{23}^{F}=I_{32}^{F}=-\frac{1}{6} e_{1},
$$

with the remaining $I^{F \prime} s$ given by cyclic permutation of the $1,2,3$ indices. To compute $I_{a b}^{B}$, we substitute Eq. (2.12) for $L_{-1}$ and make the change of integration variables of Eq. (2.27), giving

$$
\begin{gathered}
I_{a b}^{B}=\frac{1}{4 \pi^{2}}\left(I_{1} J_{1 a b}+I_{2} J_{2 a b}\right), \quad I_{1}=\lim _{\varepsilon \rightarrow 0} \int_{0}^{\infty} R^{5} d R e^{-\varepsilon R^{2}}\left(\cos R^{2}+\frac{2}{R^{2}} \sin R^{2}\right) \\
I_{2}=\lim _{\varepsilon \rightarrow 0} \int_{0}^{\infty} R^{5} d R e^{-\varepsilon R^{2}}\left(-\frac{d}{d R^{2}}\right)\left(\cos R^{2}+\frac{2}{R^{2}} \sin R^{2}\right) \\
J_{1 a b}=\int_{0}^{\pi} \sin \Theta d \Theta \int_{0}^{2 \pi} d \theta_{1} \int_{0}^{2 \pi} d \theta_{2} \Omega_{a} \Omega_{b} \\
J_{2 a b}=\int_{0}^{\pi} \sin \Theta d \Theta \int_{0}^{2 \pi} d \theta_{1} \int_{0}^{2 \pi} d \theta_{2} \Omega_{a} \Omega_{b} \\
\cdot\left[e_{1} \sin \Theta \sin \left(\theta_{1}-\theta_{2}\right)+e_{2} \sin \Theta \cos \left(\theta_{1}-\theta_{2}\right)+e_{3} \cos \Theta\right]
\end{gathered}
$$


with $\Omega_{a}$ the angular part of $\eta_{a}$,

$$
\begin{array}{ll}
\Omega_{0}=\cos \theta_{1} \cos \Theta / 2, & \Omega_{3}=\sin \theta_{1} \cos \Theta / 2, \\
\Omega_{1}=\cos \theta_{2} \sin \Theta / 2, & \Omega_{2}=\sin \theta_{2} \sin \Theta / 2 .
\end{array}
$$

We proceed first to evaluate the radial integrals. Substituting $R^{2}=u$, we have

$$
\begin{aligned}
I_{1} & =\lim \frac{1}{\varepsilon \rightarrow 0} \int_{0}^{\infty} u^{2} d u e^{-\varepsilon u}\left(\cos u+\frac{2}{u} \sin u\right) \\
& =\lim \frac{1}{2} \int_{0}^{\infty} d u e^{-\varepsilon u} \frac{d}{d u}\left(u^{2} \sin u\right) \\
& =\lim \frac{1}{2} \varepsilon \int_{0}^{\infty} d u e^{-\varepsilon u} u^{2} \sin u=0, \\
I_{2} & =\lim \left(-\frac{1}{2}\right) \int_{0}^{\infty} u^{2} d u e^{-\varepsilon u} \frac{d}{d u}\left(\cos u+\frac{2}{u} \sin u\right) \\
& =\lim \int_{0}^{\infty} d u e^{-\varepsilon u}(u \cos u+2 \sin u) \\
& =\lim \int_{0}^{\infty} d u e^{-\varepsilon u}\left[\frac{d}{d u}(u \sin u)+\sin u\right] \\
& =\lim \int_{0}^{\infty} d u e^{-\varepsilon u} \sin u=\lim \frac{1}{2 i}\left(\frac{1}{\varepsilon-i}-\frac{1}{\varepsilon+i}\right)=1 .
\end{aligned}
$$

Hence the quaternion-real part of $I_{a b}^{B}$ vanishes, and to complete the calculation we must evaluate the quaternion-imaginary angular integral $J_{2 a b}=J_{2 b a}$ for the 10 independent choices of $a, b$. These integrations are straightforward, and yield

$$
\begin{gathered}
I_{00}^{B}=I_{33}^{B}=-I_{11}^{B}=-I_{22}^{B}=\frac{1}{6} e_{3}, \quad I_{03}^{B}=I_{12}^{B}=0, \\
I_{01}^{B}=I_{23}^{B}=\frac{1}{6} e_{2}, \quad I_{02}^{B}=-I_{13}^{B}=-\frac{1}{6} e_{1} .
\end{gathered}
$$

Substituting Eqs. (4.18) and (4.22) into Eq. (4.14), we get

$$
\begin{aligned}
\tilde{H}_{\mathrm{kin}}= & -\frac{1}{6} \sum_{i=1}^{M}\left[2 e_{1}\left(\frac{\partial}{\partial \phi_{1}^{i}} \frac{\partial}{\partial \phi_{3}^{i}}-\frac{\partial}{\partial \phi_{0}^{i}} \frac{\partial}{\partial \phi_{2}^{i}}\right)+2 e_{2}\left(\frac{\partial}{\partial \phi_{0}^{i}} \frac{\partial}{\partial \phi_{1}^{i}}+\frac{\partial}{\partial \phi_{2}^{i}} \frac{\partial}{\partial \phi_{3}^{i}}\right)\right. \\
& \left.+e_{3}\left(\frac{\partial}{\partial \phi_{0}^{i}} \frac{\partial}{\partial \phi_{0}^{i}}+\frac{\partial}{\partial \phi_{3}^{i}} \frac{\partial}{\partial \phi_{3}^{i}}-\frac{\partial}{\partial \phi_{1}^{i}} \frac{\partial}{\partial \phi_{1}^{i}}-\frac{\partial}{\partial \phi_{2}^{i}} \frac{\partial}{\partial \phi_{2}^{i}}\right)\right] \\
& +\frac{1}{6} \sum_{i=1}^{M}\left[2 e_{1}\left(\frac{\partial}{\partial \psi_{2}^{i}} \frac{\partial}{\partial \psi_{3}^{i}}-\frac{\partial}{\partial \psi_{0}^{i}} \frac{\partial}{\partial \psi_{1}^{i}}\right)+2 e_{2}\left(\frac{\partial}{\partial \psi_{3}^{i}} \frac{\partial}{\partial \psi_{1}^{i}}-\frac{\partial}{\partial \psi_{0}^{i}} \frac{\partial}{\partial \psi_{2}^{i}}\right)\right. \\
& \left.+2 e_{3}\left(\frac{\partial}{\partial \psi_{1}^{i}} \frac{\partial}{\partial \psi_{2}^{i}}-\frac{\partial}{\partial \psi_{0}^{i}} \frac{\partial}{\psi_{3}^{i}}\right)\right]
\end{aligned}
$$


which by comparison with Eq. (2.25) can be rewritten as

$$
\begin{gathered}
\tilde{H}_{\mathrm{kin}}=-\frac{1}{6} \sum_{i=1}^{M}\left(\bar{D}_{\phi^{i}} e_{3} D_{\phi^{i}}+\bar{D}_{\psi^{i}} D_{\psi^{i}}\right), \quad D_{\phi} \equiv \frac{\partial}{\partial \phi_{0}}+e_{1} \frac{\partial}{\partial \phi_{1}}+e_{2} \frac{\partial}{\partial \phi_{2}}+e_{3} \frac{\partial}{\partial \phi_{3}}, \\
D_{\psi} \equiv \frac{\partial}{\partial \psi_{0}}+e_{1} \frac{\partial}{\partial \psi_{1}}+e_{2} \frac{\partial}{\partial \psi_{2}}+e_{3} \frac{\partial}{\partial \psi_{3}} .
\end{gathered}
$$

Finally, combining Eqs. (4.11), (4.12), and (4.14), we get the Schrödinger equation for $\Psi$,

$$
\frac{\partial \Psi}{\partial t}=-\tilde{H} \Psi, \quad \tilde{H}=\tilde{H}_{\mathrm{kin}}+\tilde{H}_{\mathrm{pot}}
$$

with $\tilde{H}_{\text {kin }}$ and $\tilde{H}_{\text {pot }}$ given by Eq. (4.24) and Eq. (4.13) respectively.

The fact that the general form of the quaternionic Schrödinger equation is independent of the number of modes $M$ is a direct consequence of the fact that, when the numbers of fermionic and bosonic modes are equal, the sourcedependence in the Gaussian integral formula of Eq. (2.11) remains exponential. What happens if we formulate a quaternionic quantum field theory with unequal numbers $N$ and $M$ of bosonic and fermionic degrees of freedom? We can still define a functional integral, as in the preceding section, and we can still derive a Schrödinger equation, as above, but the coefficients in the Schrödinger equation will now explicitly depend on $N$ and $M$. The one case, when $N \neq M$, where the form of the Schrödinger equation does not depend on the numbers of degrees of freedom is when $|M-N| \rightarrow \infty$. In this limit Eq. (2.12) implies that

$$
\frac{L_{M-N}(\mathbf{j} \cdot \mathbf{e})}{-2(M-N)} \rightarrow f(\mathbf{j} \cdot \mathbf{e}) \equiv \frac{\sin j}{j}+\frac{\mathbf{j} \cdot \mathbf{e}}{j}\left(\frac{\sin j}{j^{2}}-\frac{\cos j}{j}\right),
$$

with the function on the right a fixed point (up to normalization) of the recursion relations of Eqs. (2.30) and (2.32). We then get a Schrödinger equation in which $f(\mathbf{j} \cdot \mathbf{e})$ of Eq. (4.26) replaces $\exp (\mathbf{j} \cdot \mathbf{e})$ in analogs of Eqs. (4.12) and (4.15). A little calculation shows that the effect of this is to multiply the first line in Eq. (4.13) by 1/3, while leaving the second line in Eq. (4.13), and Eq. (4.24), unchanged (apart from the appropriate changes in the limits of the boson and fermion sums $\sum_{i}$ ). We do not pursue this direction further in this paper, but in principle it gives an alternative method of formulating a quaternionic field theory, and deserves detailed study.

\section{Derivation of the Functional Integral from the Schrödinger Equation}

In the preceding two sections we have very closely followed Feynman's [9] approach to quantum mechanics, in which the Lagrangian functional integral is taken as fundamental and the Schrödinger equation is derived as a consequence. In the modern literature the customary treatment is to proceed in reverse, starting from the transformation theory based on the quantum Hamiltonian and showing that this implies the functional integral formula for the transition amplitude for a finite time interval. As a check that Sects. 3 and 4 define a consistent quantum 
mechanical scheme, let us show that this reverse procedure works in the quaternionic case, by deriving Eq. (3.2) from Eq. (4.25). Since the details are quite complicated, we will consider only the simplified problem in which the velocity potentials $A^{i}$ and $\beta^{i}$ are neglected, and so we take as our starting point Eq. (4.25) with

$$
\tilde{H}=\tilde{H}_{\mathrm{kin}}+\tilde{V}(\{\phi, \psi\}, t) .
$$

We begin by noting that the reasoning used to derive the $\tilde{V}$ term in Eqs. (4.12)-(4.13) shows that for any smooth function $f$ we have

$$
\begin{aligned}
\left(\prod_{i=1}^{M} \int d \eta^{i} d \zeta^{i}\right)\left(4 \pi^{2}\right)^{-M} \exp \left[\sum_{i}\left(\bar{\eta}^{i} e_{3} \eta^{i}+\bar{\zeta} \zeta^{i}\right)-\Delta t \tilde{V}\right] f\left(\left\{(2 \Delta t)^{1 / 2} \eta,(2 \Delta t)^{1 / 2} \zeta\right\}\right) \\
=\exp (-\Delta t \tilde{V}) f(\{0,0\})+\Delta t \times(\tilde{V} \text {-independent terms })+O\left((\Delta t)^{3 / 2}\right) \\
=\left(\prod_{i=1}^{M} \int d \eta^{i} d \zeta^{i}\right)\left(4 \pi^{2}\right)^{-M} \exp \left[\sum_{i}\left(\bar{\eta}^{i} e_{3} \eta^{i}+\overline{\zeta^{2}} \zeta^{i}\right)\right] \\
\quad \cdot \exp (-\Delta t \tilde{V}) f\left(\left\{(2 \Delta t)^{1 / 2} \eta,(2 \Delta t)^{1 / 2} \zeta\right\}\right),
\end{aligned}
$$

and so within a functional integral we have the equivalence (denoted by $\leftrightarrow$ )

$$
\exp \left(\Delta t \tilde{L}_{\text {kin }}-\Delta t \tilde{V}\right) \leftrightarrow \exp \left(\Delta t \tilde{L}_{\text {kin }}\right) \exp (-\Delta t \tilde{V})
$$

Hence it suffices to prove the following modified version of Eq. (3.2),

$$
\begin{aligned}
& \left\langle\left\{\phi_{N}, \psi_{N}\right\}, t_{N} \mid\left\{\phi_{0}, \psi_{0}\right\}, t_{0}\right\rangle=\left[\prod_{j=1}^{N-1}\left(\prod_{i=1}^{M} \int d \phi_{j}^{i} d \psi_{j}^{i}\right)\right] \\
& \cdot\left(4 \pi^{2}\right)^{-M} \exp \left[\Delta t \tilde{L}_{\mathrm{kin}}(N-1 / 2)\right] \exp [-\Delta t \tilde{V}(N-1 / 2)] \\
& \cdot\left(4 \pi^{2}\right)^{-M} \exp \left[\Delta t \tilde{L}_{\mathrm{kin}}(N-3 / 2)\right] \exp [-\Delta t \tilde{V}(N-3 / 2)] \ldots\left(4 \pi^{2}\right)^{-M} \\
& \quad \cdot \exp \left[\Delta t \tilde{L}_{\mathrm{kin}}(1 / 2)\right] \exp [-\Delta t \tilde{V}(1 / 2)] .
\end{aligned}
$$

According to the transformation theory based on the quantum Hamiltonian of Eq. (5.1), we have

$$
\left\langle\left\{\phi_{N}, \psi_{N}\right\}, t_{N} \mid\left\{\phi_{0}, \psi_{0}\right\}, t_{0}\right\rangle=\left\langle\left\{\phi_{N}, \psi_{N}\right\}, t_{0}\left|\exp \left[-\tilde{H}\left(t_{N}-t_{0}\right)\right]\right|\left\{\phi_{0}, \psi_{0}\right\}, t_{0}\right\rangle .
$$

Applying the Trotter product formula [11] to the finite-time evolution operator $\exp \left[-\tilde{H}\left(t_{N}-t_{0}\right)\right]$, we have

$$
\begin{aligned}
& \exp \left[-\tilde{H}\left(t_{N}-t_{0}\right)\right]=\left\{\exp \left[-\left(\tilde{H}_{\mathrm{kin}}+\tilde{V}\right)\left(t_{N}-t_{0}\right) / N\right]\right\}^{N} \\
& \quad=\left\{\exp \left[-\tilde{H}_{\mathrm{kin}}\left(t_{N}-t_{0}\right) / N\right] \exp \left[-\tilde{V}\left(t_{N}-t_{0}\right) / N\right]+O\left(\frac{1}{N^{2}}\right)\right\}^{N},
\end{aligned}
$$

and so taking $\left(t_{N}-t_{0}\right) / N=\Delta t$, and inserting $N-1$ complete sets of intermediate states between the $N$ factors on the right-hand side of Eq. (5.5), we get

$$
\begin{aligned}
\left\langle\left\{\phi_{N}, \psi_{N}\right\}, t_{N} \mid\left\{\phi_{0}, \psi_{0}\right\}, t_{0}\right\rangle=\left[\prod_{j=1}^{N-1}\left(\prod_{i=1}^{M} \int d \phi_{j}^{i} d \psi_{j}^{i}\right)\right] \\
\quad \cdot\left\langle\left\{\phi_{N}, \psi_{N}\right\}, t_{0}\left|\exp \left(-\tilde{H}_{\mathrm{kin}} \Delta t\right) \exp (-\tilde{V} \Delta t)\right|\left\{\phi_{N-1}, \psi_{N-1}\right\}, t_{0}\right\rangle \\
\cdot\left\langle\left\{\phi_{N-1}, \psi_{N-1}\right\}, t_{0}\left|\exp \left(-\tilde{H}_{\mathrm{kin}} \Delta t\right) \exp (-\tilde{V} \Delta t)\right|\left\{\phi_{N-2}, \psi_{N-2}\right\}, t_{0}\right\rangle \ldots \\
\quad \cdot\left\langle\left\{\phi_{1}, \psi_{1}\right\}, t_{0}\left|\exp \left(-\tilde{H}_{\mathrm{kin}} \Delta t\right) \exp (-\tilde{V} \Delta t)\right|\left\{\phi_{0}, \psi_{0}\right\}, t_{0}\right\rangle .
\end{aligned}
$$


Hence to prove Eq. (5.3) it suffices to prove that

$$
\begin{aligned}
& \left\langle\{\phi, \psi\}, t\left|\exp \left(-\Delta t \tilde{H}_{\text {kin }}\right) \exp (-\Delta t \tilde{V})\right|\left\{\phi^{\prime}, \psi^{\prime}\right\}, t\right\rangle \\
& \quad=\left(4 \pi^{2}\right)^{-M} \exp \left(\Delta t \tilde{L}_{\text {kin }}\right) \exp (-\Delta t \tilde{V}) .
\end{aligned}
$$

Without loss of generality [12] the coordinate eigenstates $\left\{\left\{\phi^{\prime}, \psi^{\prime}\right\}, t\right\rangle$ can be taken to be quaternion real,

$$
e_{a}\left|\left\{\phi^{\prime}, \psi^{\prime}\right\}, t\right\rangle=\left|\left\{\phi^{\prime}, \psi^{\prime}\right\}, t\right\rangle e_{a}
$$

they are then eigenstates of $\tilde{V}$ and $\exp (-\Delta t \tilde{V})$ can be moved outside the ket and factored away. We can now use translation invariance to set $\left\{\phi^{\prime}, \psi^{\prime}\right\}=\{0,0\}$, and so we have reduced the path integral derivation to the problem of proving

$$
\left\langle\{\phi, \psi\}, t\left|\exp \left(-\Delta t \tilde{H}_{\mathrm{kin}}\right)\right|\{0,0\}, t\right\rangle=\left(4 \pi^{2}\right)^{-M} \exp \left[\sum_{i=1}^{M}\left(\bar{\phi}^{i} e_{3} \phi^{i}+\bar{\psi}^{i} \psi^{i}\right) / 2 \Delta t\right] \text {. }
$$

Since the proof of Eq. (5.9) is quite complicated, it will be instructive to first derive, by a method which generalizes to the quaternionic case, the analog of Eq. (5.9) which appears in the conventional complex path integral for a single degree of freedom,

$$
\left\langle x, t\left|\exp \left(-i \Delta t p^{2} / 2 m\right)\right| 0, t\right\rangle=\left(\frac{m}{2 \pi i \Delta t}\right)^{1 / 2} \exp \left(-\frac{m x^{2}}{2 i \Delta t}\right) .
$$

Writing $\lambda=i \Delta t / 2 m$, and using the coordinate representation form $p^{2}=-(\partial / \partial x)^{2}$, the left-hand side of Eq. (5.10) is

$$
\left\langle x, t\left|\exp \left(\lambda \partial^{2} / \partial x^{2}\right)\right| 0, t\right\rangle=\exp \left(\lambda \partial^{2} / \partial x^{2}\right)\langle x, t \mid 0, t\rangle \exp \left(-\lambda \partial^{2} / \partial x^{2}\right) .
$$

[Derivatives acting to the right of all $x$ 's vanish, so $\exp \left(-\lambda \partial^{2} / \partial x^{2}\right)$ is equivalent to 1 in Eq. (5.11).] The matrix element $\langle x, t \mid 0, t\rangle$ is just the Dirac delta function $\delta(x)$, which has the Fourier representation

$$
\langle x, t \mid 0, t\rangle=\delta(x)=\frac{1}{2 \pi} \int_{-\infty}^{\infty} d p e^{i p x},
$$

and so Eq. (5.11) becomes

$$
\exp \left(\lambda \partial^{2} / \partial x^{2}\right) \frac{1}{2 \pi} \int_{-\infty}^{\infty} d p e^{i p x} \exp \left(-\lambda \partial^{2} / \partial x^{2}\right)
$$

Let us first give an exact evaluation of Eq. (5.13), and then an approximate computation [with an error of order $O\left((\Delta t)^{2}\right)$ ] which is equivalent to the exact treatment inside a functional integral. For the exact evaluation, we bring the differential operator inside the exponent in Eq. (5.13) to give

$$
\begin{aligned}
& \frac{1}{2 \pi} \int_{-\infty}^{\infty} d p \exp \left[i p \exp \left(\lambda \partial^{2} / \partial x^{2}\right) x \exp \left(-\lambda \partial^{2} / \partial x^{2}\right)\right] \\
& \quad=\frac{1}{2 \pi} \int_{-\infty}^{\infty} d p \exp [i p(x+2 \lambda \partial / \partial x)] \\
& \quad=\frac{1}{2 \pi} \int_{-\infty}^{\infty} d p \exp \left(i p x-\lambda p^{2}\right)
\end{aligned}
$$


where we have again used the fact that $\partial / \partial x$ vanishes when acting to the right of all $x$ 's, and have employed the identities

$$
e^{B} A e^{-B}=A+[B, A], \quad e^{A+B}=e^{A-\frac{1}{2}[A, B]} e^{B},
$$

the first valid when $[B,[A, B]]=0$ and the second valid when $[A,[A, B]]$ $=[B,[A, B]]=0$. Finally, the standard Gaussian integral

$$
\int_{-\infty}^{\infty} d y e^{-a y^{2}+b y}=\left(\frac{\pi}{a}\right)^{1 / 2} e^{b^{2} / 4 a}
$$

allows us to do the final integral in Eq. (5.14), yielding

$$
\frac{1}{(4 \pi \lambda)^{1 / 2}} \exp \left(\frac{-x^{2}}{4 \lambda}\right)
$$

which is the right-hand side of Eq. (5.10).

In the quaternionic case the presence of non-commuting quaternionic units invalidates the identities of Eq. (5.15), and so an exact derivation in analogy with Eqs. (5.10)-(5.17) is not possible. However, to complete the derivation of Eqs. (5.1)-(5.9), an evaluation of Eq. (5.9) is needed only through terms of order $\Delta t$. There are two ways of seeing why this should be so. The first is to note that terms of order $(\Delta t)^{2}$ are of the same order as the error $O\left(1 / N^{2}\right)$ in the Trotter product formula, and so they formally do not contribute to the functional integral in the $\Delta t \rightarrow 0(N \rightarrow \infty)$ limit. The second way is to note that we need only evaluate the matrix element of Eq. (5.9) up to an equivalence $(\leftrightarrow)$ inside the functional integral. Two different forms of Eq. (5.9) are equivalent inside a functional integral if their $0^{\text {th }}, 1^{\text {st }}$, and $2^{\text {nd }}$ moments with respect to the coordinates $\phi^{i}, \psi^{i}$ agree, since these are all that enter into the Schrödinger equation derivation of the preceding section. Since $\tilde{H}_{\text {kin }}^{2}$ is fourth order in coordinate derivatives, terms of order $(\Delta t)^{2} \tilde{H}_{\text {kin }}^{2}$ and higher do not contribute to coordinate moments of Eq. (5.9) of degree $\leqq 2$, and so can be dropped from the derivation.

Applying these remarks to the complex analog, we must only prove the identity of Eq. (5.10) up to an error of order $(\Delta t)^{2}$, or equivalently, since

$$
\int_{-\infty}^{\infty} d x\left(1, x, x^{2}\right)\left(\lambda \frac{\partial^{2}}{\partial x^{2}}\right)^{2} \delta(x)=0
$$

we must prove

$$
\left\langle x, t\left|\exp \left(-i \Delta t p^{2} / 2 m\right)\right| 0, t\right\rangle \leftrightarrow\left(\frac{m}{2 \pi i \Delta t}\right)^{1 / 2} \exp \left(\frac{-m x^{2}}{2 i \Delta t}\right),
$$

where $F(x) \leftrightarrow 0$ iff

$$
\int_{-\infty}^{\infty} d x\left(1, x, x^{2}\right) F(x)=0
$$

It is now convenient to use a Fourier cosine representation for $\delta(x)$,

$$
\delta(x)=\frac{1}{2 \pi} \int_{-\infty}^{\infty} d p \cosh \Phi, \quad \Phi \equiv i p x .
$$


Developing Eq. (5.20) in a power series expansion, substituting into Eq. (5.11) and keeping only terms through first order in $\lambda \propto \Delta t$, we have

$$
\left\langle x, t\left|\exp \left(-i \Delta t p^{2} / 2 m\right)\right| 0, t\right\rangle \leftrightarrow \frac{1}{2 \pi} \int_{-\infty}^{\infty} d p \sum_{n=0}^{\infty} \frac{1}{(2 n) !}\left\{\left(\Phi^{2}\right)^{n}+\lambda\left[\partial^{2} / \partial x^{2},\left(\Phi^{2}\right)^{n}\right]\right\} .
$$

The commutator in Eq. (5.21) can be evaluated in terms of

$$
\lambda\left[\partial^{2} / \partial x^{2}, \Phi^{2}\right]=-2 p^{2} \lambda+\delta
$$

with $\delta$ the differential operator

$$
\delta=-4 p^{2} \lambda x \partial / \partial x
$$

which has the commutator with $\Phi^{2}$

$$
\left[\delta, \Phi^{2}\right]=-8 p^{2} \lambda \Phi^{2} .
$$

So the power series on the right-hand side of Eq. (5.21) becomes

$$
\begin{aligned}
\sum_{n=0}^{\infty} \frac{1}{(2 n) !}\left[\left(\Phi^{2}\right)^{n}+n\left(\Phi^{2}\right)^{n-1}\left(-2 p^{2} \lambda\right)+\delta\left(\Phi^{2}\right)^{n-1}\right. \\
\left.\quad+\Phi^{2} \delta\left(\Phi^{2}\right)^{n-2}+\ldots+\left(\Phi^{2}\right)^{n-2} \delta \Phi^{2}+\left(\Phi^{2}\right)^{n-1} \delta\right] \\
=\sum_{n=0}^{\infty} \frac{1}{(2 n) !}\left[\left(\Phi^{2}\right)^{n}+n\left(\Phi^{2}\right)^{n-1}\left(-2 p^{2} \lambda\right)+\frac{1}{2} n(n-1)\left(\Phi^{2}\right)^{n-1}\left(-8 p^{2} \lambda\right)\right]
\end{aligned}
$$

where we have obtained the final line by commuting all $\delta$ 's through to the right, where they vanish. Combining the two terms proportional to $p^{2} \lambda$, we get

$$
\begin{aligned}
& \sum_{n=0}^{\infty} \frac{1}{(2 n) !}\left[\left(\Phi^{2}\right)^{n}-2 n(2 n-1)\left(\Phi^{2}\right)^{n-1} p^{2} \lambda\right] \\
& \quad=\sum_{n=0}^{\infty} \frac{1}{(2 n) !}\left(\Phi^{2}\right)^{n}\left(1-p^{2} \lambda\right)=\left(1-p^{2} \lambda\right) \cosh \Phi
\end{aligned}
$$

To show that Eq. (5.24) is equivalent to the exact result of Eq. (5.14), we must show that

$$
F(x)=\frac{1}{2 \pi} \int_{-\infty}^{\infty} d p e^{i p x}\left(e^{-p^{2} \lambda}-1+p^{2} \lambda\right)=\frac{1}{2 \pi} \int_{-\infty}^{\infty} d p e^{i p x} O\left(\lambda^{2} p^{4}\right) \leftrightarrow 0
$$

The most direct way to see this is by integration by parts,

$$
\int_{-\infty}^{\infty} d x x^{m} F(x)=\int_{-\infty}^{\infty} d p\left[\left(-i \frac{\partial}{\partial p}\right)^{m} \delta(p)\right] O\left(\lambda^{2} p^{4}\right)=0, \quad m \leqq 3 .
$$

An alternative way, which generalizes easily to the quaternionic case, is to use dimensional analysis: Scaling $p \rightarrow p / \lambda^{1 / 2}$ in Eq. (5.25), we see that $F(x)$ $=\lambda^{-1 / 2} f\left(x / \lambda^{1 / 2}\right)$, and so

$$
\int_{-\infty}^{\infty} d x x^{m} F(x) \propto \lambda^{m / 2}
$$


However, since the power series expansion of $F$ begins with order $\lambda^{2}$, the moments of Eq. (5.27) must vanish for $m \leqq 3$. We conclude, then, that

$$
\left\langle x, t\left|\exp \left(-i \Delta t p^{2} / 2 m\right)\right| 0, t\right\rangle \leftrightarrow \frac{1}{2 \pi} \int_{-\infty}^{\infty} d p \exp \left(i p x-p^{2} \lambda\right),
$$

which on integration yields Eq. (5.19a). Although the approximate derivation just described is more complicated than the exact analysis, it has the virtue of generalizing directly to the quaternionic case.

Let us now return to the quaternionic analysis which we left at Eq. (5.9). Using the coordinate space representation of $\widetilde{H}_{\text {kin }}$ given in Eq. (4.24), the left-hand side of Eq. (5.9) can be rewritten as

$$
\exp \left(-\Delta t \tilde{H}_{\text {kin }}\right)\langle\{\phi, \psi\}, t \mid\{0,0\}, t\rangle \exp \left(\Delta t \tilde{H}_{\text {kin }}\right) .
$$

The matrix element $\langle\{\phi, \psi\}, t \mid\{0,0\}, t\rangle$ is just the delta function $\delta(\phi, \psi)$ defined in Eq. (2.35), and by the arguments given above, the coordinate moments of Eq. (5.29) through second order are unchanged if we drop the quadratic and higher degree terms in $\widetilde{H}_{\text {kin }}$, giving

$$
\left\langle\{\phi, \psi\}, t\left|\exp \left(-\Delta t \tilde{H}_{\mathrm{kin}}\right)\right|\{0,0\}, t\right\rangle \leftrightarrow \delta(\phi, \psi)-\Delta t\left[\tilde{H}_{\mathrm{kin}}, \delta(\phi, \psi)\right] .
$$

Substituting the representation of $\delta(\phi, \psi)$ given in Eq. (2.42), and developing $\cosh \Phi$ in a power series expansion [with $\Phi$ henceforth defined by Eq. (2.42)], the righthand side of Eq. (5.30) becomes

$$
\left(\prod_{i=1}^{M} \int d u^{i} d \xi^{i}\right)(2 \pi)^{-4 M} \sum_{n=0}^{\infty} \frac{1}{(2 n) !}\left\{\left(\Phi^{2}\right)^{n}-\Delta t\left[\tilde{H}_{\mathrm{kin}},\left(\Phi^{2}\right)^{n}\right]\right\}
$$

We now introduce some definitions which facilitate the algebra of simplifying Eq. (5.31). To make the quaternion-dependence of $\Phi$ and $\widetilde{H}_{\text {kin }}$ explicit, we write

$$
\begin{gathered}
\Phi=\Phi_{1} e_{1}+\Phi_{2} e_{2}+\Phi_{3} e_{3}, \\
\Phi_{1}=\sum_{i=1}^{M}\left(\phi_{0}^{i} u_{1}^{i}-\phi_{1}^{i} u_{0}^{i}+\phi_{3}^{i} u_{2}^{i}-\phi_{2}^{i} u_{3}^{i}+\psi_{0}^{i} \xi_{1}^{i}-\psi_{1}^{i} \xi_{0}^{i}+\psi_{3}^{i} \xi_{2}^{i}-\psi_{2}^{i} \xi_{3}^{i}\right), \\
\Phi_{2}=\sum_{i=1}^{M}\left(\phi_{0}^{i} u_{2}^{i}-\phi_{2}^{i} u_{0}^{i}+\phi_{1}^{i} u_{3}^{i}-\phi_{3}^{i} u_{1}^{i}+\psi_{0}^{i} \xi_{2}^{i}-\psi_{2}^{i} \xi_{0}^{i}+\psi_{1}^{i} \xi_{3}^{i}-\psi_{3}^{i} \xi_{1}^{i}\right), \\
\Phi_{3}=\sum_{i=1}^{M}\left(\phi_{0}^{i} u_{3}^{i}-\phi_{3}^{i} u_{0}^{i}+\phi_{2}^{i} u_{1}^{i}-\phi_{1}^{i} u_{2}^{i}+\psi_{0}^{i} \xi_{3}^{i}-\psi_{3}^{i} \xi_{0}^{i}+\psi_{2}^{i} \xi_{1}^{i}-\psi_{1}^{i} \xi_{2}^{i}\right) ; \\
h_{1}=\frac{1}{3} \sum_{i=1}^{M}\left(\frac{\partial}{\partial \phi_{1}^{i}} \frac{\partial}{\partial \phi_{3}^{i}}-\frac{\partial}{\partial \phi_{0}^{i}} \frac{\partial}{\partial \phi_{2}^{i}}-\frac{\partial}{\partial \psi_{2}^{i}} \frac{\partial}{\psi_{3}^{i}}+\frac{\partial}{\partial \psi_{0}^{i}} \frac{\partial}{\partial \psi_{1}^{i}}\right), \\
h_{2}=\frac{1}{3} \sum_{i=1}^{M}\left(\frac{\partial}{\partial \phi_{0}^{i}} \frac{\partial}{\partial \phi_{1}^{i}}+\frac{\partial}{\partial \phi_{2}^{i}} \frac{\partial}{\partial \phi_{3}^{i}}-\frac{\partial}{\partial \psi_{3}^{i}} \frac{\partial}{\partial \psi_{1}^{i}}+\frac{\partial}{\partial \psi_{0}^{i}} \frac{\partial}{\partial \psi_{2}^{i}}\right), \\
h_{3}=\frac{1}{3} \sum_{i=1}^{M}\left[\frac{1}{2}\left(\frac{\partial}{\partial \phi_{0}^{i}} \frac{\partial}{\partial \phi_{0}^{i}}+\frac{\partial}{\partial \phi_{3}^{i}} \frac{\partial}{\partial \phi_{3}^{i}}-\frac{\partial}{\partial \phi_{1}^{i}} \frac{\partial}{\partial \phi_{1}^{i}}-\frac{\partial}{\partial \phi_{2}^{i}} \frac{\partial}{\partial \phi_{2}^{i}}\right)\right. \\
\left.-\frac{\partial}{\partial \psi_{1}^{i}} \frac{\partial}{\partial \psi_{2}^{i}}+\frac{\partial}{\partial \psi_{0}^{i}} \frac{\partial}{\partial \psi_{3}^{i}}\right] .
\end{gathered}
$$


A computation of the commutator $\left[h_{a}, \Phi_{b}\right]$ gives

$$
\begin{gathered}
{\left[h_{a}, \Phi_{b}\right]=\frac{1}{3} \delta_{a b} \kappa+\frac{1}{3} \varepsilon_{a b c} \lambda_{c},} \\
\kappa=\sum_{i=1}^{M}\left(u_{3}^{i} \frac{\partial}{\partial \phi_{0}^{i}}-u_{0}^{i} \frac{\partial}{\partial \phi_{3}^{i}}+u_{2}^{i} \frac{\partial}{\partial \phi_{1}^{i}}-u_{1}^{i} \frac{\partial}{\partial \phi_{2}^{i}}+\xi_{0}^{i} \frac{\partial}{\partial \psi_{0}^{i}}+\xi_{1}^{i} \frac{\partial}{\partial \psi_{1}^{i}}+\xi_{2}^{i} \frac{\partial}{\partial \psi_{2}^{i}}+\xi_{3}^{i} \frac{\partial}{\partial \psi_{3}^{i}}\right), \\
\lambda_{1}=\sum_{i=1}^{M}\left(u_{3}^{i} \frac{\partial}{\partial \phi_{1}^{i}}+u_{1}^{i} \frac{\partial}{\partial \phi_{3}^{i}}-u_{0}^{i} \frac{\partial}{\partial \phi_{2}^{i}}-u_{2}^{i} \frac{\partial}{\partial \phi_{0}^{i}}+\xi_{0}^{i} \frac{\partial}{\partial \psi_{1}^{i}}-\xi_{1}^{i} \frac{\partial}{\partial \psi_{0}^{i}}+\xi_{3}^{i} \frac{\partial}{\psi_{2}^{i}}-\xi_{2}^{i} \frac{\partial}{\partial \psi_{3}^{i}}\right), \\
\lambda_{2}=\sum_{i=1}^{M}\left(u_{1}^{i} \frac{\partial}{\partial \phi_{0}^{i}}+u_{0}^{i} \frac{\partial}{\partial \phi_{1}^{i}}+u_{2}^{i} \frac{\partial}{\partial \phi_{3}^{i}}+u_{3}^{i} \frac{\partial}{\partial \phi_{2}^{i}}+\xi_{0}^{i} \frac{\partial}{\partial \psi_{2}^{i}}-\xi_{2}^{i} \frac{\partial}{\psi_{0}^{i}}+\xi_{1}^{i} \frac{\partial}{\partial \psi_{3}^{i}}-\xi_{3}^{i} \frac{\partial}{\partial \psi_{1}^{i}}\right), \\
\lambda_{3}=\sum_{i=1}^{M}\left(u_{0}^{i} \frac{\partial}{\partial \phi_{0}^{i}}+u_{3}^{i} \frac{\partial}{\partial \phi_{3}^{i}}-u_{1}^{i} \frac{\partial}{\partial \phi_{1}^{i}}-u_{2}^{i} \frac{\partial}{\partial \phi_{2}^{i}}+\xi_{0}^{i} \frac{\partial}{\partial \psi_{3}^{i}}-\xi_{3}^{i} \frac{\partial}{\partial \psi_{0}^{i}}+\xi_{2}^{i} \frac{\partial}{\partial \psi_{1}^{i}}-\xi_{1}^{i} \frac{\partial}{\partial \psi_{2}^{i}}\right),
\end{gathered}
$$

and a computation of the commutators of $\kappa$ and $\lambda_{a}$ with $\Phi_{b}$ gives

$$
\begin{gathered}
{\left[\kappa, \Phi_{a}\right]=k_{a}, \quad\left[\lambda_{a}, \Phi_{b}\right]=\varepsilon_{a b c} k_{c}, \quad k_{1}=\sum_{i=1}^{M} 2\left(u_{1}^{i} u_{3}^{i}-u_{0}^{i} u_{2}^{i}+\xi_{0}^{i} \xi_{1}^{i}-\xi_{2}^{i} \xi_{3}^{i}\right),} \\
k_{2}=\sum_{i=1}^{M} 2\left(u_{2}^{i} u_{3}^{i}+u_{0}^{i} u_{1}^{i}+\xi_{0}^{i} \xi_{2}^{i}-\xi_{3}^{i} \xi_{1}^{i}\right), \\
k_{3}=\sum_{i=1}^{M} 2\left[\frac{1}{2}\left(u_{3}^{i} u_{3}^{i}+u_{0}^{i} u_{0}^{i}-u_{1}^{i} u_{1}^{i}-u_{2}^{i} u_{2}^{i}\right)+\xi_{0}^{i} \xi_{3}^{i}-\xi_{1}^{i} \xi_{2}^{i}\right] \\
k \equiv k_{1} e_{1}+k_{2} e_{2}+k_{3} e_{3}=\sum_{i=1}^{M}\left(\bar{u}^{i} e_{3} u^{i}+\bar{\xi}^{i} \xi^{i}\right) .
\end{gathered}
$$

In terms of these quantities, we find

$$
\left[-\tilde{H}_{\mathrm{kin}}, \Phi^{2}\right]=\frac{1}{3} k+\delta
$$

where $\delta$, given by

$$
\delta=-\frac{2}{3} \varepsilon_{a b c} \Phi_{b} \lambda_{c} e_{a}-\frac{2}{3} \Phi_{\kappa},
$$

has all differential operators ordered to the right, and has a commutator with $\Phi^{2}$ given by

$$
\left[\delta, \Phi^{2}\right]=-\frac{4}{3} \Phi k \Phi .
$$

Let us now apply these commutators to the evaluation of Eq. (5.31), taking note of the fact that

$$
\Phi^{2}=-\left(\Phi_{1}^{2}+\Phi_{2}^{2}+\Phi_{3}^{2}\right)
$$

is quaternion-real. On substituting Eq. (5.35), the sum in Eq. (5.31) becomes

$$
\begin{aligned}
\sum_{n=0}^{\infty} & \frac{1}{(2 n) !}\left[\left(\Phi^{2}\right)^{n}+n\left(\Phi^{2}\right)^{n-1} \Delta t \frac{1}{3} k+\Delta t \delta\left(\Phi^{2}\right)^{n-1}\right. \\
& \left.+\Phi^{2} \Delta t \delta\left(\Phi^{2}\right)^{n-2}+\ldots+\left(\Phi^{2}\right)^{n-2} \Delta t \delta \Phi^{2}+\left(\Phi^{2}\right)^{n-1} \Delta t \delta\right] \\
= & \sum_{n=0}^{\infty} \frac{1}{(2 n) !}\left[\left(\Phi^{2}\right)^{n}+n\left(\Phi^{2}\right)^{n-1} \Delta t \frac{1}{3} k+\frac{1}{2} n(n-1)\left(\Phi^{2}\right)^{n-2}\left(-\frac{4}{3} \Delta t \Phi k \Phi\right)\right] \\
= & \sum_{n=0}^{\infty} \frac{1}{(2 n) !}\left[\left(\Phi^{2}\right)^{n}+\frac{1}{2 n+1}\left(\Phi^{2}\right)^{n} \frac{\Delta t}{6} k+\frac{2}{2 n+1}\left(\Phi^{2}\right)^{n-1}\left(-\frac{1}{3} \Delta t \Phi k \Phi\right)\right]
\end{aligned}
$$


where to get the second line we have commuted all of the factors $\delta$ to the right, and to get the third line we have set $n \rightarrow n+1$ in the final two terms.

We now compare the power series in Eq. (5.39) with the corresponding power series obtained from the right-hand side of Eq. (5.9), which by the Gaussian integral formula of Eq. (2.16) has the integral representation

$$
\begin{aligned}
\left(4 \pi^{2}\right)^{-M} \exp \left[\sum_{i=1}^{M}\left(\bar{\phi}^{i} e_{3} \phi^{i}+\bar{\psi}^{i} \psi^{i}\right) / 2 \Delta t\right]= & \left(\prod_{i=1}^{M} \int d u^{i} d \xi^{i}\right)(2 \pi)^{-4 M} \exp \left(-\frac{1}{2} \Delta t k+\Phi\right) \\
= & \left(\prod_{i=1}^{M} \int d u^{i} d \xi^{i}\right)(2 \pi)^{-4 M} \frac{1}{2}\left[\exp \left(-\frac{1}{2} \Delta t k+\Phi\right)\right. \\
& \left.+\exp \left(-\frac{1}{2} \Delta t k-\Phi\right)\right]
\end{aligned}
$$

By a $\Delta t$-scaling argument analogous to that of Eq. (5.27), the moments of Eq. (5.40) up to second order are unaffected if we replace $\exp \left(-\frac{1}{2} \Delta t k \pm \Phi\right)$ by the first two terms in its power series expansion in $\Delta t$, giving after some algebra,

$$
\begin{aligned}
& \left(4 \pi^{2}\right)^{-M} \exp \left[\sum_{i=1}^{M}\left(\bar{\phi}^{i} e_{3} \phi^{i}+\bar{\psi}^{i} \psi^{i}\right) / 2 \Delta t\right] \leftrightarrow\left(\prod_{i=1}^{M} \int d u^{i} d \xi^{i}\right)(2 \pi)^{-4 M} \\
& \cdot \sum_{n=0}^{\infty} \frac{1}{(2 n) !}\left[\left(\Phi^{2}\right)^{n}+\frac{n+1}{2 n+1}\left(\Phi^{2}\right)^{n}\left(\frac{-\Delta t}{2} k\right)\right. \\
& \left.+\frac{n}{2 n+1}\left(\Phi^{2}\right)^{n-1}\left(\frac{-\Delta t}{2} \Phi k \Phi\right)\right] .
\end{aligned}
$$

Comparing Eq. (5.41) with Eqs. (5.30), (5.31), and (5.39), we see that to prove the desired result

$$
\left\langle\{\phi, \psi\}, t\left|\exp \left(-\Delta t \tilde{H}_{\mathrm{kin}}\right)\right|\{0,0\}, t\right\rangle \leftrightarrow\left(4 \pi^{2}\right)^{-M} \exp \left[\sum_{i=1}^{M}\left(\bar{\phi}^{i} e_{3} \phi^{i}+\bar{\psi}^{i} \psi^{i}\right) / 2 \Delta t\right]
$$

we must show that the integral of the difference between the power series in Eq. (5.41) and Eq. (5.39) vanishes, i.e.,

$$
0=\left(\prod_{i=1}^{M} \int d u^{i} d \xi^{i}\right)(2 \pi)^{-4 M} \sum_{n=0}^{\infty} \frac{1}{(2 n+1) !} \frac{1}{6}\left[(3 n+4)\left(\Phi^{2}\right)^{n} k+n\left(\Phi^{2}\right)^{n-1} \Phi k \Phi\right] .
$$

This will be true iff

$$
\begin{aligned}
0 & =\left(\prod_{i=1}^{M} \int d u^{i} d \xi^{i}\right)\left[(3 n+4)\left(\Phi^{2}\right)^{n} k+n\left(\Phi^{2}\right)^{n-1} \Phi k \Phi\right]=2(-1)^{n} e_{a} \Lambda_{a}, \\
\Lambda_{a} & =\left(\prod_{i=1}^{M} \int d u^{i} d \xi^{i}\right)\left[(n+2)\left(\Phi_{b}^{2}\right)^{n} k_{a}+n\left(\Phi_{b}^{2}\right)^{n-1} k_{c} \Phi_{c} \Phi_{a}\right],
\end{aligned}
$$

where to get the final line we have substituted $\Phi k=-k \Phi-2 k_{c} \Phi_{c}$.

We will prove that the quantities $\Lambda_{a}$ vanish by showing that the integrand in Eq. (5.44) is a total $u, \xi$ derivative. To do this we note that $\Phi$ is invariant under the interchanges $\phi^{i} \rightarrow-u^{i}, u^{i} \rightarrow \phi^{i}, \psi^{i} \rightarrow \xi^{i}, \xi^{i} \rightarrow \psi^{i}$, which suggests considering differen- 
tial operators $\kappa^{\prime}, \lambda_{a}^{\prime}$ obtained by these interchanges from $\kappa, \lambda_{a}$ above,

$$
\begin{aligned}
\kappa^{\prime}= & \sum_{i=1}^{M}\left(-\phi_{3}^{i} \frac{\partial}{\partial u_{0}^{i}}+\phi_{0}^{i} \frac{\partial}{\partial u_{3}^{i}}-\phi_{2}^{i} \frac{\partial}{\partial u_{1}^{i}}+\phi_{1}^{i} \frac{\partial}{\partial u_{2}^{i}}\right. \\
& \left.+\psi_{0}^{i} \frac{\partial}{\partial \xi_{0}^{i}}+\psi_{1}^{i} \frac{\partial}{\partial \xi_{1}^{i}}+\psi_{2}^{i} \frac{\partial}{\partial \xi_{2}^{i}}+\psi_{3}^{i} \frac{\partial}{\partial \xi_{3}^{i}}\right), \\
\lambda_{1}^{\prime}= & \sum_{i=1}^{M}\left(-\phi_{3}^{i} \frac{\partial}{\partial u_{1}^{i}}-\phi_{1}^{i} \frac{\partial}{\partial u_{3}^{i}}+\phi_{0}^{i} \frac{\partial}{\partial u_{2}^{i}}+\phi_{2}^{i} \frac{\partial}{\partial u_{0}^{i}}\right. \\
& \left.+\psi_{0}^{i} \frac{\partial}{\partial \xi_{1}^{i}}-\psi_{1}^{i} \frac{\partial}{\partial \xi_{0}^{i}}+\psi_{3}^{i} \frac{\partial}{\partial \xi_{2}^{i}}-\psi_{2}^{i} \frac{\partial}{\partial \xi_{3}^{i}}\right), \\
\lambda_{2}^{\prime}= & \sum_{i=1}^{M}\left(-\phi_{1}^{i} \frac{\partial}{\partial u_{0}^{i}}-\phi_{0}^{i} \frac{\partial}{\partial u_{1}^{i}}-\phi_{2}^{i} \frac{\partial}{\partial u_{3}^{i}}-\phi_{3}^{i} \frac{\partial}{\partial u_{2}^{i}}\right. \\
& \left.+\psi_{0}^{i} \frac{\partial}{\partial \xi_{2}^{i}}-\psi_{2}^{i} \frac{\partial}{\partial \xi_{0}^{i}}+\psi_{1}^{i} \frac{\partial}{\partial \xi_{3}^{i}}-\psi_{3}^{i} \frac{\partial}{\partial \xi_{1}^{i}}\right), \\
& \lambda_{3}^{\prime}=\sum_{i=1}\left(-\phi_{0}^{i} \frac{\partial}{\partial u_{0}^{i}}-\phi_{3}^{i} \frac{\partial}{\partial u_{3}^{i}}+\phi_{1}^{i} \frac{\partial}{\partial u_{1}^{i}}+\phi_{2}^{i} \frac{\partial}{\partial u_{2}^{i}}\right. \\
& \left.+\psi_{0}^{i} \frac{\partial}{\partial \xi_{3}^{i}}-\psi_{3}^{i} \frac{\partial}{\partial \xi_{0}^{i}}+\psi_{2}^{i} \frac{\partial}{\partial \xi_{1}^{i}}-\psi_{1}^{i} \frac{\partial}{\partial \xi_{2}^{i}}\right) .
\end{aligned}
$$

These have the following commutators with $\Phi_{a}$ and $k_{a}$,

$$
\left[\kappa^{\prime}, \Phi_{a}\right]=k_{a}^{\prime}, \quad\left[\lambda_{a}^{\prime}, \Phi_{b}\right]=\varepsilon_{a b c} k_{c}^{\prime}, \quad\left[\kappa^{\prime}, k_{a}\right]=2 \Phi_{a}, \quad\left[\lambda_{a}^{\prime}, k_{b}\right]=-2 \delta_{a b} \Psi+2 \varepsilon_{a b c} \Phi_{c},
$$

where

$$
\begin{aligned}
& \Psi=\sum_{i=1}^{M}\left(\phi_{0}^{i} u_{0}^{i}+\phi_{1}^{i} u_{1}^{i}+\phi_{2}^{i} u_{2}^{i}+\phi_{3}^{i} u_{3}^{i}+\psi_{0}^{i} \xi_{0}^{i}+\psi_{1}^{i} \xi_{1}^{i}+\psi_{2}^{i} \xi_{2}^{i}+\psi_{3}^{i} \xi_{3}^{i}\right), \\
& k_{1}^{\prime}=\sum_{i=1}^{M} 2\left(\phi_{1}^{i} \phi_{3}^{i}-\phi_{0}^{i} \phi_{2}^{i}+\psi_{0}^{i} \psi_{1}^{i}-\psi_{2}^{i} \psi_{3}^{i}\right) \\
& k_{2}^{\prime}=\sum_{i=1}^{M} 2\left(\phi_{2}^{i} \phi_{3}^{i}+\phi_{0}^{i} \phi_{1}^{i}+\psi_{0}^{i} \psi_{2}^{i}-\psi_{3}^{i} \psi_{1}^{i}\right), \\
& k_{3}^{\prime}=\sum_{i=1}^{M} 2\left[\frac{1}{2}\left(\phi_{3}^{i} \phi_{3}^{i}+\phi_{0}^{i} \phi_{0}^{i}-\phi_{1}^{i} \phi_{1}^{i}-\phi_{2}^{i} \phi_{2}^{i}\right)+\psi_{0}^{i} \psi_{3}^{i}-\psi_{1}^{i} \psi_{2}^{i}\right] .
\end{aligned}
$$

Since $\left(k_{1}^{\prime}\right)^{2}+\left(k_{2}^{\prime}\right)^{2}+\left(k_{3}^{\prime}\right)^{2} \neq 0$, to prove the vanishing of $\Lambda_{a}$ it suffices to show that

(i) $\quad 0=k_{a}^{\prime} \Lambda_{a}=\left(\prod_{i=1}^{M} \int d u^{i} d \xi^{i}\right)\left[(n+2)\left(\Phi_{b}^{2}\right)^{n} k_{a} k_{a}^{\prime}+n\left(\Phi_{b}^{2}\right)^{n-1} k_{c} \Phi_{c} k_{a}^{\prime} \Phi_{a}\right]$,

(ii) $0=\varepsilon_{a b d} \Lambda_{b} k_{d}^{\prime}=\left(\prod_{i=1}^{M} \int d u^{i} d \xi^{i}\right)\left[(n+2)\left(\Phi_{c}^{2}\right)^{n} \varepsilon_{a b d} k_{b} k_{d}^{\prime}+n\left(\Phi_{c}^{2}\right)^{n-1} k_{e} \Phi_{e} \varepsilon_{a b d} \Phi_{b} k_{d}^{\prime}\right]$. 
Now working with the commutators of Eq. (5.46), we find that

$$
\left[\kappa^{\prime},\left(\Phi_{c}^{2}\right)^{n} k_{b} \Phi_{b}\right]-\frac{1}{2} \varepsilon_{a b c}\left[\lambda_{a}^{\prime},\left(\Phi_{d}^{2}\right)^{n} k_{b} \Phi_{c}\right]=(n+2)\left(\Phi_{b}^{2}\right)^{n} k_{a} k_{a}^{\prime}+n\left(\Phi_{b}^{2}\right)^{n-1} k_{c} \Phi_{c} k_{a}^{\prime} \Phi_{a},
$$

which proves that the integrand of Eq. (5.48a) is a total $u, \xi$ derivative, and

$$
\begin{gathered}
{\left[\lambda_{b}^{\prime},\left(\Phi_{c}^{2}\right)^{n} k_{b} \Phi_{a}\right]-\left[\kappa^{\prime},\left(\Phi_{c}^{2}\right)^{n} \varepsilon_{a b e} k_{b} \Phi_{e}\right]=} \\
-6\left(\Phi_{c}^{2}\right)^{n} \Phi_{a} \Psi+2 n\left(\Phi_{c}^{2}\right)^{n-1} k_{e} \Phi_{e} \varepsilon_{a b d} \Phi_{b} k_{d}^{\prime} \\
-2(n+1)\left(\Phi_{c}^{2}\right)^{n} \varepsilon_{a b d} k_{b} k_{d}^{\prime}, \\
{\left[\lambda_{b}^{\prime},\left(\Phi_{c}^{2}\right)^{n} k_{a} \Phi_{b}\right]=-2\left(\Phi_{c}^{2}\right)^{n} \Phi_{a} \Psi,} \\
{\left[\lambda_{a}^{\prime},\left(\Phi_{c}^{2}\right)^{n} k_{b} \Phi_{b}\right]=-2\left(\Phi_{c}^{2}\right)^{n} \Phi_{a} \Psi+2 n\left(\Phi_{c}^{2}\right)^{n-1} k_{e} \Phi_{e} \varepsilon_{a b d} \Phi_{b} k_{d}^{\prime}+\left(\Phi_{c}^{2}\right)^{n} \varepsilon_{a b d} k_{b} k_{d}^{\prime},}
\end{gathered}
$$

which, since

$$
\operatorname{det}\left(\begin{array}{ccc}
-6 & 2 n & -2(n+1) \\
-2 & 0 & 0 \\
-2 & 2 n & 1
\end{array}\right)=4 n(2 n+3) \neq 0
$$

proves that the two terms in the integrand of Eq. (5.48b) are individually total $u, \xi$ derivatives. Thus our proof of Eq. (5.42) is complete.

\section{Projection onto Complex Quantum Mechanics ${ }^{6}$}

Since the complex number field $C\left(1, e_{3}\right)$ is a subspace of the quaternion field, we should be able to recover complex quantum mechanics (with a second order fermion wave operator) as a special case of the quaternionic quantum mechanics formulated in Sects. 3-5. We will explicitly verify this here, limiting ourselves for simplicity to the case $M=1$ in which a single bosonic and fermionic degree of freedom is present. We assume that the potential $\tilde{V}$ has the special form

$$
\tilde{V}=e_{3} V\left(\phi_{0}, \phi_{3}, \psi_{0}, \psi_{3}\right)
$$

with $V$ quaternion real. Then if the wave function $\Psi$ has, at any one time, the form

$$
\Psi=\Psi_{C}\left(\phi_{0}, \phi_{3}, \psi_{0}, \psi_{3}\right) \in C\left(1, e_{3}\right),
$$

this form will persist in time, and $\Psi_{C}$ will obey the complex Schrödinger equation

$$
\begin{gathered}
e_{3} \frac{\partial \Psi_{C}}{\partial t}=H \Psi_{C} \\
H=-\left.e_{3} \tilde{H}\right|_{\partial / \partial \phi_{1}=\partial / \partial \phi_{2}=\partial / \partial \psi_{1}=\partial / \partial \psi_{2}=0}=-\frac{1}{6}\left[\frac{\partial^{2}}{\left(\partial \phi_{0}\right)^{2}}+\frac{\partial^{2}}{\partial\left(\phi_{3}\right)^{2}}\right]-\frac{1}{3} \frac{\partial}{\partial \psi_{0}} \frac{\partial}{\partial \psi_{3}}+V .
\end{gathered}
$$

In the complex Lagrangian path integral formalism, the Hamiltonian of Eq. (6.3) corresponds to the transformation function for infinitesimal time interval $\Delta t$

$$
\begin{aligned}
& \left\langle\phi_{0}, \phi_{3}, \psi_{0}, \psi_{3}, t\left|\exp \left(-e_{3} \Delta t H\right)\right| \phi_{0}, \phi_{3}, \psi_{0}, \psi_{3}, t\right\rangle \\
& \quad=(2 \pi)^{-1} \exp \left[\frac{3}{2 \Delta t} e_{3}\left(\phi_{0}^{2}+\phi_{3}^{2}+2 \psi_{0} \psi_{3}\right)\right] \exp \left(-e_{3} \Delta t V\right) .
\end{aligned}
$$

${ }^{6}$ I wish to thank N. Seiberg for raising the question discussed in Sect. 6 
Consistency with the quaternionic path integral formalism of the preceding section requires that Eq. (6.4) be equivalent to the quaternionic transformation function of Eq. (5.9) when the unobserved components $\phi_{1,2}, \psi_{1,2}$ are integrated out. Taking into account Eq. (5.2), which allows us to factor away the potential dependence $\exp \left(-e_{3} \Delta t V\right)$, we thus require

$$
\begin{gathered}
\int d \phi_{1} d \phi_{2} d \psi_{1} d \psi_{2}\left(4 \pi^{2}\right)^{-1} \exp \left[\left(\bar{\phi} e_{3} \phi+\bar{\psi} \psi\right) / 2 \Delta t\right] \\
\leftrightarrow(2 \pi)^{-1} \exp \left[\frac{3}{2 \Delta t} e_{3}\left(\phi_{0}^{2}+\phi_{3}^{2}+2 \psi_{0} \psi_{3}\right)\right],
\end{gathered}
$$

with $\leftrightarrow$ the equivalence discussed in detail in Sect. 5. We will verify Eq. (6.5) in this section by explicitly computing the integral on the left-hand side, and then comparing the relevant coordinate moments of the left- and right-hand sides.

For convenience in the calculations which follow, we rescale $\phi \rightarrow \phi(2 \Delta t)^{1 / 2}$, $\psi \rightarrow \psi(2 \Delta t)^{1 / 2}$, and so (including a convergence factor) Eq. (6.5) becomes

$$
\begin{gathered}
\lim _{\varepsilon \rightarrow 0} \int d \phi_{1} d \phi_{2} d \psi_{1} d \psi_{2}(2 \pi)^{-1} \exp \left(\bar{\phi} e_{3} \phi+\bar{\psi} \psi-\varepsilon \bar{\phi} \phi\right) \leftrightarrow \exp \left[3 e_{3}\left(w+2 \psi_{0} \psi_{3}\right)\right], \\
w \equiv \phi_{0}^{2}+\phi_{3}^{2} .
\end{gathered}
$$

The first step in the evaluation of the left-hand side is to eliminate the quaternion dependence, using the method of Eqs. (2.21)-(2.24) above. Writing $\bar{\phi} e_{3} \phi+\bar{\psi} \psi$ $=\mathbf{v} \cdot \mathbf{e}$, we have

$$
\lim _{\varepsilon \rightarrow 0} \int d \phi_{1} d \phi_{2} d \psi_{1} d \psi_{2}(2 \pi)^{-1} \exp \left[-\varepsilon\left(\phi_{1}^{2}+\phi_{2}^{2}\right)\right]\left(\cos v+\frac{\mathbf{v} \cdot \mathbf{e}}{v} \sin v\right), \quad v=|\mathbf{v}|,
$$

and this must have the general form $a+b e_{3}$, giving the projected formulas

$$
\begin{aligned}
& a(v)=\lim _{\varepsilon \rightarrow 0} \int d \phi_{1} d \phi_{2} d \psi_{1} d \psi_{2}(2 \pi)^{-1} \exp \left[-\varepsilon\left(\phi_{1}^{2}+\phi_{2}^{2}\right)\right] \cos v, \\
& b(v)=\lim _{\varepsilon \rightarrow 0} \int d \phi_{1} d \phi_{2} d \psi_{1} d \psi_{2}(2 \pi)^{-1} \exp \left[-\varepsilon\left(\phi_{1}^{2}+\phi_{2}^{2}\right)\right]\left(v_{3} / v\right) \sin v=-\frac{\partial}{\partial v_{3}} a(v) .
\end{aligned}
$$

Hence it suffices to evaluate the integral $a(v)$, keeping the component $v_{3}$ distinguished until the end, or equivalently we evaluate

$$
a(v, \delta)=a(|\mathbf{v}+(0,0, \delta)|),
$$

to first order in $\delta$, in terms of which

$$
a(v)=a(v, 0), \quad b(v)=-\left.\frac{\partial}{\partial \delta} a(v, \delta)\right|_{\delta=0} .
$$

The remainder of the calculation consists of straightforward differentiations (to do the Grassmann integrals) and integrations, using the polar coordinates $\phi_{1}=R \cos \theta, \phi_{2}=R \sin \theta$. We skip the details, and proceed to the final answer,

$$
\begin{gathered}
\lim _{\varepsilon \rightarrow 0} \int d \phi_{1} d \phi_{2} d \psi_{1} d \psi_{2}(2 \pi)^{-1} \exp \left(\bar{\phi} e_{3} \phi+\bar{\psi} \psi-\varepsilon \bar{\phi} \phi\right) \\
=f_{1 R}(w)-\psi_{0} \psi_{3} f_{2 R}(w)+e_{3}\left[f_{1 I}(w)-\psi_{0} \psi_{3} f_{2 I}(w)\right], \\
w=\phi_{0}^{2}+\phi_{3}^{2}, \quad f_{1 R}(w)=\cos w+2 w \operatorname{si}(w), \\
f_{2 R}(w)=-2 \sin w-4 \operatorname{si}(w), \\
f_{1 I}(w)=\sin w+2 w \cos w+2 w^{2} \operatorname{si}(w), \quad f_{2 I}(w)=-6 \cos w-8 w \operatorname{si}(w),
\end{gathered}
$$


with $\operatorname{si}(w)$ the sine integral

$$
\operatorname{si}(w)=-\int_{w}^{\infty} d t \frac{\sin t}{t}
$$

Clearly, Eqs. (6.10) and (6.11) are not the same function as appears on the righthand side of Eq. (6.6),

$$
\exp \left[3 e_{3}\left(w+2 \psi_{0} \psi_{3}\right)\right]=\cos 3 w-6 \psi_{0} \psi_{3} \sin 3 w+e_{3}\left[\sin 3 w+6 \psi_{0} \psi_{3} \cos 3 w\right]
$$

However, straightforward integrations show that the moments through second order of Eqs. (6.10) and (6.12) agree,

$$
\begin{aligned}
& \lim _{\varepsilon \rightarrow 0} \int_{-\infty}^{\infty} d \phi_{0} d \phi_{3} \int d \psi_{0} d \psi_{3} \exp \left[-\varepsilon\left(\phi_{0}^{2}+\phi_{3}^{2}\right)\right]\left\{1, \phi_{0}^{2}+\phi_{3}^{2}, \psi_{0} \psi_{3}\right\} \\
& \cdot[\text { Eq. (6.10)-Eq. (6.12)] }=0 .
\end{aligned}
$$

[The needed integrals of si $(w)$ can all be obtained by differentiation of the integral

$$
\int_{0}^{\infty} d w \operatorname{si}(w) e^{-\varepsilon w}=-\frac{1}{\varepsilon} \tan ^{-1} \varepsilon,
$$

which serves as a moment-generating formula.] Hence Eqs. (6.12) and (6.10) are equivalent within a functional integral, thus proving Eq. (6.6). Beyond second order the moments of Eqs. (6.10) and (6.12) do not agree; for instance the fourth order moment difference

$$
\begin{gathered}
\lim _{\varepsilon \rightarrow 0} \int_{-\infty}^{\infty} d \phi_{0} d \phi_{3} \int d \psi_{0} d \psi_{3} \exp \left[-\varepsilon\left(\phi_{0}^{2}+\phi_{3}^{2}\right)\right]\left(\phi_{0}^{2}+\phi_{3}^{2}\right) \psi_{0} \psi_{3} \text { [Eq. (6.10)-Eq. (6.12)] } \\
\propto \lim _{\varepsilon \rightarrow 0} \int_{0}^{\infty} d w \exp (-\varepsilon w) w f_{1 R}(w)-\lim _{\varepsilon \rightarrow 0} \int_{0}^{\infty} d w \exp (-\varepsilon w) w \cos 3 w=\frac{1}{3}-\left(-\frac{1}{9}\right)
\end{gathered}
$$

is non-vanishing. We thus see in this section the same general feature excountered previously: In quaternionic quantum theory, all identities hold at the infinitesimal transformation level only! By contrast, in complex quantum theory, many of the formulas for infinitesimal transformations [such as Eq. (5.10) of the preceding section] extend to formulas valid for finite time steps as well.

\section{Breakdown of the Correspondence Principle}

In Sects. 4 and 5 we have seen that there are quaternionic analogs of the connection, in both directions, between complex quantum theory and the complex path integral. These, however, form only part of the apparatus of complex field theory; as shown in Fig. 1, a large part of the formalism of complex field theory relates to the correspondence principle, which establishes connections, in both directions, between the quantum theory and the corresponding system of classical field equations. ${ }^{7}$ Starting from the complex quantum theory, we have (i) the

\footnotetext{
${ }^{7}$ A brief discussion of the lack of a quaternionic analog of $S$-matrix theory is given in Sect. 10 below
} 


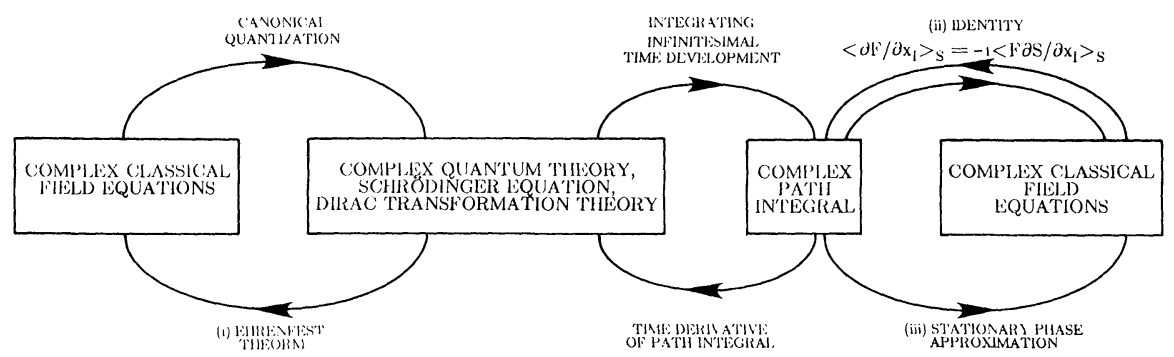

Fig. 1. The logical interrelations between complex classical field theory, complex quantum theory and the complex path integral

Ehrenfest theorem, which states that expectation values of the quantum equations of motion obey the corresponding classical field equations. The canonical quantization procedure can be viewed as an algorithm for inverting the Ehrenfest theorem, by reconstructing the complex quantum theory from its classical or correspondence limit. Starting from the path integral approach, the identity (ii)

$$
\left\langle\partial F / \partial x_{I}\right\rangle_{S}=-i\left\langle F \partial S / \partial x_{I}\right\rangle_{S},
$$

with $S$ the action, $F$ an arbitrary functional and $\partial / \partial x_{I}$ the derivative with respect to a canonical coordinate, yields [9] both the Ehrenfest theorem and the canonical commutation relations with appropriate choices of $F$. An alternative route from the path integral to the classical field equations is provided by (iii) the stationary phase approximation. We will show in this section that the avenues (i), (ii), and (iii) leading from complex quantum theory to the classical field equations all break down in quaternionic quantum mechanics.

We begin with (i), the Ehrenfest theorem. We start from the Schrödinger equation for $\Psi(\{\phi, \psi\}, t)$,

$$
\frac{\partial \Psi}{\partial t}=-\tilde{H} \Psi
$$

and for simplicity we omit the velocity potentials $A^{i}$ and $\beta^{i}$ so that

$$
\tilde{H}=\tilde{H}_{\text {kin }}+\tilde{V},
$$

with $\widetilde{H}_{\text {kin }}$ given by Eqs. (4.23)-(4.24). As a start, we must define our Hilbert space inner product, in terms of which we can construct operator expectations. A reconsideration of the analysis of Sects. 3-5 shows that we have already implicitly specified the inner product [see, for example, Eq. (3.4)] to be

$$
(\Phi, \Psi)=\left(\prod_{i=1}^{M} \int d \phi^{i} d \psi^{i}\right) \bar{\Phi}(\{\phi, \psi\}, t) \Psi(\{\phi, \psi\}, t) .
$$

As a check, we note that $\tilde{H}$ is anti-self-adjoint with respect to this inner product, and consequently the inner product is time-independent for states which evolve in time according to Eq. (7.2),

$$
\frac{\partial}{\partial t}(\Phi, \Psi)=\left(\frac{\partial \Phi}{\partial t}, \Psi\right)+\left(\Phi, \frac{\partial \Psi}{\partial t}\right)=-(\tilde{H} \Phi, \Psi)-(\Phi, \tilde{H} \Psi)=0 .
$$


We will see in Sect. 9 below that the quaternionic Hilbert space splits into two superselection sectors, in which $\Psi$ is respectively even and odd in the Grassmann variables. In the even sector the norm $(\Psi, \Psi)$ is real and we can define the expectation value $\langle\mathcal{O}\rangle$ of an observable $\mathcal{O}$ by

$$
\langle\mathcal{O}\rangle=(\Psi, \mathcal{O} \Psi) /(\Psi, \Psi)
$$

In the odd sector $(\Psi, \Psi)$ is quaternion-imaginary, and since the quaternion units do not commute the expressions $(\Psi, \Psi)^{-1}(\Psi, \mathcal{O} \Psi)$ and $(\Psi, \mathcal{O} \Psi)(\Psi, \Psi)^{-1}$ in general differ, and so in this sector we cannot define an unambiguous expectation value. Henceforth in this section we confine ourselves to the even sector, in which we consider the expectation of a bosonic variable

$$
\left\langle\phi_{a}^{j}\right\rangle=\left(\Psi, \phi_{a}^{j} \Psi\right) /(\Psi, \Psi) .
$$

To study the quaternionic version of the Ehrenfest theorem, we consider the time evolution of the expectation in Eq. (7.7),

$$
\frac{d}{d t}\left\langle\phi_{a}^{j}\right\rangle=\left(\Psi,\left(\tilde{H} \phi_{a}^{j}-\phi_{a}^{j} \tilde{H}\right) \Psi\right) /(\Psi, \Psi)
$$

To simplify the right-hand side of Eq. (7.8) we need the commutator $\left[\tilde{H}, \phi_{a}^{j}\right]$, which receives a contribution only from the term with $i=j$ in $\tilde{H}_{\text {kin }}$ of Eq. (4.23), giving

$$
\begin{gathered}
\frac{d}{d t}\left\langle\phi_{a}^{j}\right\rangle=\left\langle p_{a}^{j}\right\rangle, \\
p_{0}^{j}=\frac{1}{3} e_{1} \frac{\partial}{\partial \phi_{2}^{j}}-\frac{1}{3} e_{2} \frac{\partial}{\partial \phi_{1}^{j}}-\frac{1}{3} e_{3} \frac{\partial}{\partial \phi_{0}^{j}}, \\
p_{1}^{j}=-\frac{1}{3} e_{1} \frac{\partial}{\partial \phi_{3}^{j}}-\frac{1}{3} e_{2} \frac{\partial}{\partial \phi_{0}^{j}}+\frac{1}{3} e_{3} \frac{\partial}{\partial \phi_{1}^{j}}, \\
p_{2}^{j}=\frac{1}{3} e_{1} \frac{\partial}{\partial \phi_{0}^{j}}-\frac{1}{3} e_{2} \frac{\partial}{\partial \phi_{3}^{j}}+\frac{1}{3} e_{3} \frac{\partial}{\partial \phi_{2}^{j}}, \\
p_{3}^{j}=-\frac{1}{3} e_{1} \frac{\partial}{\partial \phi_{1}^{j}}-\frac{1}{3} e_{2} \frac{\partial}{\partial \phi_{2}^{j}}-\frac{1}{3} e_{3} \frac{\partial}{\partial \phi_{3}^{j}} .
\end{gathered}
$$

The quantities $p_{a}^{j}$ defined in Eq. (7.9) are self-adjoint, but since they are not quaternion real they have non-vanishing commutators with $\tilde{H}_{\text {kin }}$, and so $d\left\langle p_{a}^{j}\right\rangle / d t$ does not satisfy an analog of the Ehrenfest theorem. The $p_{a}^{j}$ 's also do not satisfy the standard canonical commutation relations, since

$$
\left[p_{a}^{j}, p_{b}^{j}\right] \neq 0 \quad a \neq b,
$$

and since

$$
\left[\phi_{a}^{i}, p_{b}^{j}\right]=\delta^{i j} C_{a b},
$$

with $C$ the non-diagonal matrix given by

$$
C=\frac{1}{3}\left[\begin{array}{cccc}
e_{3} & e_{2} & -e_{1} & 0 \\
e_{2} & -e_{3} & 0 & e_{1} \\
-e_{1} & 0 & -e_{3} & e_{2} \\
0 & e_{1} & e_{2} & e_{3}
\end{array}\right]
$$


with rows and columns in 0123 order. Clearly, the analogs in quaternionic quantum theory of the usual canonical momenta are the anti-Hermitian momenta $\tilde{p}_{a}^{i}$ defined by

$$
\tilde{p}_{a}^{i}=\frac{\partial}{\partial \phi_{a}^{i}} ;
$$

these satisfy, together with the $\phi_{a}^{i}$ s, the commutation relations

$$
\left[\phi_{a}^{i}, \phi_{b}^{j}\right]=\left[\tilde{p}_{a}^{i}, \tilde{p}_{b}^{j}\right]=0, \quad\left[\phi_{a}^{i}, \tilde{p}_{b}^{j}\right]=-\delta^{i j} \delta_{a b} .
$$

The $\tilde{p}_{a}^{i}$ s have a vanishing commutator with $\tilde{H}_{\text {kin }}$, and so satisfy an Ehrenfest theorem analog

$$
\frac{d}{d t}\left\langle\tilde{p}_{a}^{j}\right\rangle=\left\langle\left[\tilde{H}, \tilde{p}_{a}^{j}\right]\right\rangle=-\left\langle\frac{\partial}{\partial \phi_{a}^{j}} \tilde{V}\right\rangle .
$$

However, Eqs. (7.9) and (7.13) cannot be combined to give an analog of the Ehrenfest theorem for $d^{2}\left\langle\phi_{a}^{j}\right\rangle / d t^{2}$. The reason is that when we express the $p_{a}^{j}$ 's in terms of the $\tilde{p}_{a}^{j}$, we find

$$
\left\langle p_{a}^{j}\right\rangle=\left\langle-C_{a b} \tilde{p}_{b}^{j}\right\rangle,
$$

and since $C_{a b}$ does not commute with the quaternionic wave function $\Psi$, we cannot relate the right-hand side of Eq. (7.14) to the $\left\langle\tilde{p}_{b}^{j}\right\rangle$ 's. [In the complex case the $p_{a}^{j}$, $s$ and $\tilde{p}_{a}^{j}$ s are related by a factor of $i$, which commutes with everything, and so the standard Ehrenfest theorem relating $d^{2}\left\langle\phi_{a}^{j}\right\rangle / d t^{2}$ to $\left\langle-\partial V / \partial \phi_{a}^{j}\right\rangle$ immediately follows.]

We turn next to the path integral formalism, and examine the quaternionic analog of Feynman's identity of Eq. (7.1). Following Feynman, let $F(\{\phi(t), \psi(t)\}$, $\left.t_{0} \leqq t \leqq t_{N}\right)$ be a quaternion-real functional of the coordinates along the entire time path in Eq. (3.3), and define

$$
\begin{aligned}
\langle F\rangle_{\tilde{S}} & \equiv \int d[\phi] d[\psi] F(\{\phi(t), \psi(t)\}) T \exp \tilde{S}, \\
\tilde{S} & \equiv \int_{t_{0}}^{t_{N}} d u e_{a}(u) \tilde{L}_{a}(u) .
\end{aligned}
$$

Let $q_{I}$ be any individual component $\phi_{a}^{j}$ or $\psi_{a}^{j}$ at intermediate time $t_{I}$; since the integration measure is invariant under the change of integration variable $q_{I} \rightarrow q_{I}+\delta q_{I}$, we get the identity

$$
0=\int d[\phi] d[\psi]\left\{\frac{\partial}{\partial q_{I}} F T \exp \tilde{S}+F \frac{\partial}{\partial q_{I}}(T \exp \tilde{S})\right\} .
$$

The first line in Eq. (7.16) clearly gives $\left\langle\partial F / \partial q_{I}\right\rangle_{\tilde{S}}$, but despite the time-ordering the second line is not equal to

$$
\int d[\phi] d[\psi] F T\left(\frac{\partial}{\partial q_{I}} \tilde{S} \exp \tilde{S}\right)=\left\langle F \partial \tilde{S} / \partial q_{I}\right\rangle_{\tilde{S}}
$$

because even for fixed time $t_{I}$ the quaternion units $e_{a}\left(t_{I}\right)$ do not commute. Going back to the ordered product for which Eq. (7.15) is a shorthand, the $q_{I}$-dependence of the integrand resides entirely in the following factors ( $F$ can appear anywhere in 
the ordering since it is quaternion-real)

$$
e^{\Delta t \tilde{L}(I+1 / 2)} F e^{\Delta t \tilde{L}(I-1 / 2)},
$$

and we cannot derive Feynman's formula because

$$
\delta e^{\Delta t \tilde{L}(I+1 / 2)} \neq e^{\Delta t \tilde{L}(I+1 / 2)} \Delta t \delta \tilde{L}(I+1 / 2),
$$

and similarly for the right-hand factor. The best one can do is to get a formal result by using the equation, valid for the parametric variation of $\exp \mathcal{O}$ with $\mathcal{O}$ any operator,

$$
\frac{\partial}{\partial \lambda} e^{\mathscr{O}}=e^{\mathscr{U}} \int_{0}^{1} d s e^{-s \mathcal{O}} \frac{\partial \mathcal{O}}{\partial \lambda} e^{s \mathfrak{U}}=\int_{0}^{1} d s e^{s \mathfrak{U}} \frac{\partial \mathcal{O}}{\partial \lambda} e^{-s \mathfrak{U}} e^{\mathscr{U}} .
$$

Hence the quaternionic generalization of Eq. (7.1) states: $\langle F\rangle_{\tilde{S}}$ is replaced by 0 when the factor $F$ in the ordered product of Eq. (7.18) is replaced by

$$
\begin{aligned}
& \int_{0}^{1} d s\left\{e^{-s \Delta t \tilde{L}(I+1 / 2)} \Delta t \frac{\partial}{\partial q_{I}} \tilde{L}(I+1 / 2) e^{s \Delta t \tilde{L}(I+1 / 2)} F\right. \\
& \left.+\frac{\partial F}{\partial q_{I}}+F e^{s \Delta t \tilde{L}(I-1 / 2)} \Delta t \frac{\partial}{\partial q_{I}} \tilde{L}(I-1 / 2) e^{-s \Delta t \tilde{L}(I-1 / 2)}\right\} .
\end{aligned}
$$

We have not explored the consequences of the resulting formula, but since it differs in an essential way from Feynman's, the derivation given by Feynman of the Ehrenfest theorem and the canonical commutation relations breaks down in the quaternionic case. This is of course completely consistent with what we found from the Schrödinger equation analysis of Eqs. (7.2)-(7.14).

We turn finally to the stationary phase approximation, which is an alternative route by which classical equations are obtained as approximations to the path integral in the complex field theory case. In the quaternionic case, the stationary phase approximation breaks down for two reasons: (i) First, because of the timeordering in Eq. (3.3), the phase appearing in the functional integral is not the "classical" quaternionic action,

$$
T \exp \left[\int_{t_{0}}^{t_{N}} d t e_{a}(t) \tilde{L}_{a}(t)\right] \neq \exp \left[\tilde{S}_{\text {“classical" }}\right], \quad \tilde{S}_{\text {“classical }}=\int_{t_{0}}^{t_{N}} d t e_{a} \tilde{L}_{a}(t) .
$$

(ii) Second, even if (i) is ignored and the time-ordering in Eq. (7.22) is dropped, in general the variational problem $\delta \tilde{S}$ " classical" $=0$ has no solution. The reason for this is that $\widetilde{S}$ "classical" is stationary if

$$
\delta \int_{t_{0}}^{t_{N}} d t \widetilde{L}_{a}(t)=0, \quad a=1,2,3
$$

and the three real action components of Eq. (7.23) are not in general stationary on the same trajectories. ${ }^{8}$ Hence the quaternionic functional integral does not have a

\footnotetext{
${ }^{8}$ It is easily seen that $\tilde{L}$ has stationary action trajectories only in the special case when the variation of the potential $\tilde{V}$ has the form

$$
\delta \tilde{V}=\sum_{i}\left(\delta \bar{\phi}^{i} V_{i}^{R}+\delta \bar{\psi}^{i} \tau_{i}^{R}+V_{i}^{L} \delta \phi^{i}+\tau_{i}^{L} \delta \psi^{i}\right) .
$$

Thus the quaternionic oscillator $\tilde{L}=\frac{1}{2}\left(\bar{\phi} e_{3} \dot{\phi}-\omega^{2} \bar{\phi} e_{V} \phi\right)$, with $e_{V}$ an arbitrary unit quaternion, has stationary action trajectories, but $\tilde{L}=\frac{1}{2}\left(\bar{\phi} e_{3} \phi-\omega^{2} e_{V} \bar{\phi} \phi\right)$ does not, and neither does the quaternionic gauge field action $\widetilde{L}=\bar{F}_{\mu \nu} e_{3} F_{\mu v}, F_{\mu \nu}=\partial_{\mu} A_{v}-\partial_{v} A_{\mu}+\left[A_{\mu}, A_{v}\right]$ (with $\mu, v$ Lorentz indices)
} 


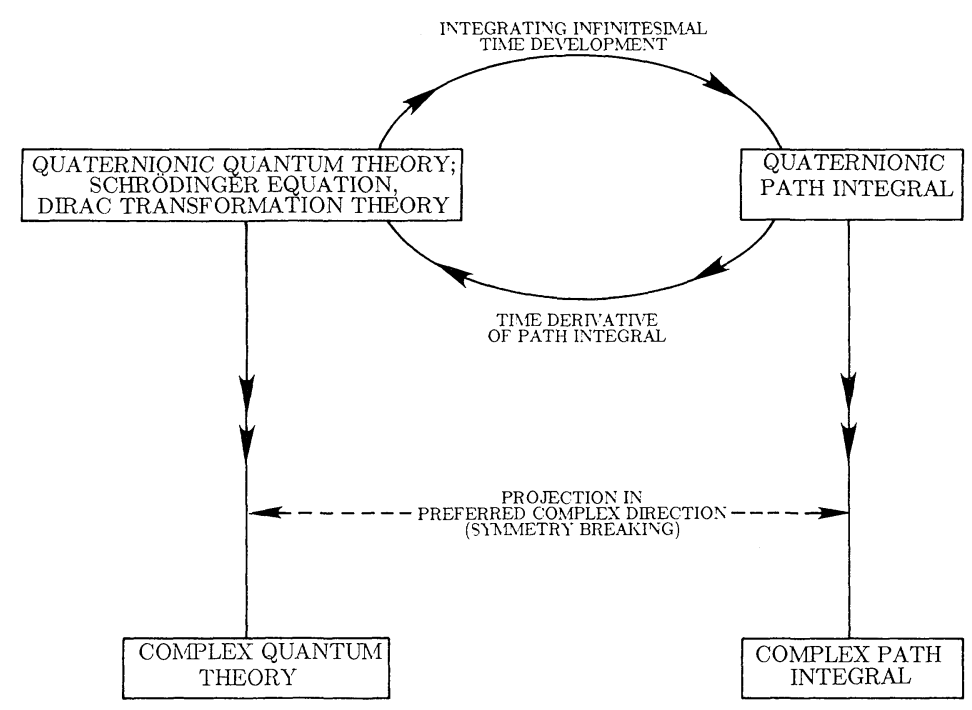

Fig. 2. The part of Fig. 1 which survives in the quaternionic case, and the connection (by symmetry breaking) to the complex case

semiclassical limit, again showing that the correspondence principle breaks down in quaternionic field theory.

To summarize, then, the only part of the complex field theory formalism of Fig. 1 which generalizes to the quaternionic case is that shown in Fig. 2. Quaternionic field theory can have a classical limit only through a projection (in which a preferred quaternionic unit is singled out) down to a complex field theory, which then has a complex classical limit of the usual kind diagrammed in Fig. 1.

\section{Boson State Structure}

In the formulation of quaternionic field theory given in Sects. 3-5, the underlying Hilbert space structure has remained implicit. The aim of this section and the next is to explicitly exhibit the structure of the Hilbert space for bosonic and fermionic quaternion degrees of freedom. For simplicity we restrict ourselves to $M=1$ (one bosonic and one fermionic degree of freedom), and start in this section with the bosonic sector. As discussed in Sect. 7, the scalar product implicit in Sects. 3-5 is, for bosons,

$$
\left(\Psi^{\prime}, \Psi\right)=\int d \phi_{0} d \phi_{1} d \phi_{2} d \phi_{3} \bar{\Psi}^{\prime}\left(\phi_{0}, \phi_{1}, \phi_{2}, \phi_{3}\right) \Psi\left(\phi_{0}, \phi_{1}, \phi_{2}, \phi_{3}\right) .
$$

This inner product satisfies the standard Hilbert space reality condition

$$
\overline{\left(\Psi^{\prime}, \Psi\right)}=\left(\Psi, \Psi^{\prime}\right)
$$

and gives a norm

$$
(\Psi, \Psi)=\int d \phi_{0} d \phi_{1} d \phi_{2} d \phi_{3}\left|\Psi\left(\phi_{0}, \phi_{1}, \phi_{2}, \phi_{3}\right)\right|^{2},
$$

which is positive definite, and vanishes only for $\Psi=0$. 
Let us now exhibit a complete set of bosonic states, which are simultaneously eigenstates of the boson kinetic operator $\widetilde{H}_{\text {kin }}=-(1 / 6) \bar{D}_{\phi} e_{3} D_{\phi}$. To do this let us start from the complex completeness relation

$$
\begin{gathered}
\frac{1}{(2 \pi)^{4}} \int d p e^{i\left(\lambda-\lambda^{\prime}\right)}=\delta\left(\phi-\phi^{\prime}\right)=\delta\left(\phi_{0}-\phi_{0}^{\prime}\right) \delta\left(\phi_{1}-\phi_{1}^{\prime}\right) \delta\left(\phi_{2}-\phi_{2}^{\prime}\right) \delta\left(\phi_{3}-\phi_{3}^{\prime}\right), \\
\lambda=p_{0} \phi_{0}+p_{1} \phi_{1}+p_{2} \phi_{2}+p_{3} \phi_{3}, \quad \lambda^{\prime}=p_{0} \phi_{0}^{\prime}+p_{1} \phi_{1}^{\prime}+p_{2} \phi_{2}^{\prime}+p_{3} \phi_{3}^{\prime}, \\
\int d p=\int d p_{0} d p_{1} d p_{2} d p_{3},
\end{gathered}
$$

which can be rewritten in terms of real functions as

$$
\frac{1}{(2 \pi)^{4}} \int d p\left(\cos \lambda \cos \lambda^{\prime}+\sin \lambda \sin \lambda^{\prime}\right)=\delta\left(\phi-\phi^{\prime}\right) \text {. }
$$

Defining the basis functions

$$
\Psi_{p e}(\phi)=\frac{1}{(2 \pi)^{2}} \cos \lambda q_{e}(p), \quad \Psi_{p o}(\phi)=\frac{1}{(2 \pi)^{2}} \sin \lambda q_{0}(p),
$$

with $q_{e, o}$ unit quaternions which can depend in an arbitrary way on $p$, the completeness relation takes the form

$$
\int d p\left[\Psi_{p e}\left(\phi^{\prime}\right) \bar{\Psi}_{p e}(\phi)+\Psi_{p o}\left(\phi^{\prime}\right) \bar{\Psi}_{p o}(\phi)\right]=\delta\left(\phi-\phi^{\prime}\right) .
$$

The bases $\Psi_{p e}$ and $\Psi_{p o}$ are eigenstates of $\tilde{H}_{\text {kin }}$,

$$
\begin{gathered}
\tilde{H}_{\mathrm{kin}} \Psi_{p e}=\Psi_{p e} \lambda_{e}(p), \quad \tilde{H}_{\mathrm{kin}} \Psi_{p o}=\Psi_{p o} \lambda_{o}(p), \\
\lambda_{e, o}(p)=\frac{1}{6} \bar{q}_{e, o}(p)\left[2 e_{1}\left(p_{1} p_{3}-p_{0} p_{2}\right)+2 e_{2}\left(p_{0} p_{1}+p_{2} p_{3}\right)\right. \\
\left.+e_{3}\left(p_{0}^{2}+p_{3}^{2}-p_{1}^{2}-p_{2}^{2}\right)\right] q_{e, o}(p) .
\end{gathered}
$$

Since

$$
4\left(p_{1} p_{3}-p_{0} p_{2}\right)^{2}+4\left(p_{0} p_{1}+p_{2} p_{3}\right)^{2}+\left(p_{0}^{2}+p_{3}^{2}-p_{1}^{2}-p_{2}^{2}\right)=\left(p_{0}^{2}+p_{1}^{2}+p_{2}^{2}+p_{3}^{2}\right)^{2},
$$

we can rewrite $\lambda_{e, o}(p)$ as

$$
\lambda_{e, o}(p)=\frac{1}{6}\left(p_{0}^{2}+p_{1}^{2}+p_{2}^{2}+p_{3}^{2}\right) e_{e, o}(p),
$$

with $e_{e, o}$ unit imaginary quaternions which can be given any orientation by an appropriate choice of $q_{e, o}(p)$.

In conclusion, we remark that ordering $\lambda_{e, o}(p)$ to the right in Eq. (8.7) was deliberate; the completeness relation for a general anti-self-adjoint operator $\mathcal{O}$ in a quaternionic Hilbert space takes the form

$$
\mathcal{O}=\sum_{\text {eigenvalues } o^{\prime}}\left|o^{\prime}\right\rangle o^{\prime}\left\langle o^{\prime}\right|
$$

which implies

$$
\mathcal{O}\left|o^{\prime \prime}\right\rangle=\sum_{\text {eigenvalues } o^{\prime}}\left|o^{\prime}\right\rangle o^{\prime}\left\langle o^{\prime} \mid o^{\prime \prime}\right\rangle=\left|o^{\prime \prime}\right\rangle o^{\prime \prime} .
$$

We cannot rewrite $\left|o^{\prime \prime}\right\rangle o^{\prime \prime}$ as $o^{\prime \prime}\left|o^{\prime \prime}\right\rangle$, since both $\left|o^{\prime \prime}\right\rangle$ and $o^{\prime \prime}$ are quaternion-valued, and hence non-commuting. In terms of eigenstates $\left|o^{\prime}\right\rangle$ the completeness relation 
reads

$$
1=\sum_{\text {eigenvalues } o^{\prime}}\left|o^{\prime}\right\rangle\left\langle o^{\prime}\right|
$$

To get Eq. (8.6) we let $\left|o^{\prime}\right\rangle=|p e, p o\rangle$ and take the $\left\langle\phi^{\prime}|\ldots| \phi\right\rangle$ matrix element of Eq. (8.11), giving

$$
\begin{gathered}
\left\langle\phi^{\prime}|1| \phi\right\rangle=\delta\left(\phi^{\prime}-\phi\right)=\sum_{p e, o}\left\langle\phi^{\prime} \mid p e, o\right\rangle\langle p e, o \mid \phi\rangle \\
=\int d p\left[\Psi_{p e}\left(\phi^{\prime}\right) \bar{\Psi}_{p e}(\phi)+\Psi_{p o}\left(\phi^{\prime}\right) \bar{\Psi}_{p o}(\phi)\right], \\
\Psi_{p e, o}(\phi)=\langle\phi \mid p e, o\rangle .
\end{gathered}
$$

Thus apart from care in factor ordering, the bosonic Hilbert space structure is entirely conventional.

\section{Fermion State Structure}

We turn our attention next to the state structure in the fermionic sector. Here we will find a number of unconventional features, arising both from the quaternionic structure and the use of a second order wave operator. As already noted in Sect. 7, the derivations of Sects. 3-5 implicitly assume the fermionic scalar product

$$
\left(\Psi^{\prime}, \Psi\right)=\int d \psi_{0} d \psi_{1} d \psi_{2} d \psi_{3} \bar{\Psi}^{\prime}\left(\psi_{0}, \psi_{1}, \psi_{2}, \psi_{3}\right) \Psi\left(\psi_{0}, \psi_{1}, \psi_{2}, \psi_{3}\right) .
$$

We cannot deduce from Eq. (9.1) that $\overline{\left(\Psi^{\prime}, \Psi\right)}=\left(\Psi, \Psi^{\prime}\right)$, because $\Psi$ and $\Psi^{\prime}$ are sums of monomials formed from anti-commuting Grassmann variables. To study the reality properties of Eq. (9.1), we employ the fact that the fermionic state space is finite dimensional, and so the general form of the wave function $\Psi$ is

$$
\begin{gathered}
\Psi=\Psi_{e}+\Psi_{o}, \\
\Psi_{e}=f+\psi_{0} \psi_{1} g_{1}+\psi_{0} \psi_{2} g_{2}+\psi_{0} \psi_{3} g_{3}+\psi_{2} \psi_{3} h_{1}+\psi_{3} \psi_{1} h_{2} \\
+\psi_{1} \psi_{2} h_{3}+\psi_{0} \psi_{1} \psi_{2} \psi_{3} k, \\
\Psi_{0}=\psi_{0} b+\psi_{1} c_{1}+\psi_{2} c_{2}+\psi_{3} c_{3}+\psi_{0} \psi_{2} \psi_{3} d_{1}+\psi_{0} \psi_{3} \psi_{1} d_{2} \\
+\psi_{0} \psi_{1} \psi_{2} d_{3}+\psi_{1} \psi_{2} \psi_{3} e,
\end{gathered}
$$

with $b, f, c_{1}, g_{1}, \ldots$ general quaternion coefficients. To determine the quaternion conjugation properties of Grassmann monomials, we use the following simple lemma: For any real or Grassmann quaternion $Q, Q=\bar{Q}$ iff $Q e_{f}=e_{f} Q, f=1,2,3$. [The proof follows immediately from Eq. (1.2).] Since we have defined the Grassmann components $\psi_{a}$ to be quaternion-real, we have $\psi_{a}=\bar{\psi}_{a}$, and the lemma then implies $\psi_{a} e_{f}=e_{f} \psi_{a}, f=1,2,3$. This in turn implies that any monomial formed from the $\psi_{a}$ 's commutes with $e_{f}, f=1,2,3$, and hence by the lemma is quaternion real. (Although the argument is elementary, we have formalized it in this way to stress the distinction from the complex case, where $i z=z i$ does not imply $z=\bar{z}$.)

Since we have just shown that the Grassmann monomials obey

$$
\bar{\psi}_{a}=\psi_{a}, \quad \overline{\psi_{a} \psi_{b}}=\psi_{a} \psi_{b}, \quad \overline{\psi_{a} \psi_{b} \psi_{c}}=\psi_{a} \psi_{b} \psi_{c}, \quad \overline{\psi_{0} \psi_{1} \psi_{2} \psi_{3}}=\psi_{0} \psi_{1} \psi_{2} \psi_{3},
$$


the adjoint wave function appearing in Eq. (9.1) has the form

$$
\begin{gathered}
\bar{\Psi}^{\prime}=\bar{\Psi}_{e}^{\prime}+\bar{\Psi}_{o}^{\prime}, \\
\bar{\Psi}_{e}^{\prime}=\bar{f}^{\prime}+\bar{g}_{1}^{\prime} \psi_{0} \psi_{1}+\bar{g}_{2}^{\prime} \psi_{0} \psi_{2}+\bar{g}_{3}^{\prime} \psi_{0} \psi_{3}+\bar{h}_{1}^{\prime} \psi_{2} \psi_{3}+{\overline{h_{2}^{\prime}}}_{2} \psi_{3} \psi_{1} \\
+\bar{h}_{3}^{\prime} \psi_{1} \psi_{2}+\bar{k}^{\prime} \psi_{0} \psi_{1} \psi_{2} \psi_{3}, \\
\bar{\Psi}_{o}^{\prime}=\bar{b}^{\prime} \psi_{0}+\bar{c}_{1}^{\prime} \psi_{1}+\bar{c}_{2}^{\prime} \psi_{2}+\bar{c}_{3}^{\prime} \psi_{3}+\bar{d}_{1}^{\prime} \psi_{0} \psi_{2} \psi_{3}+\bar{d}_{2}^{\prime} \psi_{0} \psi_{3} \psi_{1} \\
+\bar{d}_{3}^{\prime} \psi_{0} \psi_{1} \psi_{2}+\bar{e}^{\prime} \psi_{1} \psi_{2} \psi_{3},
\end{gathered}
$$

and the Grassmann monomials can be freely commuted through the quaternion coefficients in Eqs. (9.2) and (9.4). Substituting Eqs. (9.2) and (9.4) into Eq. (9.1), a short calculation shows that

$$
\begin{gathered}
\left(\Psi^{\prime}, \Psi\right)=\left(\Psi_{e}^{\prime}, \Psi_{e}\right)+\left(\Psi_{o}^{\prime}, \Psi_{o}\right), \\
\left(\Psi_{e}^{\prime}, \Psi_{e}\right)=\bar{f}^{\prime} k+\bar{k}^{\prime} f+\bar{g}_{1}^{\prime} h_{1}+\bar{h}_{1}^{\prime} g_{1}+\bar{g}_{2}^{\prime} h_{2}+\bar{h}_{2}^{\prime} g_{2}+\bar{g}_{3}^{\prime} h_{3}+\bar{h}_{3}^{\prime} g_{3}, \\
\left(\Psi_{o}^{\prime}, \Psi_{o}\right)=\bar{b}^{\prime} e-\bar{e}^{\prime} b-\bar{c}_{1}^{\prime} d_{1}+\bar{d}_{1}^{\prime} c_{1}-\bar{c}_{2}^{\prime} d_{2}+\bar{d}_{2}^{\prime} c_{2}-\bar{c}_{3}^{\prime} d_{3}+\bar{d}_{3}^{\prime} c_{3} .
\end{gathered}
$$

We see that the fermion state space splits into two orthogonal sectors; in the sector spanned by even Grassmann monomials, the inner product of Eq. (9.1) satisfies the reality condition

$$
\overline{\left(\Psi_{e}^{\prime}, \Psi_{e}\right)}=\left(\Psi_{e}, \Psi_{e}^{\prime}\right)
$$

while in the sector spanned by the odd Grassmann monomials, the inner product satisfies an anti-reality condition

$$
\overline{\left(\Psi_{o}^{\prime}, \Psi_{o}\right)}=-\left(\Psi_{o}, \Psi_{o}^{\prime}\right)
$$

Equations (9.6) imply that the norms of even and odd monomials satisfy ${ }^{9}$

$$
\begin{aligned}
& \overline{\left(\Psi_{e}, \Psi_{e}\right)}=\left(\Psi_{e}, \Psi_{e}\right), \\
& \overline{\left(\Psi_{o}, \Psi_{o}\right)}=-\left(\Psi_{o}, \Psi_{o}\right),
\end{aligned}
$$

and are quaternion-real and quaternion-imaginary, respectively! Furthermore, there is no way of redefining the inner product, by multiplication by an imaginary unit, so as to make $\left(\Psi_{o}, \Psi_{o}\right)$ manifest real. We conclude that in quaternionic quantum theory, the physical Hilbert space must be constructed from the states $\Psi_{e}$ only. This restriction is consistent if the Lagrangian $\tilde{L}$ is even in the Grassmann variables, since then only even states can be prepared from the vacuum by any physical processes.

Before continuing our discussion, let us pause to examine the complex analog of Eqs. (9.1)-(9.7). In the complex case we have

$$
\begin{gathered}
\left(\Psi^{\prime}, \Psi\right)=\int d \psi_{1} d \psi_{0} \bar{\Psi}^{\prime}\left(\psi_{0}, \psi_{1}\right) \Psi\left(\psi_{0}, \psi_{1}\right), \\
\Psi=\Psi_{e}+\Psi_{o}, \quad \Psi_{e}=f+\psi_{0} \psi_{1} g, \quad \Psi_{o}=\psi_{0} b+\psi_{1} c .
\end{gathered}
$$

\footnotetext{
${ }^{9}$ These statements generalize readily to the case when the number of quaternionic fermion degrees of freedom is any $M>1$. The product of two monomials of degrees $d_{1}, d_{2}$ contributes to the inner product only if $d_{1}+d_{2}=4 M$; hence $d_{1}$ and $d_{2}$ are either both odd or both even. The interchange of the two monomials results in a factor $(-1)^{d_{1} d_{2}}=-1(+1)$ in the odd (even) cases respectively, resulting in an inner product which is quaternion-imaginary (real)
} 
If we conjugate $\psi_{0} \psi_{1}$ as in Eq. (9.3), so that $\overline{\psi_{0} \psi_{1}}=\psi_{0} \psi_{1}$, then we find

$$
\bar{\Psi}^{\prime}=\bar{\Psi}_{e}^{\prime}+\bar{\Psi}_{o}^{\prime}, \quad \bar{\Psi}_{e}^{\prime}=\bar{f}^{\prime}+\bar{g}^{\prime} \psi_{0} \psi_{1}, \quad \bar{\Psi}_{o}^{\prime}=\bar{b}^{\prime} \psi_{0}+\bar{c}^{\prime} \psi_{1} .
$$

This gives for the inner product

$$
\begin{aligned}
& \left(\Psi^{\prime}, \Psi\right)=\left(\Psi_{e}^{\prime}, \Psi_{e}\right)+\left(\Psi_{o}^{\prime}, \Psi_{o}\right), \\
& \left(\Psi_{e}^{\prime}, \Psi_{e}\right)=\bar{f}^{\prime} g+\bar{g}^{\prime} f \Rightarrow \overline{\left(\Psi_{e}^{\prime}, \Psi_{e}\right)}=\left(\Psi_{e}, \Psi_{e}^{\prime}\right), \\
& \left(\Psi_{o}^{\prime}, \Psi_{o}\right)=-\bar{c}^{\prime} b+\bar{b}^{\prime} c \Rightarrow \overline{\left(\Psi_{o}^{\prime}, \Psi_{o}\right)}=-\left(\Psi_{o}, \Psi_{o}^{\prime}\right),
\end{aligned}
$$

and so again we encounter an imaginary norm. However, in the complex case the lemma used to derive Eq. (9.3) does not hold, and so it is perfectly consistent to define the conjugation of $\psi_{0} \psi_{1}$ by $\overline{\psi_{0} \psi_{1}}=\psi_{1} \psi_{0}=-\psi_{0} \psi_{1}$. The second line in Eq. (9.9) is then changed to

$$
\bar{\Psi}_{e}^{\prime}=\bar{f}^{\prime}-\bar{g}^{\prime} \psi_{0} \psi_{1},
$$

and the second line of Eq. (9.10) becomes

$$
\left(\Psi_{e}^{\prime}, \Psi_{e}\right)=\bar{f}^{\prime} g-\bar{g}^{\prime} f \Rightarrow \overline{\left(\Psi_{e}^{\prime}, \Psi_{e}\right)}=-\left(\Psi_{e}, \Psi_{e}^{\prime}\right) .
$$

The norms in the even and odd sectors are now both imaginary, but we can make them both real by multiplying by $i$, effectively making the inner product

$$
\begin{gathered}
\left(\Psi^{\prime}, \Psi\right)=i \int d \psi_{1} d \psi_{0} \bar{\Psi}^{\prime}\left(\psi_{0}, \psi_{1}\right) \Psi\left(\psi_{0}, \psi_{1}\right), \\
\left(\Psi_{e}^{\prime}, \Psi_{e}\right)=i\left(\overline{f^{\prime} g}-\bar{g}^{\prime} f\right)=\overline{\left(\Psi_{e}, \Psi_{e}^{\prime}\right)} \text { with } \overline{\psi_{0} \psi_{1}}=\psi_{1} \psi_{0}, \\
\left(\Psi_{o}^{\prime}, \Psi_{o}\right)=i\left(\overline{b^{\prime} c}-\bar{c}^{\prime} b\right)=\overline{\left(\Psi_{o}, \Psi_{o}^{\prime}\right)}
\end{gathered}
$$

The conventions of Eq. (9.11c) are the standard ones used in complex field theory. The same physics is in fact obtained irrespective of whether the standard conventions of Eq. (9.11c), or the quaternionic conventions of Eqs. (9.1)-(9.7), are used. To see this, we note that (i) in theories of physical interest, fermion number is governed by a superselection rule, and so the fermionic Grassmann variables always appear in the action in the combination $\widetilde{\Psi} \Gamma \psi$, with $\Gamma$ some matrix and $\psi$ a column vector with components of the form $\psi_{0}+i \psi_{1}$; (ii) For such combinations, the two conventions are related by

$$
(\bar{\psi} \Gamma \psi)_{\text {standard convention }}= \pm i(\bar{\psi} \Gamma \psi)_{\text {quaternionic convention }}
$$

since Eq. (9.12) is the condition for them to have the same Hermiticity properties. However, the factor $\pm i$ can be absorbed in the Grassmann integration measure in the functional integral formulation [13] of the $S$-matrix, giving the same $S$-matrix up to an unobservable overall phase. With these remarks in mind, in the remainder of this section we will consistently use the quaternionic conventions, even when discussing the complex case as an illustration of our formalism.

Since the quaternionic Grassmann vector space is an indefinite metric Hilbert space, let us next discuss some general properties of the indefinite metric formalism. Let $f_{i}(\psi) \equiv\langle\psi \mid i\rangle$ be a complete set of polynomials formed from the Grassmann variable $\psi$, orthogonal (but not orthonormal!) with respect to the inner product $\int d \psi \bar{f}^{\prime} f$. We thus have

$$
\int d \psi \overline{f_{i^{\prime}}}(\psi) f_{i}(\psi)=c_{i} \delta_{i i^{\prime}},
$$


with $c_{i} \neq 0$ a state normalization which in general can be quaternion valued. In the transformation theory notation, Eq. (9.13a) can be rewritten as

$$
\left\langle i^{\prime} \mid i\right\rangle=\int d \psi\left\langle i^{\prime} \mid \psi\right\rangle\langle\psi \mid i\rangle=c_{i} \delta_{i i^{\prime}},
$$

which implies the representation of unity

$$
1=\int d \psi|\psi\rangle\langle\psi| .
$$

Equation (9.14a), or rather its generalization to include bosons

$$
1=\int d \phi d \psi|\phi, \psi\rangle\langle\phi, \psi|,
$$

is what we used in constructing path integrals in Sects. 3 and 4, and we see that these representations of unity are independent of the normalization of the basis. The $c_{i}$ 's $d o$ enter explicitly when we construct $\delta\left(\psi^{\prime}-\psi\right)$ from the polynomials $f_{i}(\psi)$, since we have the identity

$$
\delta\left(\psi^{\prime}-\psi\right)=\sum_{i} f_{i}\left(\psi^{\prime}\right) \frac{1}{c_{i}} \bar{f}_{i}(\psi) .
$$

To prove Eq. (9.15), we let

$$
F\left(\psi^{\prime}, \psi\right)=\sum_{i} f_{i}\left(\psi^{\prime}\right) \frac{1}{c_{i}} \bar{f}_{i}(\psi),
$$

and for any index $j$ we consider the integral

$$
\int d \psi F\left(\psi^{\prime}, \psi\right) f_{j}(\psi)=\sum_{i} f_{i}\left(\psi^{\prime}\right) \frac{1}{c_{i}} \int d \psi \bar{f}_{i}(\psi) f_{j}(\psi)=\sum_{i} f_{i}\left(\psi^{\prime}\right) \frac{1}{c_{i}} \delta_{i j} c_{i}=f_{j}\left(\psi^{\prime}\right) .
$$

Since the $f_{j}(\psi)$ form a complete basis set, Eq. (9.16b) implies that

$$
F\left(\psi^{\prime}, \psi\right)=\delta\left(\psi^{\prime}-\psi\right)=\left\langle\psi^{\prime}|1| \psi\right\rangle=\left(\psi^{\prime}-\psi\right)_{0}\left(\psi^{\prime}-\psi\right)_{1}\left(\psi^{\prime}-\psi\right)_{2}\left(\psi^{\prime}-\psi\right)_{3}
$$

in the quaternionic case, and

$$
F\left(\psi^{\prime}, \psi\right)=\delta\left(\psi^{\prime}-\psi\right)=\left\langle\psi^{\prime}|1| \psi\right\rangle=\left(\psi^{\prime}-\psi\right)_{0}\left(\psi^{\prime}-\psi\right)_{1}
$$

if we specialize to the complex case. A second place where the $c_{i}$ 's enter explicitly is if we try to find linear combinations of the polynomials $f_{i}(\psi)$ which are eigenstates of the anti-Hermitian Hamiltonian operator $\tilde{H}$. Let us consider the matrix representation of $\tilde{H}$,

$$
\left\langle i^{\prime}|\tilde{H}| i\right\rangle=\int d \psi \overline{f_{i^{\prime}}}(\psi) \tilde{H} f_{i}(\psi),
$$

which defines a finite-dimensional quaternion-anti-self-adjoint matrix $\left\langle i^{\prime}|\tilde{H}| i\right\rangle$. The general spectral theorem quoted in Sect. 2 guarantees the existence of a unitary matrix which diagonalizes $\left\langle i^{\prime}|\tilde{H}| i\right\rangle$,

$$
\sum_{i^{\prime} i^{\prime}} \bar{U}_{i^{\prime} m}\left\langle i^{\prime}|\tilde{H}| i\right\rangle U_{i n}=\delta_{m n} e_{3} d_{n} .
$$

The left-hand side of Eq. (9.19) can be rewritten as

$$
\int d \psi \overline{\sum_{i^{\prime}} f_{i^{\prime}}(\psi) U_{i^{\prime} m}} \tilde{H} \sum_{i} f_{i}(\psi) U_{i n},
$$


but this does not mean that

$$
\tilde{H} \sum_{i} f_{i}(\psi) U_{i n}=\sum_{i} f_{i}(\psi) U_{i n} e_{3} d_{n}
$$

since Eq. (9.21) would imply

$$
\begin{aligned}
\delta_{m n} e_{3} d_{n} & =\int d \psi \overline{\sum_{i^{\prime}} f_{i^{\prime}}(\psi) U_{i^{\prime} m}} \sum_{i} f_{i}(\psi) U_{i n} e_{3} d_{n}=\sum_{i, i^{\prime}} \bar{U}_{i^{\prime} m} \int d \psi \bar{f}_{i^{\prime}}(\psi) f_{i}(\psi) U_{i n} e_{3} d_{n} \\
& =\sum_{i} \bar{U}_{i m} c_{i} U_{i n} e_{3} d_{n}
\end{aligned}
$$

that is

$$
\delta_{m n}=\sum_{i} \bar{U}_{i m} c_{i} U_{i n}
$$

Equation (9.22b) is true for $c_{i}=1$ (the usual case of orthonormalized states), but fails for general $c_{i}$, and so the diagonal transformation of $\left\langle i^{\prime}|\tilde{H}| i\right\rangle$ does not in general lead to polynomial functions of $\psi$ which diagonalize $\tilde{H}$.

Let us now apply this general formalism, first to the complex, and then to the quaternionic, case. In the complex case we take as the orthogonal basis functions

$$
f_{e \pm}(\psi)=\frac{1}{\sqrt{2}}\left(1 \pm \psi_{0} \psi_{1}\right), \quad f_{o \pm}(\psi)=\frac{1}{\sqrt{2}}\left(\psi_{0 \mp} i \psi_{1}\right)
$$

which have the respective norms

$$
\int d \psi_{1} d \psi_{0} \bar{f}_{e \pm}(\psi) f_{e \pm}(\psi)= \pm 1, \quad \int d \psi_{1} d \psi_{0} \bar{f}_{o \pm}(\psi) f_{o \pm}(\psi)=\mp i
$$

Hence from Eqs. (9.15) and (9.17b) we expect

$$
\begin{aligned}
\delta\left(\psi^{\prime}-\psi\right)= & \left(\psi^{\prime}-\psi\right)_{0}\left(\psi^{\prime}-\psi\right)_{1}=f_{e+}\left(\psi^{\prime}\right) \bar{f}_{e+}(\psi)-f_{e-}\left(\psi^{\prime}\right) \bar{f}_{e-}(\psi) \\
& +f_{o+}\left(\psi^{\prime}\right) i \bar{f}_{o+}(\psi)-f_{o-}\left(\psi^{\prime}\right) i \bar{f}_{o-}(\psi),
\end{aligned}
$$

and substitution of Eq. (9.23a) shows that this is indeed the case. To illustrate the remarks of Eqs. (9.18)-(9.22), let us consider the effect of the second order wave operator [cf. Eq. (6.3)]

$$
\tilde{H}=i c \frac{\partial}{\partial \psi_{0}} \frac{\partial}{\partial \psi_{1}},
$$

looking first at the even sector. The matrix $\langle e \pm|\widetilde{H}| e \pm\rangle$ is

$$
-\frac{i c}{2}\left(\begin{array}{rr}
1 & -1 \\
-1 & 1
\end{array}\right)
$$

and is anti-Hermitian, with eigenstates $\left(\begin{array}{l}1 \\ 1\end{array}\right)$ and $\left(\begin{array}{r}1 \\ -1\end{array}\right)$. However, the linear combinations $f_{e+} \pm f_{e-}$ are not both eigenstates of $\tilde{H}$; we instead have

$$
\tilde{H}\left(f_{e+}+f_{e^{-}}\right)=0, \quad \tilde{H}\left(f_{e^{+}}-f_{e^{-}}\right)=-i c\left(f_{e^{+}}+f_{e^{-}}\right),
$$

and clearly there is no multiple of $f_{e+}+f_{e_{-}}$which, when added to $f_{e_{+}}-f_{e_{-}}$, makes the latter an $\tilde{H}$ eigenstate. The reason for this is that the norms of $f_{e \pm}$ are opposite 
in sign, and so the even sector by itself is an indefinite metric space containing zero norm states $f_{e+} \pm f_{e_{-}}$. In the odd sector $\tilde{H}$ vanishes identically, but the odd sector is also an indefinite metric Hilbert space, with zero norm states $f_{n+} \pm f_{o-}$. Since the action of the time evolution operator $\exp (-\tilde{H} t)$ on $f_{e+} \pm f_{e-}, f_{o+} \pm f_{o-}$ is

$$
\begin{aligned}
& \exp (-\tilde{H} t)\left(f_{e+}+f_{e^{-}}\right)=\left(f_{e^{+}}+f_{e^{-}}\right) \\
& \exp (-\tilde{H} t)\left(f_{e+}-f_{e^{-}}\right)=f_{e_{+}}-f_{e^{-}}+\operatorname{ict}\left(f_{e^{+}}+f_{e^{-}}\right), \\
& \exp (-\tilde{H} t)\left(f_{o+} \pm f_{o^{-}}\right)=f_{o_{+}} \pm f_{o^{-}},
\end{aligned}
$$

all states are propagated forward in time and the + and - states in the even sector are mixed. Hence the use of a second order fermionic wave operator is necessarily associated with an indefinite metric state structure.

To achieve a definite metric fermionic Hilbert space with no zero norm states, we must eliminate one of the two states in each of the even and the odd sectors. Eliminating $f_{e-}$ and $f_{o-}$ and keeping $f_{e_{+}}$and $f_{o_{+}}$(this choice is arbitrary, and is equivalent to fixing a phase convention), the completeness sum of Eq. (9.24) is altered into the + state projection operator

$$
\begin{aligned}
P_{+}\left(\psi^{\prime}, \psi\right) & \equiv f_{e+}\left(\psi^{\prime}\right) \bar{f}_{e+}(\psi)+f_{o+}\left(\psi^{\prime}\right) i \bar{f}_{o+}(\psi) \\
& =\frac{1}{2}\left[1+\psi_{0} \psi_{1}+\psi_{0}^{\prime} \psi_{1}^{\prime}+\psi_{0}^{\prime} \psi_{1}^{\prime} \psi_{0} \psi_{1}+i\left(\psi_{0}^{\prime} \psi_{0}+\psi_{1}^{\prime} \psi_{1}\right)-\psi_{0}^{\prime} \psi_{1}+\psi_{1}^{\prime} \psi_{0}\right]
\end{aligned}
$$

Now writing

$$
\psi=\psi_{0}+i \psi_{1}, \quad \psi^{\prime}=\psi_{0}^{\prime}+i \psi_{1}^{\prime}, \quad \bar{\psi}=\psi_{0}-i \psi_{1}, \quad \bar{\psi}^{\prime}=\psi_{0}^{\prime}-i \psi_{1}^{\prime},
$$

a short calculation shows that

$$
\begin{aligned}
\Delta \widetilde{S^{\prime}} & \equiv \frac{i}{2}\left[-\bar{\psi}^{\prime}\left(\psi^{\prime}-\psi\right)+\left(\bar{\psi}^{\prime}-\bar{\psi}\right) \psi\right] \\
& =\psi_{0}^{\prime} \psi_{1}^{\prime}+\psi_{0} \psi_{1}+i\left(\psi_{0}^{\prime} \psi_{0}+\psi_{1}^{\prime} \psi_{1}\right)-\psi_{0}^{\prime} \psi_{1}+\psi_{1}^{\prime} \psi_{0}, \\
\frac{1}{2}\left(\Delta \widetilde{S^{\prime}}\right)^{2} & =\psi_{0}^{\prime} \psi_{1}^{\prime} \psi_{0} \psi_{1},
\end{aligned}
$$

and so Eq. (9.29) can be written as

$$
P_{+}\left(\psi^{\prime}, \psi\right)=\frac{1}{2} e^{4 \bar{S}^{\prime}}
$$

Hence the + state projection operator is equal to the exponential of the discrete action constructed from the symmetrized first order Lagrangian

$$
L=\frac{\partial \bar{\psi}}{\partial t} \psi-\bar{\psi} \frac{\partial \psi}{\partial t}
$$

We have thus recovered the standard result that fermion path integrals constructed using a first order fermion wave equation have a definite, rather than an indefinite, Hilbert space structure, with no zero norm states. ${ }^{10}$ In writing Eq. (9.31)

${ }^{10}$ Usually this result is obtained by using coherent states [14], but since complex coherent states do not appear to have a quaternionic analog, we have followed an alternative route which extends in a natural way to quaternions 
we have actually abused the tilde notation, since although $L$ of Eq. (9.33) is selfadjoint, $L=\bar{L}$, the discrete version of $\Delta \tilde{S}=i \Delta t L$ given in Eq. (9.31) is not anti-selfadjoint. We can get an anti-self-adjoint discrete action $\Delta \tilde{S}$ by adding a term which is higher order in $\Delta t$, and hence vanishes in the continuum limit,

$$
\begin{aligned}
\Delta \widetilde{S} & =\frac{i}{2}\left[-\bar{\psi}^{\prime}\left(\psi^{\prime}-\psi\right)+\left(\bar{\psi}^{\prime}-\bar{\psi}\right) \psi\right]=\Delta \tilde{S}^{\prime}+\frac{i}{2}\left(\bar{\psi}^{\prime}-\bar{\psi}\right)\left(\psi^{\prime}-\psi\right) \\
& =\Delta \tilde{S}^{\prime}-\left(\psi^{\prime}-\psi\right)_{0}\left(\psi^{\prime}-\psi\right)_{1} .
\end{aligned}
$$

Exponentiating $\Delta \widetilde{S}$ and using Eqs. (9.32) and (9.24), we find

$$
\begin{aligned}
e^{\Delta \tilde{S}} & =2 P_{+}\left(\psi^{\prime}, \psi\right)\left[1-\delta\left(\psi^{\prime}-\psi\right)\right]=2 P_{+}\left(\psi^{\prime}, \psi\right)-2 P_{+}(\psi, \psi) \delta\left(\psi^{\prime}-\psi\right) \\
& =2 P_{+}\left(\psi^{\prime}, \psi\right)-\left[P_{+}\left(\psi^{\prime}, \psi\right)+P_{-}\left(\psi^{\prime}, \psi\right)\right]=P_{+}\left(\psi^{\prime}, \psi\right)-P_{-}\left(\psi^{\prime}, \psi\right) .
\end{aligned}
$$

Although Eq. (9.35) does not annihilate the-states, it also does not mix the + and - states, and so an alternative to using $\frac{1}{2} e^{\Delta \tilde{S}^{\prime}}$ in the path integral is to use $e^{\Delta \tilde{S}}$, together with the subsidiary condition of requiring the - components of the wave function to vanish on any one time slice. ${ }^{11}$

Having completed this somewhat lengthy analysis of the complex case, we turn next to the fermionic sector in quaternionic field theory. We choose a 16 state orthogonal basis as follows,

$$
\begin{array}{ll}
f_{e 0 \pm}(\psi)=\frac{1}{\sqrt{2}}\left(1 \pm \psi_{0} \psi_{1} \psi_{2} \psi_{3}\right), & f_{o 0 \pm}(\psi)=\frac{1}{\sqrt{2}}\left(\psi_{0} \mp e_{3} \psi_{1} \psi_{2} \psi_{3}\right) \\
f_{e 1 \pm}(\psi)=\frac{1}{\sqrt{2}}\left(\psi_{0} \psi_{1} \pm \psi_{2} \psi_{3}\right), & f_{o 1 \pm}(\psi)=\frac{1}{\sqrt{2}}\left(\psi_{1} \pm e_{3} \psi_{0} \psi_{2} \psi_{3}\right) \\
f_{e 2 \pm}(\psi)=\frac{1}{\sqrt{2}}\left(\psi_{0} \psi_{2} \pm \psi_{3} \psi_{1}\right), & f_{o 2 \pm}(\psi)=\frac{1}{\sqrt{2}}\left(\psi_{2} \pm e_{3} \psi_{0} \psi_{3} \psi_{1}\right) \\
f_{e 3 \pm}(\psi)=\frac{1}{\sqrt{2}}\left(\psi_{0} \psi_{3} \pm \psi_{1} \psi_{2}\right), & f_{o 3 \pm}(\psi)=\frac{1}{\sqrt{2}}\left(\psi_{3} \pm e_{3} \psi_{0} \psi_{1} \psi_{2}\right)
\end{array}
$$

With respect to the inner product of Eq. (9.1), these have the norms

$$
\left(f_{e a \pm}, f_{e a \pm}\right)= \pm 1, \quad a=0,1,2,3, \quad\left(f_{o a \pm}, f_{o a \pm}\right)=\mp e_{3}, \quad a=0,1,2,3 .
$$

The completeness relation of Eq. (9.15) thus becomes

$$
\begin{aligned}
\delta\left(\psi^{\prime}-\psi\right)= & \left(\psi^{\prime}-\psi\right)_{0}\left(\psi^{\prime}-\psi\right)_{1}\left(\psi^{\prime}-\psi\right)_{2}\left(\psi^{\prime}-\psi\right)_{3} \\
= & \sum_{a=0}^{3}\left[f_{e a+}\left(\psi^{\prime}\right) \bar{f}_{e a+}(\psi)-f_{e a-}\left(\psi^{\prime}\right) \bar{f}_{e a-}(\psi)\right. \\
& \left.+f_{o a+}\left(\psi^{\prime}\right) e_{3} \bar{f}_{o a+}(\psi)-f_{o a-}\left(\psi^{\prime}\right) e_{3} \bar{f}_{o a-}(\psi)\right],
\end{aligned}
$$

\footnotetext{
${ }^{11}$ If one uses $\frac{1}{2} e^{4 \tilde{S}^{\prime}}$, the Hamiltonian is written in the standard form $H_{00}+i H_{01} \psi+\bar{\psi}^{\prime} H_{10}$ $+i \bar{\psi}^{\prime}\left(H_{11}-H_{00}\right) \psi$, while to use $e^{\Delta \tilde{S}}$, one must write the Hamiltonian as $H_{00}+i H_{01} \frac{1}{2}\left(\psi^{\prime}+\psi\right)$ $+\bar{\psi}^{\prime} H_{10}+i \bar{\psi}^{\prime}\left(H_{11}-H_{00}\right) \frac{1}{2}\left(\psi^{\prime}+\psi\right)$, which differs from the standard form by terms of order $\Delta t$
} 
which is readily verified by substituting Eq. (9.36). Next, we compute the action on the basis states of the anti-self-adjoint Hamiltonian [cf. Eq. (4.23)]

$$
\begin{aligned}
\tilde{H}= & \frac{e_{1}}{3}\left(\frac{\partial}{\partial \psi_{2}} \frac{\partial}{\partial \psi_{3}}-\frac{\partial}{\partial \psi_{0}} \frac{\partial}{\partial \psi_{1}}\right) \\
& +\frac{e_{2}}{3}\left(\frac{\partial}{\partial \psi_{3}} \frac{\partial}{\partial \psi_{1}}-\frac{\partial}{\partial \psi_{0}} \frac{\partial}{\partial \psi_{2}}\right)+\frac{e_{3}}{3}\left(\frac{\partial}{\partial \psi_{1}} \frac{\partial}{\partial \psi_{2}}-\frac{\partial}{\partial \psi_{0}} \frac{\partial}{\partial \psi_{3}}\right) .
\end{aligned}
$$

In the even sector we find

$$
\begin{aligned}
& \tilde{H} f_{e 0 \pm}=\mp \frac{1}{3}\left(e_{1} f_{e 1-}+e_{2} f_{e 2-}+e_{3} f_{e 3-}\right), \\
& \tilde{H} f_{e a+}=0, \quad a=1,2,3, \\
& \tilde{H} f_{e a-}=\frac{1}{3} e_{a}\left(f_{e 0+}+f_{e 0-}\right), \quad a=1,2,3 .
\end{aligned}
$$

Hence there is no way of choosing a subset of four non-zero norm states in the even sector which propagate without mixing with states with the opposite sign norm. (The best we can do with a subsidiary condition in the even sector is to restrict to a basis of three positive norm states $f_{e a+}$, and one zero norm state $f_{e 0+}+f_{e 0-}$, which are all annihilated by $\tilde{H}$, and hence propagate without mixing.) In the odd sector we find

$$
\begin{aligned}
& \tilde{H} f_{o 0 \pm}=\mp \frac{1}{6}\left[e_{2}\left(f_{o 1+}+f_{o 1-}\right)-e_{1}\left(f_{o 2+}+f_{o 2-}\right)+f_{o 3+}+f_{o 3-}\right], \\
& \tilde{H} f_{o 1 \pm}= \pm \frac{1}{6}\left[e_{1}\left(f_{o 3+}+f_{o 3-}\right)+e_{2}\left(f_{o 0+}+f_{o 0-}\right)+f_{o 2+}+f_{o 2-}\right], \\
& \tilde{H} f_{o 2 \pm}= \pm \frac{1}{6}\left[e_{2}\left(f_{o 3+}+f_{o 3-}\right)-e_{1}\left(f_{o 0+}+f_{o 0-}\right)-\left(f_{o 1+}+f_{o 1-}\right)\right], \\
& \tilde{H} f_{o 3 \pm}=\mp \frac{1}{6}\left[e_{2}\left(f_{o 2+}+f_{o 2-}\right)+e_{1}\left(f_{o 1+}+f_{o 1-}\right)-\left(f_{o 0+}+f_{o 0-}\right)\right] .
\end{aligned}
$$

We conclude from Eqs. (9.40)-(9.41) that in the quaternionic case, just as in the complex case, the use of a second order fermionic wave operator is necessarily associated with an indefinite metric state structure.

We finally address the question of whether a quaternionic field theory can be formulated using a first order wave operator for the fermions. We consider only the free field case with no potentials, take the bosonic kinetic term to have the form of Eq. (3.5), and examine the term of order $(\Delta t)^{0}$ in Eq. (4.2) for the time development of the wave function $\Psi$. A first order formalism will work for an arbitrary number of degrees of freedom only if, when one bosonic and one fermionic degree of freedom are integrated out, the sum $\mathfrak{R}$ of quaternion-imaginary kinetic Lagrangians for the remaining degrees of freedom re-exponentiates. That is, we must have

$$
e^{\Re} \Psi_{e^{+}}\left(\phi^{\prime}, \psi^{\prime}\right)=\int d \psi e^{\Delta S\left(\psi^{\prime}, \psi\right)} \frac{1}{C} L_{1}\left(\Delta \widetilde{S}\left(\psi^{\prime}, \psi\right)+\Re\right) \Psi_{e^{+}}\left(\phi^{\prime}, \psi\right),
$$

where $\Psi_{e+}$ lies in a 4-state definite-norm subspace of the even part of the fermionic state space, and where we have allowed for the possible presence of a quaternionreal action term of higher order in $\Delta t$ [cf. Eq. (9.34)]

$$
\Delta S\left(\psi^{\prime}, \psi\right)=B\left(\bar{\psi}^{\prime}-\bar{\psi}\right)\left(\psi^{\prime}-\psi\right)
$$


in addition to the quaternion-imaginary first-order action.

$$
\Delta \tilde{S}\left(\psi^{\prime}, \psi\right)=-\frac{1}{2} A\left[\left(\bar{\psi}^{\prime}-\bar{\psi}\right) e_{3} \psi^{\prime}-\bar{\psi}^{\prime} e_{3}\left(\psi^{\prime}-\psi\right)\right] .
$$

It is highly implausible that Eq. (9.42) can be satisfied with any choice of the real constants $A, B, C$ for general quaternion-imaginary $\mathfrak{R}$. A necessary (but not sufficient) condition is that Eq. (9.42) be satisfied for $\mathfrak{R}=0$, which requires

$$
\left.P=e^{\Delta S\left(\psi^{\prime}, \psi\right)} \frac{1}{C} L_{1}\left(\Delta \widetilde{S(} \psi^{\prime}, \psi\right)\right)
$$

to act as a unit projection operator in some 4-state definite-norm subspace of the even part of the fermionic state space. By a tedious calculation (see appendix), we have proved that this is not possible in the case $B=0$. When $B$ is non-zero, there is one additional parameter, but symmetries present when $B=0$ are broken, and so further independent conditions on the parameters $A, B, C$ must be satisfied in order for $P$ to have the required projection property. It is likely that the impossibility proof extends to this case also, but because of the algebraic complexity involved we have not attempted to carry it through.

\section{Open Issues and Future Directions}

We conclude by discussing in this section a number of specific open issues connected with the calculations described above, and then sketch directions which we intend to pursue for the future development of quaternionic field theory. Among the open issues raised by our analysis are:

1. What is the deeper reason for the simplification of quaternionic Gaussian integrals when the numbers of bosonic and fermionic degrees of freedom are equal? One would like to understand why the bosonic recursion relation of Eq. (2.30) and the fermionic recursion relation of Eq. (2.32) are effectively inverse to one another.

2. Is there a natural way of rewriting the representation of $\delta\left(\phi^{\prime}-\phi, \psi^{\prime}-\psi\right)$ obtained from Eq. (2.42) in spectral form,

$$
\begin{gathered}
\delta\left(\phi^{\prime}-\phi, \psi^{\prime}-\psi\right)=\left(\prod_{i=1}^{M} \int d u^{i} d \xi^{i}\right)(2 \pi)^{-4 M} e^{\Phi^{\prime}-\Phi}=(?) \sum_{l} f_{l}\left(\phi^{\prime}, \psi^{\prime}\right) \bar{f}_{l}(\phi, \psi), \\
\Phi^{\prime}=\Phi\left(\phi \rightarrow \phi^{\prime}, \psi \rightarrow \psi^{\prime}\right) .
\end{gathered}
$$

One cannot just write $\exp \left(\Phi^{\prime}-\Phi\right)=\exp \Phi^{\prime} \overline{\exp \Phi}$ because the quaternion units do not commute.

3. Can the derivation of the quaternionic functional integral given in Sect. 5 be simplified? We note here the following curious fact. Suppose we try to follow the exact complex derivation of Eqs. (5.10)-(5.17), and make the (unjustified) approximation, denoted below by $\sim$, of neglecting the non-commutativity of the quaternion units by replacing quaternion unit products $e_{a} e_{b}$ and $e_{b} e_{a}$ by $-\delta_{a b}$. We 
then get instead of Eq. (5.31),

$$
\left(\prod_{i=1}^{M} \int d u^{i} d \xi^{i}\right)(2 \pi)^{-4 M} e^{\Phi-\Delta t\left[\tilde{H}_{\mathrm{kin}}, \Phi\right]},
$$

with

$$
-\Delta t\left[\tilde{H}_{\mathrm{kin}}, \Phi\right]=\Delta t\left[h_{a} e_{a}, \Phi_{b} e_{b}\right] \sim-\delta_{a b} \Delta t\left[h_{a}, \Phi_{b}\right]=-\Delta t \kappa .
$$

Substituting Eq. (10.3) back into Eq. (10.2) and commuting derivatives through to the right (where they vanish) we get

$$
e^{\Phi-\Delta t \kappa} \sim e^{\Phi-\frac{1}{2}[\Phi,-\Delta t \kappa]}=e^{\Phi-\frac{1}{2} \Delta t k}
$$

which is the correct answer! Is this "derivation" an accident, or can a way be found to justify it?

4. The proof of the absence of a first order formalism in quaternionic field theory, started at the end of Sect. 9, needs to be completed.

5. We have shown in Sect. 7 that certain features of complex field theory break down in the quaternionic case. There are many other aspects of the complex formalism, such as the canonical formalism, coherent states, and the Euclidean continuation, for which no apparent quaternionic analog exists. Obviously, not being able to find a construction is not the same as proving none exists; some definitive results are needed here.

6. As discussed briefly at the end of Sect. 4, can one obtain satisfactory quaternionic field theories in the limit $|M-N| \rightarrow \infty$ ?

Some major directions which we intend to pursue in the future, and related questions are:

I. A spatial manifold structure must be included, by reinterpreting the index $i$ labelling degrees of freedom as a spatial coordinate label. One can then gauge the quaternionic unit $e_{3}$ in the bosonic kinetic action, thus realizing the "General $Q$ Covariance" of Finkelstein et al. [15]. The functional integral formalism of Sects. 3-6 will have to be extended to constrained systems and Feynman rules developed in a way which bypasses the canonical formalism; very likely, this can be done by the heuristic method of dividing the functional measure by the infinite gauge orbit volume. The quaternionic field theory analogs of the BRS transformations, Poincaré invariance, graded Poincaré invariance (Poincaré supersymmetry), the TCP theorem, and the spin-statistics connection will all have to be worked out, as well as the renormalization group structure of quaternionic field theory.

II. The fermionic structure must be studied in greater detail. The fermions in the quaternionic field theories we have constructed obey a second-order wave equation. This has two possible advantages: (i) The normalization constant in the functional integral is just a phase space factor which is independent of $\Delta t$, suggesting that perhaps functional integrals with complete boson-fermion symmetry may be mathematically better-behaved objects than functional integrals without such symmetry. (ii) Theories with second order fermions may be manifestly gauge-anomaly free, since they may behave in this regard as do purely bosonic theories [16]. In the supersymmetric case, they may be free of conformal anomalies as well. This is clearly an important question for investigation. 
However, second order fermions have obvious problems: they involve an indefinite metric state space, and they do not correspond to observed fermionic matter. Methods must be developed to get first order fermions from second order, either by gauging away the indefinite metric states [17], or by symmetry breaking which turns the indefinite metric states into heavy, unstable degrees of freedom which do not appear in the asymptotic spectrum [18]. An interesting possibility [19] is that the appearance of chiral fermions in the electroweak interaction, and the fact that these fermions obey a first-order rather than a second-order dynamics, are related.

III. Symmetry breaking mechanisms must be studied, particularly ones which break quaternionic field theory down to complex field theory. Since a commutative quaternionic tensor product does not exist [4,20], asymptotic states and an $S$-matrix can probably only be constructed for a complex substructure of the quaternionic state space, and so symmetry breaking is essential to make contact with observed physics.

IV. When I, II, and III are understood, particle physics model-building may be possible. Some interesting questions here are the following: (i) Can the richer structure of quaternionic field theory lead to the dynamical generation of some of the Lie group structures of the standard model, such as the SU(3) color ${ }^{12}$ or flavor symmetries? Can the breaking of quaternionic field theory down to complex field theory help resolve the hierarchy problem? Do quaternionic field theories offer new mechanisms for solving the cosmological constant problem? (ii) In quaternionic field theory, $e_{3}$ and $-e_{3}$ are related by a unitary transformation which is continuously connected to the identity, whereas in complex field theory, $i$ and $-i$ are related by the anti-unitary operation of $T$ inversion. Can the observed small $T$ violation in particle physics be interpreted as an artifact of the breaking of an underlying quaternionic field theory down to a complex one?

\section{Appendix: Nonexistence of First Order Formalism $(B=0$ Case)}

We consider here the $\Re=0$ case of Eq. (9.42), specialized to $B=0$. Changing variables according to $\psi^{\prime}=\xi, \psi=\xi+\zeta$, and suppressing the boson variable $\phi^{\prime}$, Eq. (9.42) becomes

$$
\begin{gathered}
\Psi_{e+}(\xi)=\int d \zeta \frac{1}{C} L_{1}(\Delta \widetilde{S}[\xi, \zeta]) \Psi_{e+}(\xi+\zeta) \\
\Delta \widetilde{S}[\xi, \zeta]= \\
+\frac{1}{2} A\left(\bar{\zeta} e_{3} \xi-\bar{\xi} e_{3} \zeta\right)=A\left[e_{1}\left(\zeta_{3} \xi_{1}+\zeta_{1} \xi_{3}-\zeta_{2} \xi_{0}-\zeta_{0} \xi_{2}\right)\right. \\
\left.+e_{2}\left(\zeta_{0} \xi_{1}+\zeta_{1} \xi_{0}+\zeta_{2} \xi_{3}+\zeta_{3} \xi_{2}\right)+e_{3}\left(\zeta_{0} \xi_{0}+\zeta_{3} \xi_{3}-\zeta_{1} \xi_{1}-\zeta_{2} \xi_{2}\right)\right]
\end{gathered}
$$

Substituting powers of $\Delta \tilde{S}$ into the expansion

$$
L_{1}(\mathfrak{I})=-1+\frac{1}{3} \mathfrak{J}+\frac{1}{6} \mathfrak{J}^{2}+\frac{1}{10} \mathfrak{J}^{3}+\frac{1}{40} \mathfrak{J}^{4}
$$

\footnotetext{
${ }^{12}$ For an earlier version of these speculations see [21]; the equations of this reference involve complexified quaternions (i.e., use $1, e_{1}, e_{2}, e_{3}$, and $i$ ), and hence do not in fact constitute a quaternionic field theory. I wish to thank C. N. Yang for pointing this out to me
} 
we find after considerable algebra

$$
\begin{aligned}
P(\xi, \zeta) \equiv & \frac{1}{C} L_{1}(\Delta \tilde{S}[\xi, \zeta])=-\frac{1}{C}+\frac{A}{3 C}\left\{e_{1}\left(\zeta_{3} \xi_{1}+\zeta_{1} \xi_{3}-\zeta_{2} \xi_{0}-\zeta_{0} \xi_{2}\right)\right. \\
& \left.+e_{2}\left(\zeta_{0} \xi_{1}+\zeta_{1} \xi_{0}+\zeta_{2} \xi_{3}+\zeta_{3} \xi_{2}\right)+e_{3}\left(\zeta_{0} \xi_{0}+\zeta_{3} \xi_{3}-\zeta_{1} \xi_{1}-\zeta_{2} \xi_{2}\right)\right\} \\
& -\frac{A^{2}}{3 C}\left\{2 \zeta_{0} \zeta_{1} \xi_{0} \xi_{1}+2 \zeta_{0} \zeta_{2} \xi_{0} \xi_{2}+2 \zeta_{2} \zeta_{3} \xi_{2} \xi_{3}+2 \zeta_{3} \zeta_{1} \xi_{3} \xi_{1}\right. \\
& -2 \zeta_{0} \zeta_{3} \xi_{1} \xi_{2}-2 \zeta_{1} \zeta_{2} \xi_{0} \xi_{3}+\zeta_{0} \zeta_{1} \xi_{2} \xi_{3}+\zeta_{0} \zeta_{2} \xi_{3} \xi_{1} \\
& \left.+\zeta_{2} \zeta_{3} \xi_{0} \xi_{1}+\zeta_{3} \zeta_{1} \xi_{0} \xi_{2}-\zeta_{0} \zeta_{3} \xi_{0} \xi_{3}-\zeta_{1} \zeta_{2} \xi_{1} \xi_{2}\right\} \\
& +\frac{A^{3}}{C}\left\{e _ { 1 } \left[-\zeta_{1} \zeta_{2} \zeta_{3} \xi_{0} \xi_{1} \xi_{3}+\zeta_{0} \zeta_{2} \zeta_{3} \xi_{0} \xi_{1} \xi_{2}\right.\right. \\
& \left.-\zeta_{0} \zeta_{1} \zeta_{3} \xi_{1} \xi_{2} \xi_{3}+\zeta_{0} \zeta_{1} \zeta_{2} \xi_{0} \xi_{2} \xi_{3}\right] \\
& +e_{2}\left[-\zeta_{1} \zeta_{2} \zeta_{3} \xi_{0} \xi_{2} \xi_{3}-\zeta_{0} \zeta_{2} \zeta_{3} \xi_{1} \xi_{2} \xi_{3}\right. \\
& \left.-\zeta_{0} \zeta_{1} \zeta_{3} \xi_{0} \xi_{1} \xi_{2}-\zeta_{0} \zeta_{1} \zeta_{2} \xi_{0} \xi_{1} \xi_{3}\right] \\
& +e_{3}\left[\zeta_{1} \zeta_{2} \zeta_{3} \xi_{1} \xi_{2} \xi_{3}-\zeta_{0} \zeta_{2} \zeta_{3} \xi_{0} \xi_{2} \xi_{3}\right. \\
& \left.\left.-\zeta_{0} \zeta_{1} \zeta_{3} \xi_{0} \xi_{1} \xi_{3}+\zeta_{0} \zeta_{1} \zeta_{2} \xi_{0} \xi_{1} \xi_{2}\right]\right\}+\frac{3 A^{4}}{C} \zeta_{0} \zeta_{1} \zeta_{2} \zeta_{3} \xi_{0} \xi_{1} \xi_{2} \xi_{3} . \text { (A }
\end{aligned}
$$

It is convenient to work with a monomial basis in the even sector, labeled as follows

$$
\begin{array}{ll}
g_{0}=1, & h_{0}=\psi_{0} \psi_{1} \psi_{2} \psi_{3}, \\
g_{1}=\psi_{0} \psi_{1}, & h_{1}=\psi_{2} \psi_{3}, \\
g_{2}=\psi_{0} \psi_{2}, & h_{2}=\psi_{3} \psi_{1}, \\
g_{3}=\psi_{0} \psi_{3}, & h_{3}=\psi_{1} \psi_{2} .
\end{array}
$$

Computation of the Grassmann integrals in Eq. (A.1) then gives

$$
P g_{0} \equiv \int d \zeta P(\xi, \zeta) g_{0}(\xi+\zeta)=\frac{3 A^{4}}{C} h_{0}, \quad P h_{0}=-\frac{1}{c} g_{0}
$$

and

$$
\begin{array}{ll}
P\left(g_{1}+h_{1}\right)=-\frac{A^{2}}{C}\left(g_{1}+h_{1}\right), & P\left(g_{1}-h_{1}\right)=\frac{A^{2}}{3 C}\left(g_{1}-h_{1}\right), \\
P\left(g_{2}+h_{2}\right)=-\frac{A^{2}}{C}\left(g_{2}+h_{2}\right), & P\left(g_{2}-h_{2}\right)=\frac{A^{2}}{3 C}\left(g_{2}-h_{2}\right), \\
P\left(g_{3}+h_{3}\right)=+\frac{A^{2}}{C}\left(g_{3}+h_{3}\right), & P\left(g_{3}-h_{3}\right)=\frac{A^{2}}{3 C}\left(g_{3}-h_{3}\right) .
\end{array}
$$

Equations (A.5b) imply that we can only get three definite-norm $P$ eigenstates with $P=1$ from $g_{1,2,3}, h_{1,2,3}$ by taking

$$
\frac{A^{2}}{3 C}=1 \Rightarrow P\left(g_{a}-h_{a}\right)=\left(g_{a}-h_{a}\right), \quad a=1,2,3 \text {. }
$$


However, Eq. (A.5a) then becomes

$$
\begin{gathered}
P g_{0}=27 C h_{0}, \\
P h_{0}=-C^{-1} g_{0} \Rightarrow P\left[h_{0} \pm\left(e_{3} / \sqrt{27} C\right) g_{0}\right]= \pm \sqrt{27} e_{3}\left[h_{0} \pm\left(e_{3} / \sqrt{27} C\right) g_{0}\right],
\end{gathered}
$$

yielding two zero-norm eigenstates with eigenvalues $\pm \sqrt{27} e_{3}$. Hence we cannot find four definite-norm linear combinations of the monomials of Eq. (A.4) on which $P$ is a unit projection, for any values of the real constants $A$ and $C$.

Acknowledgements. I wish to thank J. S. Bell, L. C. Biedenharn, J. D. Bjorken, F. J. Dyson, D. Finkelstein, L. P. Horwitz, R. Langlands, A. McIntosh, M. Mueller, B. Ovrut, N. Seiberg, D. Speiser, A. Strominger, E.P. Wigner, and C. N. Yang for conversations or correspondence relating to issues discussed in this paper. This work was supported by the U.S. Department of Energy Grant No. DE-AC02-76ERO2220.

\section{References}

1. Birkhoff, G., von Neumann, J.: Ann. Math. 37, 823 (1936)

Finkelstein, D., Jauch, M., Schiminovich, S., Speiser, D.: Foundations of quaternion quantum mechanics. J. Math. Phys. 3, 207 (1962)

2. Günaydin, M., Gürsey, F.: An octonionic representation of the Poincaré group. Lett. Nuovo Cimento 6, 401 (1973); Quark statistics and octonions. Phys. Rev. D9, 3387 (1974); Quark structure and octonions. J. Math. Phys. 14, 1651 (1973)

Gürsey, F.: Some algebraic structures in particle theory. Günaydin, M.: Moufang plane and octonionic quantum mechanics. Both in: Proceedings of the second Johns Hopkins workshop. Domokos, G., Kövesi-Domokos, S. (eds.). Baltimore: Johns Hopkins 1978 Günaydin, M., Piron, C., Ruegg, H.: Moufang plane and octonionic quantum mechanics. Commun. Math. Phys. 61, 69 (1978)

3. Stueckelberg, E.C.G.: Théorie des quanta dans l'espace de Hilbert réel I, II, III et IV. Helv. Phys. Acta 33, 727 (1960); 34, 621, 675 (1961), 35, 673 (1962)

4. See especially Finkelstein, D., Jauch, J.M., Schiminovich, S., Speiser, D.: [1] above and Principle of general $Q$ covariance. J. Math. Phys. 4, 788 (1963)

Finkelstein, D., Jauch, J.M., Speiser, D.: Quaternionic representations of compact groups. J. Math. Phys. 4, 136 (1963) and: Notes on quaternion quantum mechanics. In: Logico-algebraic approach to quantum mechanics II. Hooker, C..(ed.). Dordrecht: Reidel 1959

Emch, G.: Mécanique quantique quaternionienne et Relativité restreinte I. Helv. Phys. Acta 36, 739, 770 (1963)

Dyson, F.J.: The threefold way. Algebraic structure of symmetry groups and ensembles in quantum mechanics. J. Math. Phys. 3, 1199 (1962), Correlations between eigenvalues of a random matrix. Commun. Math. Phys. 19, 235 (1970), and Quaternion determinants. Helv. Phys. Acta 45, 289 (1972)

Mehta, M.L.: Elements of matrix theory. Delhi: Hindustan 1977

Horwitz, L.P., Biedenharn, L.C.: Quaternion quantum mechanics: second quantization and gauge fields. Ann. Phys. (New York) 157, 432 (1984)

5. Adler, S.L.: Quaternionic quantum field theory. Phys. Rev. Letts. 55, 783 (1985)

6. Adler, S.L.: Quaternionic Gaussian multiple integrals. In: Quantum field theory and quantum statistics: Essays in honor of the $60^{\text {th }}$ birthday of E. S. Fradkin. Batalin, I.A., Isham, C.J., Vilkovisky, G.A. (eds.), to be published by Adam Hilger

7. Finkelstein, D., Jauch, J.M., Speiser, D.: Notes on quaternion quantum mechanics. Ref. [4] op. cit., Sect. 4

8. Dirac, P.A.M.: The Lagrangian in quantum mechanics. Phys. Sowjetunion 3, 26 (1933) 
9. Feynman, R.P.: Space-time approach to non-relativistic quantum mechanics. Rev. Mod. Phys. 20, 367 (1948)

10. Finkelstein, D., Jauch, J.M., Schiminovich, S., Speiser, D.: Ref. [1] op. cit., Sect. 5

11. Nelson, E.: Feynman integrals and the Schrödinger equation. J. Math. Phys. 5, 332 (1964)

12. Horwitz, L.P., Biedenharn, L.C.: Ref. [4] op. cit., Eq. (3.15) and Theorem III.2

13. Faddeev, L.D., Slavnov, A.A.: Gauge fields: Introduction to quantum theory. Reading: Benjamin/Cummings 1980, Sect. 3.4

14. Candlin, D.J.: On sums over trajectories for systems with Fermi statistics. Nuovo Cimento 4, 231 (1956)

Berezin, F.A.: The method of second quantization. New York: Academic 1966

For a very brief and clear account, see Halpern, M.B., Jevicki, A., Senjanović, P.: Field theories in terms of particle-string variables: Spin, internal symmetry and arbitrary dimension. Phys. Rev. D 16, 2476 (1977), Appendix

15. Finkelstein, D., Jauch, J.M., Schiminovich, S., Speiser, D.: Ref. [4] op. cit.,

16. Bardeen, W.A.: Regularization of gauge field theories. In: Proceedings of the XVI international conference on high energy physics, Fermilab, Batavia, 1972, Vol. 2, p. 295

17. Schwartz, J.: Introduction to superstrings. In: Proceedings of the April 1984 Spring School on supergravity and supersymmetry, Trieste, Italy, discussion following Eq. (25)

18. Lee, T.D., Wick, G.C.: Negative metric and the unitarity of the $S$-matrix. Nucl. Phys. B 9, 209 (1969)

Tomboulis, E.T.: On unitarity in renormalizable $R_{\mu \nu}^{2}$-quantum gravity. To appear in the Fradkin birthday volume, Ref. [6] op. cit.

19. Feynman, R.P., Gell-Mann, M.: Theory of the Fermi interaction. Phys. Rev. 109, 193 (1958)

20. Brackx, F., Delanghe, R., Sommen, F.: Clifford Analysis. Boston: Pittman 1982, Sect. 4.6

21. Adler, S.L.: Quaternionic chromodynamics as a theory of composite quarks and leptons. Phys. Rev. D 21, 2903 (1980)

Communicated by A. Jaffe

Received October 28, 1985; in revised form November 13, 1985 